Pacific

Journal of

Mathematics

UNIVALENCE OF EQUIVARIANT RIEMANN DOMAINS OVER THE COMPLEXIFICATIONS OF RANK-ONE RIEMANNIAN SYMMETRIC SPACES

LAURA GEATTI AND ANDREA IANNUZZI

Volume $238 \quad$ No. 2

December 2008 


\title{
UNIVALENCE OF EQUIVARIANT RIEMANN DOMAINS OVER THE COMPLEXIFICATIONS OF RANK-ONE RIEMANNIAN SYMMETRIC SPACES
}

\author{
LAURA GEATTI AND ANDREA IANNUZZI
}

\begin{abstract}
Let $G / K$ be a noncompact, rank-one, Riemannian symmetric space, and let $G^{\mathbb{C}}$ be the universal complexification of $G$. We prove that a holomorphically separable, $G$-equivariant Riemann domain over $G^{\mathbb{C}} / K^{\mathbb{C}}$ is necessarily univalent, provided that $G$ is not a covering of $\operatorname{SL}(2, \mathbb{R})$. As a consequence, one obtains a univalence result for holomorphically separable, $G \times K$-equivariant Riemann domains over $G^{\mathbb{C}}$. Here $G \times K$ acts on $G^{\mathbb{C}}$ by left and right translations. The proof of such results involves a detailed study of the $G$-invariant complex geometry of the quotient $G^{\mathbb{C}} / K^{\mathbb{C}}$, including a complete classification of all its Stein $G$-invariant subdomains.
\end{abstract}

\section{Introduction}

Let $Y$ be a domain in a Stein manifold $X$. By a classical result of H. Rossi [1963], the envelope of holomorphy of $Y$ exists and can be realized as a Riemann domain $\hat{p}: \hat{Y} \rightarrow X$. In general it is a difficult problem to explicitly determine $\hat{Y}$ and to establish whether $\hat{p}$ is injective, that is, whether the envelope of holomorphy $\hat{Y}$ is univalent. However, in the presence of a large group of symmetries, some results are known. For instance, let the vector group $G=\left(\mathbb{R}^{n},+\right)$ act on its universal complexification $G^{\mathbb{C}}=\left(\mathbb{C}^{n},+\right)$ by left multiplication. Bochner's tube theorem characterizes the envelope of holomorphy of a $G$-invariant domain $Y$ in $G^{\mathbb{C}}$ as the smallest, convex, $G$-invariant domain in $G^{\mathbb{C}}$ containing $Y$. In particular it shows that such envelope is univalent. An analogous statement holds true for $G$ a compact torus, that is, for envelopes of holomorphy of Reinhardt domains in $\left(\mathbb{C}^{*}\right)^{n}$.

Let $G$ be a connected Lie group, and let $Y$ be a complex $G$-manifold, that is, a complex manifold endowed with a real-analytic action of $G$ by holomorphic transformations. A $G$-equivariant Riemann domain over $G^{\mathbb{C}}$ is by definition a $G$-equivariant local biholomorphism $p: Y \rightarrow G^{\mathbb{C}}$. A motivation for determining conditions under which $p$ is injective in this more general context comes from the theory of globalization of local actions. Namely, given a reduced complex

MSC2000: 32D26, 32Q28, 53C35, 32M05.

Keywords: Riemann domain, semisimple Lie group, symmetric space. 
space endowed with a holomorphic $G$-action, one can consider the induced local $G^{\mathbb{C}}$-action. It turns out that the univalence of $G$-equivariant Riemann domains over $G^{\mathbb{C}}$ is a necessary condition for extending such a local action to a global one; see [Palais 1957; Heinzner and Iannuzzi 1997; Casadio Tarabusi et al. 2000].

For a certain class of groups, including for example the product of a compact and a simply connected nilpotent Lie group, univalence results were obtained for arbitrary holomorphically separable, $G$-equivariant Riemann domains over $G^{\mathbb{C}}$ by Cœuré and Loeb [1986]. Note that since $G^{\mathbb{C}}$ is Stein (see [Heinzner 1993]), holomorphic separability of $Y$ is a necessary condition for $p$ to be injective.

When $G$ is a noncompact, real semisimple Lie group, univalence of holomorphically, separable $G$-equivariant Riemann domains over $G^{\mathbb{C}}$ does not hold in general. For $G=\mathrm{SL}(2, \mathbb{R})$, a Stein counterexample was pointed out to us by K. Oeljeklaus; see Section 8. The image of this Riemann domain in $G^{\mathbb{C}}$ is also invariant under right $K$-translations, and its construction is based on the existence of nontrivial coverings of the $K$-orbits in $G^{\mathbb{C}}$. Here $K$ is a maximal compact subgroup in $G$. Observe also that $\operatorname{SL}(2, \mathbb{C}) / \operatorname{SL}(2, \mathbb{R})$ is simply connected. Thus this example gives a negative answer to the question of whether the simple-connectivity of the quotient $G^{\mathbb{C}} / G$ is a sufficient condition for univalence of $G$-equivariant Riemann domains over $G^{\mathbb{C}}$; see [Cœuré and Loeb 1986].

Let $G$ be a connected, noncompact, real simple Lie group, and let $K$ be a maximal compact subgroup of $G$. The group $G$ is not necessarily embedded in $G^{\mathbb{C}}$, but it is assumed to have finite center. Consider the action of the product group $G \times K$ on $G^{\mathbb{C}}$ by left and right translations. One of the results of this paper is the following theorem, Theorem 8.1.

Theorem. Let $G / K$ be a noncompact, rank-one, Riemannian symmetric space. A holomorphically separable, $G \times K$-equivariant Riemann domain $p: Y \rightarrow G^{\mathbb{C}}$ is univalent, provided that $G$ is not a covering of $\operatorname{SL}(2, \mathbb{R})$.

Note that since $Y$ embeds equivariantly into its envelope of holomorphy (see Section 2), there is no loss of generality in assuming that $Y$ is Stein. Then a result of P. Heinzner [1991] implies that the categorical quotient $Y / / K$ is also Stein. By performing categorical $K$-reduction on both $Y$ and $G^{\mathbb{C}}$, one can associate to $p: Y \rightarrow G^{\mathbb{C}}$ a Stein, $G$-equivariant Riemann domain $q: Y / / K \rightarrow G^{\mathbb{C}} / K^{\mathbb{C}}$. A suitable characterization of the univalence of $q$ (see Proposition 3.1) implies that $p$ is univalent if $q$ is univalent. Then the above theorem is a consequence of the following one, which is the main result of the paper; see Theorem 7.6 and Remark 7.8.

Theorem. Let $G / K$ be a noncompact, rank-one, Riemannian symmetric space. A holomorphically separable, G-equivariant Riemann domain $q: \Sigma \rightarrow G^{\mathbb{C}} / K^{\mathbb{C}}$ is univalent, provided that $G$ is not a covering of $\operatorname{SL}(2, \mathbb{R})$. 
The proof of this theorem is carried out as follows. First we show that, with few exceptions, the map $q$ is injective on every $G$-orbit. For principal $G$-orbits this is done by determining their topology. The result is then extended to the remaining $G$-orbits by a general argument in Section 5. As a consequence there exists a $G$-invariant domain in $\Sigma$ on which $q$ is injective.

Next we show that such domain can be enlarged to the whole $\Sigma$. This is done by successively lifting to $\Sigma$ local slices for principal $G$-orbits in $G^{\mathbb{C}} / K^{\mathbb{C}}$. Since such slices are one-dimensional and $q$ is injective on $G$-orbits, each lifting determines a $G$-invariant domain in $\Sigma$ on which $q$ is injective. The main difficulty is in ensuring monodromy around singular $G$-orbits. For this we combine a detailed description of the $G$-orbit structure of $G^{\mathbb{C}} / K^{\mathbb{C}}$ with the complex-geometric properties of certain non-Stein, $G$-invariant domains in $G^{\mathbb{C}} / K^{\mathbb{C}}$.

By the above univalence result, all Stein, $G$-equivariant Riemann domains over $G^{\mathbb{C}} / K^{\mathbb{C}}$ can be regarded as Stein, invariant domains in $G^{\mathbb{C}} / K^{\mathbb{C}}$. We carry out their classification in Theorem 6.1. These results also provide examples of Kobayashi hyperbolic domains whose envelopes of holomorphy are not Kobayashi hyperbolic; see Example 7.9.

For $G / K$ of arbitrary rank, recent investigations due to several authors have indicated an interplay between the complex geometry of distinguished Stein, $G$ invariant domains in $G^{\mathbb{C}} / K^{\mathbb{C}}$ and the harmonic analysis on the $G$-symmetric spaces contained in $G^{\mathbb{C}} / K^{\mathbb{C}}$; see [Krötz and Stanton 2005; Fels et al. 2006] and references therein. A better understanding of envelopes of holomorphy of $G$-invariant domains in $G^{\mathbb{C}} / K^{\mathbb{C}}$ might give new insights in this context. We hope the present paper to be a first step for further investigations in higher rank.

The paper is organized as follows. In Section 2, we recall some basic notions and results from geometric invariant theory. In Section 3, from a Stein $G \times K$ equivariant Riemann domain $p: Y \rightarrow G^{\mathbb{C}}$ we obtain a Stein, $G$-equivariant, Riemann domain $q: Y / / K \rightarrow G^{\mathbb{C}} / K^{\mathbb{C}}$. We also show that $p$ is univalent if $q$ is univalent. In Section 4, we give a detailed description of the $G$-orbit structure of $G^{\mathbb{C}} / K^{\mathbb{C}}$ when $G / K$ is a noncompact, rank-one, Riemannian symmetric space. We also describe an explicit model for the space $G^{\mathbb{C}} / K^{\mathbb{C}}$ in the cases $G=\operatorname{SO}_{0}(n, 1)$ and $G=\mathrm{SU}(n, 1)$. In Section 5, we show that, with few exceptions, a $G$-equivariant Riemann domain $q: Y \rightarrow G^{\mathbb{C}} / K^{\mathbb{C}}$ is univalent on every $G$-orbit. In Section 6, we carry out a complete classification of Stein, $G$-invariant domains in $G^{\mathbb{C}} / K^{\mathbb{C}}$. When $G=\mathrm{SU}(n, 1)$ some of these domains appear to be new. In Section 7, we prove the univalence result for holomorphically separable, $G$-equivariant Riemann domains over $G^{\mathbb{C}} / K^{\mathbb{C}}$. In Section 8, we obtain the result for holomorphically separable, $G \times K$-equivariant Riemann domains over $G^{\mathbb{C}}$. We also discuss some examples. In the appendix, Section 9, we compute the Levi form of all nonclosed hypersurface $G$-orbits in $G^{\mathbb{C}} / K^{\mathbb{C}}$. The results of this computation are used in Sections 6 and 7. 


\section{Preliminaries}

Let $G$ be a connected, real Lie group. A complex Lie group $G^{\mathbb{C}}$ together with a Lie group homomorphism $\iota: G \rightarrow G^{\mathbb{C}}$ is called a universal complexification of $G$ if, for every Lie group homomorphism $\psi$ from $G$ to a complex Lie group $H$, there exists a holomorphic homomorphism $\psi^{\mathbb{C}}: G^{\mathbb{C}} \rightarrow H$ such that $\psi=\psi^{\mathbb{C}} \circ \iota$. A universal complexification of $G$ always exists and is unique up to biholomorphisms; see [Hochschild 1965].

Assume that $G$ is a connected, real semisimple Lie group. Denote by $\mathfrak{g}$ the Lie algebra of $G$ and by $\mathfrak{g}^{\mathbb{C}}:=\mathfrak{g} \oplus i \mathfrak{g}$ its complexification. Then the universal complexification of $G$ is a complex semisimple Lie group $G^{\mathbb{C}}$ with Lie algebra $\mathfrak{g}^{\mathbb{C}}$. If $G$ is a real form of a simply connected complex semisimple Lie group $G^{\mathbb{C}}$, then its universal complexification is $G^{\mathbb{C}}$. Furthermore, if $\Gamma$ is a central subgroup of $G$, then the universal complexification of the quotient group $G / \Gamma$ is given by $G^{\mathbb{C}} / \Gamma$. Note that every automorphism of $G$ uniquely extends to a holomorphic automorphism of its universal complexification $G^{\mathbb{C}}$.

Let $K$ be a compact Lie group and $X$ a Stein $K$-space, that is, a reduced Stein space with a real-analytic action of $K$ by holomorphic transformations. The categorical quotient $X / / K$ of $X$ is defined by the equivalence relation in which $x \sim y$ if and only if $f(x)=f(y)$ for every $K$-invariant holomorphic function $f$ on $X$. We recall some basic properties of the categorical quotient; see [Heinzner 1991].

Theorem 2.1. Let $K$ be a compact Lie group and $X$ a Stein $K$-space. Then

(i) the categorical quotient $X / / K$ equipped with the algebra $\mathcal{O}(X)^{K}$ of holomorphic $K$-invariant functions on $X$ is a Stein space, and the projection $\pi: X \rightarrow X / / K$ is holomorphic; and

(ii) for every $K$-invariant holomorphic map $\psi$ from $X$ to a complex space $Y$, there exists a unique holomorphic map $\widehat{\psi}: X / / K \rightarrow Y$ making the diagram

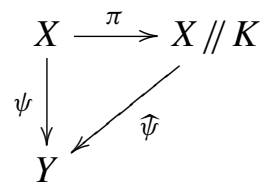

commute.

If the $K$-action on $X$ is the restriction of a $K^{\mathbb{C}}$-action, then the algebras of $K$ invariant and of $K^{\mathbb{C}}$-invariant holomorphic functions on $X$ coincide. In particular, they induce the same equivalence relation on $X$ and $X / / K \cong X / / K^{\mathbb{C}}$. In this case, if all $K^{\mathbb{C}}$-orbits are closed, then $X / / K^{\mathbb{C}}$ coincides with the usual orbit space $X / K^{\mathbb{C}}$; see [Snow 1982, Theorem 3.8]. A $K$-action on a Stein space can always be extended to a $K^{\mathbb{C}}$-action, as shown by the following theorem. 
Theorem 2.2 [Heinzner 1991]. Let $K$ be a compact Lie group and $X$ a Stein $K$ space. Then there exist a Stein $K^{\mathbb{C}}$-space $X^{*}$ and a $K$-equivariant holomorphic embedding $\iota: X \hookrightarrow X^{*}$ with the following properties:

(i) the map $\iota$ is open, and $K^{\mathbb{C}} \cdot \iota(X)=X^{*}$;

(ii) for every $K$-equivariant holomorphic map $\varphi$ from $X$ into a complex $K^{\mathbb{C}}$ space $Z$, there exists a unique $K^{\mathbb{C}}$-equivariant holomorphic map $\varphi^{*}: X^{*} \rightarrow Z$ making the diagram

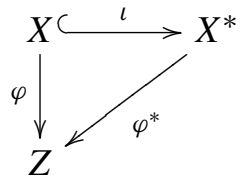

commute;

(iii) the inclusion $X \hookrightarrow X^{*}$ induces an isomorphism between the categorical quotients $X / / K$ and $X^{*} / / K^{\mathbb{C}}$.

Observe that, since $K^{\mathbb{C}} \cdot \iota(X)=X^{*}$, if $X$ is nonsingular, then $X^{*}$ is also nonsingular.

Let $X$ be a complex manifold, and let $G$ be a Lie group. A Riemann domain over $X$ is a complex manifold $Y$ together with a locally biholomorphic map $p$ : $Y \rightarrow X$. If both $X$ and $Y$ are $G$-manifolds and the map $p$ is $G$-equivariant, then we refer to $p: Y \rightarrow X$ as a G-equivariant Riemann domain. If $X$ is Stein and $Y$ is holomorphically separable, then $Y$ embeds as an open domain in its envelope of holomorphy $\widehat{Y}$, and the map $p$ extends to a local biholomorphism $\hat{p}: \widehat{Y} \rightarrow X$; see [Rossi 1963]. Moreover the $G$-action on $Y$ extends to $\widehat{Y}$, and the map $\hat{p}$ is $G$-equivariant, that is, $\hat{p}: \widehat{Y} \rightarrow X$ is a Stein, $G$-equivariant Riemann domain.

A Riemann domain $p: Y \rightarrow X$ is called univalent if the map $p$ is injective. Assume $X$ is Stein and $Y$ is holomorphically separable. If $\hat{p}$ is univalent, then $p$ is also univalent. Aiming at univalence results for holomorphically separable Riemann domains over $G^{\mathbb{C}}$, it is therefore not restrictive to start with Riemann domains that are Stein.

\section{From Riemann domains over $G^{\mathbb{C}}$ to Riemann domains over $G^{\mathbb{C}} / K^{\mathbb{C}}$}

Let $G$ be a connected, noncompact, real semisimple Lie group, let $K \subset G$ be a maximal compact subgroup, and let $G^{\mathbb{C}}$ be the universal complexification of $G$. Let $G \times K$ act on $G^{\mathbb{C}}$ by left and right translations, that is,

$$
(g, k) \cdot z:=g z k^{-1} \quad \text { for }(g, k) \in G \times K \text { and } z \in G^{\mathbb{C}} .
$$

In this section, to every Stein, $G \times K$-equivariant Riemann domain $p: Y \rightarrow G^{\mathbb{C}}$ we associate a Stein, $G$-equivariant Riemann domain $q: \Sigma \rightarrow G^{\mathbb{C}} / K^{\mathbb{C}}$. We also show that the univalence of $q$ implies that of $p$. 
Let $X$ be a Stein $K^{\mathbb{C}}$-manifold and let $p: Y \rightarrow X$ be a Stein, $K$-equivariant Riemann domain. By Theorem 2.2, there exist a Stein $K^{\mathbb{C}}$-manifold $Y^{*}$, a $K$ equivariant holomorphic open embedding $\iota: Y \hookrightarrow Y^{*}$, and a $K^{\mathbb{C}}$-equivariant holomorphic map $p^{*}: Y^{*} \rightarrow X$ such that the diagram

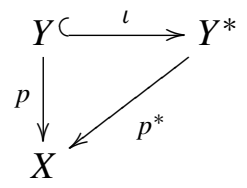

commutes. Since $p$ is locally biholomorphic, $p^{*}$ is $K^{\mathbb{C}}$-equivariant, and $Y^{*}=$ $K^{\mathbb{C}} \cdot Y$, one has that $p^{*}$ is locally biholomorphic as well, that is, it defines a Stein $K^{\mathbb{C}}$-equivariant Riemann domain. By Theorem 2.2, the spaces $Y^{*} / / K^{\mathbb{C}}$ and $Y / / K$ are biholomorphic. Therefore Theorem 2.1 implies there exists a holomorphic map $q: Y / / K \rightarrow X / / K^{\mathbb{C}}$ making the diagram

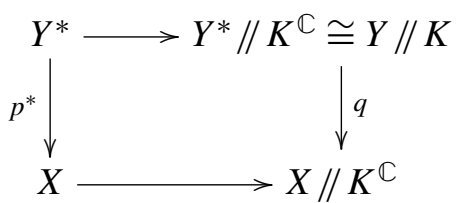

commute. Here the horizontal arrows denote the categorical quotient maps.

Assume that all $K^{\mathbb{C}}$-orbits in $X$ are closed and all $K^{\mathbb{C}}$-isotropies are connected. We claim that all $K^{\mathbb{C}}$-orbits in $Y^{*}$ are closed as well. Suppose by contradiction that there exists a nonclosed orbit $K^{\mathbb{C}} \cdot y$ in $Y^{*}$. Let $K^{\mathbb{C}} \cdot z$ be a lower dimensional orbit in its closure; see [Snow 1982, Proposition 2.3]. Since $p^{*}$ is locally biholomorphic and $K^{\mathbb{C}}$-equivariant, the orbit $K^{\mathbb{C}} \cdot p^{*}(z)$ lies in the closure of $K^{\mathbb{C}} \cdot p^{*}(y)$ and has lower dimension. In particular such orbits are distinct. It follows that the orbit $K^{\mathbb{C}} \cdot p^{*}(y)$ is not closed, contradicting the assumption.

By the above claim, the categorical quotients $X / / K^{\mathbb{C}}$ and $Y^{*} / / K^{\mathbb{C}}$ coincide with the orbit spaces $X / K^{\mathbb{C}}$ and $Y^{*} / K^{\mathbb{C}}$, respectively. If we also assume that the $K^{\mathbb{C}}$-orbits have connected isotropy subgroups, such orbit spaces are nonsingular and the map $q: Y / / K \rightarrow X / K^{\mathbb{C}}$ defines a Stein Riemann domain. We refer to it as the Riemann domain induced by $p: Y \rightarrow X$. Next we prove a general univalence result for Stein, $K$-equivariant Riemann domains.

Proposition 3.1. Let $X$ be a Stein $K^{\mathbb{C}}$-manifold, all of whose $K^{\mathbb{C}}$-orbits are closed and have connected isotropy subgroups. Let $p: Y \rightarrow X$ be a Stein, $K$-equivariant Riemann domain, and let $p^{*}: Y^{*} \rightarrow X$ be its extension to the $K^{\mathbb{C}}$-globalization $Y^{*}$ of $Y$.

(i) The induced Stein, Riemann domain $q: Y / / K \rightarrow X / K^{\mathbb{C}}$ is univalent if and only if $p^{*}: Y^{*} \rightarrow X$ is univalent. 
(ii) If $q: Y / / K \rightarrow X / K^{\mathbb{C}}$ is univalent, then $p: Y \rightarrow X$ is univalent.

Proof. (i) If $p^{*}$ is injective, then it maps distinct $K^{\mathbb{C}}$-orbits in $Y^{*}$ onto distinct $K^{\mathbb{C}}$-orbits in $X$. As we already noticed, since all $K^{\mathbb{C}}$-orbits in $X$ are closed, the categorical quotients $X / / K^{\mathbb{C}}$ and $Y^{*} / / K^{\mathbb{C}}$ coincide with the orbit spaces $X / K^{\mathbb{C}}$ and $Y^{*} / K^{\mathbb{C}}$, respectively. It follows that the induced map $Y^{*} / K^{\mathbb{C}} \rightarrow X / K^{\mathbb{C}}$ is injective. Moreover, by Theorem 2.2, the space $Y / / K$ can be identified with $Y^{*} / / K^{\mathbb{C}}$. As a result the induced Riemann domain $q: Y / / K \rightarrow X / K^{\mathbb{C}}$ is univalent.

Conversely, assume that $q: Y / / K \rightarrow X / K^{\mathbb{C}}$ is univalent, that is, that the map $Y^{*} / K^{\mathbb{C}} \rightarrow X / K^{\mathbb{C}}$ is injective. By assumption, the $K^{\mathbb{C}}$-isotropy subgroups in $X$ are connected; thus $p^{*}$ is injective on every $K^{\mathbb{C}}$-orbit in $Y^{*}$. It follows that $p^{*}: Y^{*} \rightarrow X$ is globally injective. This proves (i); statement (ii) is a direct consequence.

Remark 3.2. In general, under the assumptions of the above proposition, the univalence of $p: Y \rightarrow X$ does not imply that of $q: Y / / K \rightarrow X / K^{\mathbb{C}}$. For instance, let $\mathbb{C}^{*}$ act on $\mathbb{C} \times \mathbb{C}^{*}$ and on $X:=\mathbb{C}^{*} \times \mathbb{C}^{*}$ by multiplication on the second factor. Define $p^{*}: \mathbb{C} \times \mathbb{C}^{*} \rightarrow X$ by $(z, w) \mapsto\left(e^{z}, w\right)$ and consider

$$
Y:=\left\{(z, w) \in \mathbb{C} \times \mathbb{C}^{*}: \operatorname{Im} z<|w|<2 \pi+\operatorname{Im} z\right\} .
$$

Then $Y$ is a Stein $S^{1}$-invariant subdomain of $Y^{*}=\mathbb{C} \times \mathbb{C}^{*}$ and the map $p:=\left.p^{*}\right|_{Y}$ is injective. Nevertheless the induced map $q: Y / / S^{1} \cong \mathbb{C} \rightarrow X / \mathbb{C}^{*} \cong \mathbb{C}^{*}$, given by $z \mapsto e^{z}$, is not injective.

Consider now the case when $X$ is the group $G^{\mathbb{C}}$ endowed with the $G \times K$-action by left and right translations. Let $p: Y \rightarrow G^{\mathbb{C}}$ be a Stein, $G \times K$-equivariant Riemann domain. Note that the actions of $G$ and $K$ commute on $G^{\mathbb{C}}$. Thus they also commute on $Y$, because $p$ is equivariant and locally injective. Since the $K$ action on $G^{\mathbb{C}}$ is the restriction of a $K^{\mathbb{C}}$-action all of whose orbits are closed, the spaces $G^{\mathbb{C}} / / K$ and $G^{\mathbb{C}} / K^{\mathbb{C}}$ are biholomorphic.

By the universality property of the categorical quotient (see Theorem 2.1), the $G$-actions on $Y$ and on $G^{\mathbb{C}}$ induce $G$-actions on $Y / / K$ and on $G^{\mathbb{C}} / K^{\mathbb{C}}$, respectively. Moreover the induced Stein, Riemann domain $q: Y / / K \rightarrow G^{\mathbb{C}} / K^{\mathbb{C}}$ is $G$ equivariant. By applying Proposition 3.1 to this situation, we obtain the following.

Corollary 3.3. Let $p: Y \rightarrow G^{\mathbb{C}}$ be a Stein, $G \times K$-equivariant Riemann domain over $G^{\mathbb{C}}$, and let $q: Y / / K \rightarrow G^{\mathbb{C}} / K^{\mathbb{C}}$ be the induced Stein, $G$-equivariant Riemann domain over $G^{\mathbb{C}} / K^{\mathbb{C}}$. If $q$ is univalent, then $p$ is univalent.

\section{4. $G$-orbit structure of $G^{\mathbb{C}} / K^{\mathbb{C}}$}

Let $G$ be a connected, noncompact, real simple Lie group, let $K \subset G$ be a maximal compact subgroup, and let $G^{\mathbb{C}}$ be the universal complexification of $G$. Assume that $G$ is embedded in $G^{\mathbb{C}}$. The quotient $G / K$ is a Riemannian symmetric space of the 
noncompact type. In this section we obtain a complete description of the $G$-orbit structure of $G^{\mathbb{C}} / K^{\mathbb{C}}$ in the case when $G / K$ has rank one.

We recall some basic facts holding for $G / K$ of arbitrary rank. Denote by $\sigma$ the antiholomorphic involution of $G^{\mathbb{C}}$ relative to $G$ and by $\tau: G^{\mathbb{C}} \rightarrow G^{\mathbb{C}}$ the holomorphic extension of the Cartan involution $\theta$ of $G$ with respect to $K$. Note that the fixed point set of $\tau$ in $G^{\mathbb{C}}$ contains the complexification $K^{\mathbb{C}}$ of $K$. The commuting composition $\sigma \circ \tau=\tau \circ \sigma$ is a Cartan involution of $G^{\mathbb{C}}$. Denote by $U$ the corresponding compact real form. The $U$-orbit of the base point $e K^{\mathbb{C}}$ in $G^{\mathbb{C}} / K^{\mathbb{C}}$ is diffeomorphic to the compact dual symmetric space $U / K$, and is embedded in $G^{\mathbb{C}} / K^{\mathbb{C}}$ transversally to $G / K$.

Remark 4.1. (i) For every triple $\left(G, K, G^{\mathbb{C}}\right)$ as above, the manifold $G^{\mathbb{C}} / K^{\mathbb{C}}$ is simply connected. To see this, denote by $\widetilde{G}^{\mathbb{C}}$ and $\widetilde{U} \subset \widetilde{G}^{\mathbb{C}}$ the universal coverings of $G^{\mathbb{C}}$ and $U$, respectively. Let $\widehat{G}$ be the real form of $\widetilde{G}^{\mathbb{C}}$ relative to the lifting of $\sigma$ to $\widetilde{G}^{\mathbb{C}}$. The group $\widehat{G}$ is connected (see [Steinberg 1968]) and is a finite covering of $G$. Hence $G=\widehat{G} / \Gamma$, where $\Gamma$ is a finite central subgroup of $\widehat{G}$. Similarly $K=$ $\widehat{K} / \Gamma$, where $\widehat{K}$ is a maximal compact subgroup of $\widehat{G}$. One has $G^{\mathbb{C}} \cong \widetilde{G}^{\mathbb{C}} / \Gamma$ (see Section 2) and consequently $U=\widetilde{U} / \Gamma$. As a consequence there are isomorphisms

$$
U / K \cong \widetilde{U} / \Gamma / \widehat{K} / \Gamma \cong \widetilde{U} / \widehat{K}
$$

Since $\widehat{K}$ is connected, the quotient $\widetilde{U} / \widehat{K}$ is simply connected. Moreover $U / K$ is a topological retract of $G^{\mathbb{C}} / K^{\mathbb{C}}$. Hence the claim follows.

(ii) From different triples $\left(G, K, G^{\mathbb{C}}\right)$ as above associated with the same Riemannian symmetric space, one obtains the same complexification $G^{\mathbb{C}} / K^{\mathbb{C}}$. Indeed the map $\widetilde{G}^{\mathbb{C}} / \widehat{K}^{\mathbb{C}} \rightarrow G^{\mathbb{C}} / K^{\mathbb{C}}$, given by $g \widehat{K}^{\mathbb{C}} \mapsto g \Gamma K^{\mathbb{C}}$, defines a biholomorphism. Moreover the center of $G$ acts trivially on $G^{\mathbb{C}} / K^{\mathbb{C}}$. As a consequence, different triples $\left(G, K, G^{\mathbb{C}}\right)$ yield the same $G$-orbit structure of $G^{\mathbb{C}} / K^{\mathbb{C}}$ and $G$-equivariantly diffeomorphic orbits.

Closed $G$-orbits of maximal dimension form an open dense subset of $G^{\mathbb{C}} / K^{\mathbb{C}}$ and come in a finite number of orbit types. We refer to them as principal $G$-orbits. They have real codimension equal to the rank of $G / K$. Singular orbits are closed $G$-orbits that are not principal.

The $G$-orbit structure of $G^{\mathbb{C}} / K^{\mathbb{C}}$ is closely related to the $G \times K^{\mathbb{C}}$-orbit structure of $G^{\mathbb{C}}$. Then, slices for the closed $G$-orbits in $G^{\mathbb{C}} / K^{\mathbb{C}}$ can be obtained by applying Matsuki's results [1997, Section 4] on double coset decompositions of groups arising from two involutions.

Let $\mathfrak{k} \oplus \mathfrak{p}$ be the Cartan decomposition of $\mathfrak{g}$ with respect to $K$, and let $\mathfrak{a}$ be a maximal abelian subspace of $\mathfrak{p}$. Following Matsuki, we denote by $\mathscr{A}:=\exp i \mathfrak{a} K^{\mathbb{C}}$ the image of the compact torus $\exp i \mathfrak{a}$ in $G^{\mathbb{C}} / K^{\mathbb{C}}$. The set $\mathscr{A}$ is a slice for all closed $G$-orbits intersecting the compact dual symmetric space $U / K$ in $G^{\mathbb{C}} / K^{\mathbb{C}}$. 
It is called the fundamental Cartan subset. The remaining slices for closed $G$ orbits in $G^{\mathbb{C}} / K^{\mathbb{C}}$ are of the form $\mathscr{C}:=\exp i \mathfrak{c} \cdot z$, where $\mathfrak{c}$ is an abelian semisimple subalgebra of $\mathfrak{g}$ of the same dimension as $\mathfrak{a}$ and $z \in \mathscr{A}$ is a base point sitting on a singular closed $G$-orbit. Such sets $\mathscr{C}$ are called standard Cartan subsets.

By [Geatti 2006], every standard Cartan subset $\mathscr{C}$ admits a base point $z$ with the following properties:

- there exists a subgroup $G^{\prime} \subseteq G$ (possibly $G^{\prime}$ is equal to $G$ ) such that the isotropy subgroup of $z$ in $G^{\prime}$ coincides with the isotropy subgroup $G_{z}$ of $z$ in $G$;

- the quotient $G^{\prime} / G_{z}$ is a pseudo-Riemannian symmetric space of the same rank as $G / K$;

- the slice representation of $G_{z}$ at $z$ is equivalent to the isotropy representation of $G^{\prime} / G_{z}$.

More precisely, let $\mathfrak{g}^{\prime}=\mathfrak{g}_{z} \oplus \mathfrak{q}^{\prime}$ be the decomposition of the Lie algebra of $G^{\prime}$ corresponding to the symmetric space $G^{\prime} / G_{z}$ (when $G^{\prime}=G, \mathfrak{g}=\mathfrak{g}_{z} \oplus \mathfrak{q}$ ). Denote by $T(G \cdot z)_{z}$ the tangent space of the orbit $G \cdot z$ at $z$ and by $N_{z}$ a complementary subspace of $T(G \cdot z)_{z}$ in $T\left(G^{\mathbb{C}} / K^{\mathbb{C}}\right)_{z}$. Then $N_{z} \cong \mathfrak{q}^{\prime}$ and the slice representation at $z$ is equivalent to the Adjoint representation of $G_{z}$ on $\mathfrak{q}^{\prime}$. Moreover, both $\mathfrak{a}$ and $\mathfrak{c}$ are maximal abelian subalgebras in $\mathfrak{q}^{\prime}$.

Consider the twisted bundle $G \times_{G_{z}} \mathfrak{q}^{\prime}$ defined as the orbit space of $G \times \mathfrak{q}^{\prime}$ under the action of $G_{z}$ given by $h \cdot(g, X):=\left(g h^{-1}, \operatorname{Ad}_{h} X\right)$. The group $G$ acts on $G \times{ }_{G_{z}} \mathfrak{q}^{\prime}$ by $\hat{g} \cdot[g, X]:=[\hat{g} g, X]$. By Luna's slice theorem [1975, Proposition 1.2], there exists an open $\operatorname{Ad}_{G_{z}}$-invariant neighborhood $V$ of 0 in $\mathfrak{q}^{\prime}$ such that the map

$$
G \times \times_{G_{z}} V \rightarrow G^{\mathbb{C}} / K^{\mathbb{C}}, \quad[g, X] \mapsto g \exp i X \cdot z
$$

is a $G$-equivariant diffeomorphism onto an open $G$-invariant neighborhood of $z$ in $G^{\mathbb{C}} / K^{\mathbb{C}}$. Nonclosed $G$-orbits in $G \times{ }_{G_{z}} V$ correspond to nonclosed $\operatorname{Ad}_{G_{z}}$-orbits in $V$. The standard Cartan subset $\mathscr{C}$ in $G^{\mathbb{C}} / K^{\mathbb{C}}$ is the image of the set $\{e\} \times \mathfrak{c}$ via the above map.

Let us now assume that $G / K$ has rank one. Then the $G$-orbit space of $G^{\mathbb{C}} / K^{\mathbb{C}}$ can be completely determined. Let $\Delta_{\mathfrak{a}}$ be the restricted root system of $\mathfrak{g}$ with respect to $\mathfrak{a}$, and let

$$
\mathfrak{g}=Z_{\mathfrak{g}}(\mathfrak{a}) \bigoplus_{\alpha \in \Delta_{\mathfrak{a}}} \mathfrak{g}^{\alpha}, \quad \text { with } Z_{\mathfrak{g}}(\mathfrak{a})=Z_{\mathfrak{k}}(\mathfrak{a}) \oplus \mathfrak{a},
$$

be the corresponding root decomposition. Here $Z_{\mathfrak{g}}(\mathfrak{a})$ and $Z_{\mathfrak{k}}(\mathfrak{a})$ denote the centralizers of $\mathfrak{a}$ in $\mathfrak{g}$ and $\mathfrak{k}$, respectively. Let $\Gamma$ be the lattice in $\mathfrak{a}$ given by the kernel of the map $\mathfrak{a} \rightarrow U / K$ defined by $X \mapsto \exp (i X) K$. Since the symmetric space $U / K$ 
is simply connected (see Remark 4.1), the lattice $\Gamma$ is given by

$$
\Gamma=\bigoplus_{\alpha \in \Delta_{\mathfrak{a}}} \mathbb{Z} i \pi h_{\alpha}
$$

where $h_{\alpha} \in \mathfrak{a}$ is uniquely determined by $\alpha\left(h_{\alpha}\right)=2$; see [Helgason 1978, Theorem 8.5, page 322]. Denote by $W_{K}(\mathfrak{a})$ the Weyl group of $\mathfrak{a}$, and let the semidirect product $W_{K}(\mathfrak{a}) \ltimes \Gamma$ act on $\mathfrak{a}$ by $(k, \gamma) \cdot A:=\operatorname{Ad}_{k} A+\gamma$. Denote by $\mathfrak{a}_{0}$ a fundamental domain for this action, and define $\mathscr{A}_{0}:=\exp i \mathfrak{a}_{0} K^{\mathbb{C}}$. Then every closed $G$-orbit through the fundamental Cartan subset $\mathscr{A}$ intersects $\mathscr{A}_{0}$ in a single point; see [Matsuki 1997, Theorem 3].

Let $z \in \mathscr{A}_{0}$ be a base point for a standard Cartan subset $\mathscr{C}$. By [Geatti 2006] and by the local linearization (4-1), the $G$-orbit structure of $G^{\mathbb{C}} / K^{\mathbb{C}}$ in a neighborhood of $z$ is modeled on the orbit structure of the tangent space of a rank-one, pseudoRiemannian symmetric space under the isotropy representation. It can be described as follows.

Remark 4.2. Let $G / H$ a rank-one, pseudo-Riemannian symmetric space. Assume that the group $H$ is connected. Let $\mathfrak{g}=\mathfrak{h} \oplus \mathfrak{q}$ be the corresponding Lie algebra decomposition and $\mathfrak{q} \cap \mathfrak{k} \oplus \mathfrak{q} \cap \mathfrak{p}$ the Cartan decomposition of $\mathfrak{q}$. The isotropy representation of $G / H$ is equivalent to the Adjoint representation of $H$ on $\mathfrak{q}$. Denote by $B$ both the Killing form of $\mathfrak{g}$ and its restriction to $\mathfrak{q} \backslash\{0\}$. The signature of $B$ on $\mathfrak{q}$ is given by $\left(s^{+}, s^{-}\right)$, with

$$
s^{+}:=\operatorname{dim}(\mathfrak{q} \cap \mathfrak{p}) \quad \text { and } \quad s^{-}:=\operatorname{dim}(\mathfrak{q} \cap \mathfrak{k}) .
$$

For $r \in \mathbb{R}$, denote by $B_{r}$ the level hypersurface $\{B=r\}$ in $\mathfrak{q} \backslash\{0\}$. In diagonalized form one has $B_{r}=\left\{x_{1}^{2}+\cdots+x_{s^{+}}^{2}-y_{1}^{2}-\cdots-y_{s^{-}}^{2}=r\right\}$. Since $G / K$ has rank one, every $\operatorname{Ad}_{H}$-orbit in $\mathfrak{q} \backslash\{0\}$ is a hypersurface. Thus, by the connectedness of $H$ and the $\operatorname{Ad}_{H}$-invariance of $B$, such an orbit coincides with a connected component of some $B_{r}$. We distinguish four cases.

(a) Assume $s^{+}=s^{-}=1$. For every $r \neq 0$, the level set $B_{r}$ consists of two connected components. They intersect either $\mathfrak{a}=\mathfrak{q} \cap \mathfrak{p}$ or $\mathfrak{c}=\mathfrak{q} \cap \mathfrak{k}$ in opposite points, depending on whether $r>0$ or $r<0$. The nilcone $B_{0}$ consists of four nonclosed $\mathrm{Ad}_{H}$-orbits.

(b) Assume $s^{+}>1$ and $s^{-}=1$. For $r>0$, the level set $B_{r}$ consists of a single component intersecting $\mathfrak{q} \cap \mathfrak{p}$ in a sphere. Thus, for every nonzero vector $A \in \mathfrak{q} \cap \mathfrak{p}$ and every $t>0$, the points $t A$ and $-t A$ belong to the same $\operatorname{Ad}_{H^{-}}$ orbit. If $r<0$ the level set $B_{r}$ consists of two connected components, which intersect $\mathfrak{c}=\mathfrak{q} \cap \mathfrak{k}$ in opposite points. The nilcone $B_{0}$ consists of two nonclosed $\mathrm{Ad}_{H}$-orbits. 
(c) Assume $s^{+}=1$ and $s^{-}>1$. If $r>0$, the level set $B_{r}$ consists of two connected components, which intersect $\mathfrak{a}=\mathfrak{q} \cap \mathfrak{p}$ in opposite points. If $r<0$, the level set $B_{r}$ intersects $\mathfrak{q} \cap \mathfrak{k}$ in a sphere. Thus for every nonzero vector $C \in \mathfrak{q} \cap \mathfrak{k}$ and every $s>0$, the points $s C$ and $-s C$ belong to the same $\operatorname{Ad}_{H}$-orbit. The nilcone $B_{0}$ consists of two nonclosed $\mathrm{Ad}_{H}$-orbits.

(d) Assume $s^{+}>1$ and $s^{-}>1$. For every $r \neq 0$ the level set $B_{r}$ consists of a single connected component. It intersects either $\mathfrak{q} \cap \mathfrak{p}$ or $\mathfrak{q} \cap \mathfrak{k}$ in a sphere, depending on whether $r>0$ or $r<0$. Thus for every nonzero vector $A \in \mathfrak{q} \cap \mathfrak{p}$ and every $t>0$, the points $t A$ and $-t A$ belong to the same $\operatorname{Ad}_{H}$-orbit. A similar statement holds true for points $s C$ and $-s C$, with $C$ a nonzero vector in $\mathfrak{q} \cap \mathfrak{k}$ and $s>0$. The nilcone $B_{0}$ consists of a unique nonclosed $\mathrm{Ad}_{H}$-orbit.

In order to give further details, we recall the classification of rank-one, Riemannian symmetric spaces of the noncompact type. For each space $M$, we list its real dimension, its standard presentation $G / K$, and the dimensions of the restricted roots spaces of $\mathfrak{g}$; see [Wolf 1984, page 294] and [Helgason 1978, page 532].

\begin{tabular}{llllll}
\hline$M$ & $\operatorname{dim} M$ & $G / K$ & & $\operatorname{dim} \mathfrak{g}^{\alpha}$ & $\operatorname{dim} \mathfrak{g}^{2 \alpha}$ \\
\hline$H^{n}(\mathbb{R})$ & $n$ & $\mathrm{SO}_{0}(n, 1) / \mathrm{SO}(n)$, & $n \geq 2$ & $n-1$ & 0 \\
$H^{n}(\mathbb{C})$ & $2 n$ & $\mathrm{SU}(n, 1) / \mathrm{U}(n)$, & $n \geq 2$ & $2(n-1)$ & 1 \\
$H^{n}(\mathbb{I})$ & $4 n$ & $\mathrm{Sp}(n, 1) / \mathrm{Sp}(n) \times \mathrm{Sp}(1), n \geq 2$ & $4(n-1)$ & 3 \\
$H^{2}(\mathbb{C}$ ay $)$ & 16 & $F_{4}^{*} / \operatorname{Spin}(9)$ & & 8 & 7 \\
\hline
\end{tabular}

Table 4.0

Remark. The two-dimensional symmetric space $\mathrm{SO}_{0}(2,1) / \mathrm{SO}(2)$ can alternately be identified with $\mathrm{SU}(1,1) / \mathrm{U}(1)$ or $\mathrm{SL}(2, \mathbb{R}) / \mathrm{SO}(2)$, and the symmetric space $\mathrm{SO}_{0}(3,1) / \mathrm{SO}(3)$ can be identified with $\mathrm{SL}(2, \mathbb{C}) / \mathrm{SU}(2)$.

4.1. The reduced case. Assume that the restricted root system of $\mathfrak{g}$ is reduced, that is, it consists of two roots $\{ \pm \alpha\}$. This is the case of the spaces $H^{n}(\mathbb{R})$ in Table 4.0. A fundamental domain for the action of $W_{K}(\mathfrak{a}) \ltimes \Gamma$ on $\mathfrak{a}$ is given by $\mathfrak{a}_{0}=\{A \in \mathfrak{a}: 0 \leq \alpha(A) \leq \pi\}$, and there are three singular orbits intersecting $\mathscr{A}_{0}:=\exp i \mathfrak{a}_{0} K^{\mathbb{C}}$. Their base points are given by $z_{j}=g_{j} K^{\mathbb{C}}$ for $j=1,2,3$. Here $g_{j}=\exp i A_{j}$ and the elements $A_{j} \in \mathfrak{a}_{0}$ satisfy the conditions

$$
\alpha\left(A_{1}\right)=0, \quad \alpha\left(A_{2}\right)=\pi / 2, \quad \alpha\left(A_{3}\right)=\pi,
$$

respectively. The $G$-orbits through $z_{1}$ and $z_{3}$ are diffeomorphic to the symmetric space $G / K$ and are embedded in $G^{\mathbb{C}} / K^{\mathbb{C}}$ as totally real submanifolds of maximal dimension. Moreover, the $G$-orbit through $z_{2}$ is a rank-one, pseudo-Riemannian 
symmetric space $G / H$ with involution $\tau_{z_{2}}=\operatorname{Ad}_{g_{2}} \circ \tau \circ \operatorname{Ad}_{g_{2}-1}$. The space $G / H$ is embedded in $G^{\mathbb{C}} / K^{\mathbb{C}}$ as a closed, totally real submanifold of maximal dimension; see [Geatti 2002, Lemma 2.11 and Remark 2.13]. A standard Cartan subset starting at $z_{2}$ is given by $\mathscr{C}=\exp i \mathfrak{c} \cdot z_{2}$, where $\mathfrak{c}=\mathbb{R}(X+\theta(X))$ and $X$ is a nonzero vector in $\mathfrak{g}^{\alpha}$. In the next lemma we determine the $G$-orbit structure of $G^{\mathbb{C}} / K^{\mathbb{C}}$ in a neighborhood of $z_{2}$. Fix a generator $C$ of $\mathfrak{c}$.

Lemma 4.3. Assume that the restricted root system of $\mathfrak{g}$ is reduced. Let $z_{2} \in A_{0}$ be the base point of the Cartan subset $\mathscr{C}$.

(i) If $\operatorname{dim} G / K>2$, then the orbit $G \cdot z_{2}$ is simply connected. In particular, the isotropy subgroup $H$ of $z_{2}$ in $G$ is connected.

(ii) For every $s>0$, the points $\exp (i s C) \cdot z_{2}$ and $\exp (-i s C) \cdot z_{2}$ lie on the same $G$-orbit in $G^{\mathbb{C}} / K^{\mathbb{C}}$ if and only if $\operatorname{dim} \mathfrak{g}^{\alpha}>1$.

(iii) If $\operatorname{dim} \mathfrak{g}^{\alpha}>1$, there are two nonclosed $G$-orbits in $G^{\mathbb{C}} / K^{\mathbb{C}}$ containing $G \cdot z_{2}$ in their closure. If $\operatorname{dim} \mathfrak{g}^{\alpha}=1$, such orbits are four.

Proof. (i) Using the hyperquadric model (see Example 4.4), one can verify that the orbit of $z_{2}$ is diffeomorphic to $\mathrm{SO}_{0}(n, 1) / \mathrm{SO}_{0}(n-1,1)$. In particular, it is topologically equivalent to a sphere of dimension $n-1$ and is simply connected for $n>2$. In that case, the isotropy subgroup $H$ is connected, since $G$ is connected by assumption. When $n=2$, the orbit $G / H$ is not simply connected. The isotropy subgroup of $z_{2}$ is either connected (when $G=\operatorname{SO}_{0}(2,1)$ ) or its quotient by the ineffectivity subgroup is connected (when $G$ is a nontrivial covering of $\operatorname{SO}_{0}(2,1)$ ).

As a result, (ii) and (iii) follow from Remark 4.2, provided that $\operatorname{dim}(\mathfrak{q} \cap \mathfrak{p})=1$ and $\operatorname{dim}(\mathfrak{q} \cap \mathfrak{k})=\operatorname{dim} \mathfrak{g}^{\alpha}$. To show this, define $\mathfrak{g}[\alpha]:=\mathfrak{g}^{\alpha} \oplus \mathfrak{g}^{-\alpha}$. Then $\mathfrak{g}[\alpha]$ is a $\theta$-stable subspace of $\mathfrak{g}$ of dimension equal to $2 \operatorname{dim} \mathfrak{g}^{\alpha}$. Let $\mathfrak{g}[\alpha]=\mathfrak{g}[\alpha]_{\mathfrak{k}} \oplus \mathfrak{g}[\alpha]_{\mathfrak{p}}$ be its Cartan decomposition. The components $\mathfrak{g}[\alpha]_{\mathfrak{k}}$ and $\mathfrak{g}[\alpha]_{\mathfrak{p}}$ are generated by vectors of the form

$$
X+\theta(X) \quad \text { and } \quad X-\theta(X)
$$

respectively, where $X$ ranges through the elements of a basis of $\mathfrak{g}^{\alpha}$. In particular, $\operatorname{dim} \mathfrak{g}[\alpha]_{\mathfrak{k}}=\operatorname{dim} \mathfrak{g}[\alpha]_{\mathfrak{p}}=\operatorname{dim} \mathfrak{g}^{\alpha}$. Consider the decomposition $\mathfrak{g}=Z_{\mathfrak{k}}(\mathfrak{a}) \oplus \mathfrak{a} \oplus \mathfrak{g}[\alpha]$, and note that $\tau_{z_{2}}=\operatorname{Ad}_{g_{2}} \circ \tau \circ \operatorname{Ad}_{g_{2}^{-1}}=\operatorname{Ad}_{g_{2}^{2}} \circ \theta$. Since $\operatorname{Ad}_{\exp i A_{2}}=\exp \left(\operatorname{ad}\left(i A_{2}\right)\right)$, one has $\tau_{z_{2}}=\operatorname{Id}$ on $Z_{\mathfrak{k}}(\mathfrak{a})$ and $\tau_{z_{2}}=-\operatorname{Id}$ on $\mathfrak{a}$. Since $\alpha\left(A_{2}\right)=\pi / 2$, one has $\tau_{z_{2}}=-\theta$ on $\mathfrak{g}[\alpha]$. It follows that $\mathfrak{q}:=\operatorname{Fix}\left(-\tau_{z_{2}}, \mathfrak{g}\right)=\mathfrak{a} \oplus \mathfrak{g}[\alpha]_{\mathfrak{k}}$. In particular, $\operatorname{dim}(\mathfrak{q} \cap \mathfrak{p})=\operatorname{dim} \mathfrak{a}=1$ and $\operatorname{dim}(\mathfrak{q} \cap \mathfrak{k})=\operatorname{dim} \mathfrak{g}[\alpha]_{\mathfrak{k}}=\operatorname{dim} \mathfrak{g}^{\alpha}$, as wished.

From the above discussion and Table 4.0, it follows that in the reduced case the $G$-orbit space of $G^{\mathbb{C}} / K^{\mathbb{C}}$ can be described by the following diagrams. For 
$G / K=\mathrm{SO}_{0}(2,1) / \mathrm{SO}(2)$, the diagram is

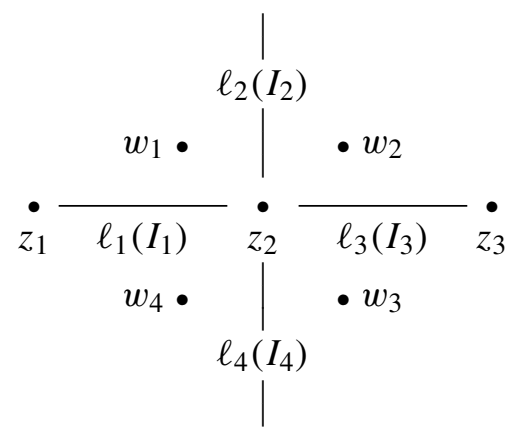

For $G / K=\mathrm{SO}_{0}(n, 1) / \mathrm{SO}(n)$ with $n>2$, the diagram is

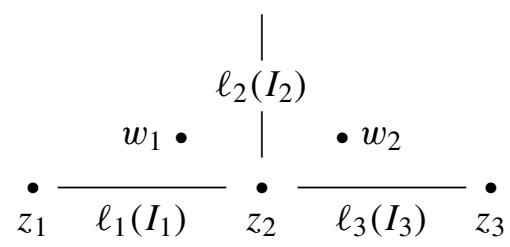

Set $I_{1}=I_{3}=(0,1)$. For $j=1,3$, the maps $\ell_{j}: I_{j} \rightarrow G^{\mathbb{C}} / K^{\mathbb{C}}$, defined by

$$
\ell_{1}(t):=\exp \left(-i t A_{2}\right) \cdot z_{2} \quad \text { and } \quad \ell_{3}(t):=\exp \left(i t A_{2}\right) \cdot z_{2},
$$

parametrize the principal $G$-orbits through $\mathscr{A}_{0}$. One has

$$
\mathscr{A}_{0}=z_{1} \cup \ell_{1}\left(I_{1}\right) \cup z_{2} \cup \ell_{3}\left(I_{3}\right) \cup z_{3} .
$$

Set $I_{2}=I_{4}=(0, \infty)$. For $j=2,4$, the maps $\ell_{j}: I_{j} \rightarrow G^{\mathbb{C}} / K^{\mathbb{C}}$, defined by

$$
\ell_{2}(s):=\exp (i s C) \cdot z_{2} \quad \text { and } \quad \ell_{4}(s):=\exp (-i s C) \cdot z_{2}
$$

parametrize the principal closed $G$-orbits through the standard Cartan subset $\mathscr{C}$ and $\mathscr{C}=\ell_{2}\left(I_{2}\right) \cup z_{2} \cup \ell_{4}\left(I_{4}\right)$. The points $w_{1}, w_{2}, w_{3}$, and $w_{4}$ represent the nonclosed $G$-orbits containing the singular orbit $G \cdot z_{2}$ in their closure.

Example 4.4. The complex hyperquadric. Let $G=\operatorname{SO}_{0}(n, 1)$, with $n \geq 2$, and let $G^{\mathbb{C}}=\operatorname{SO}(n, 1, \mathbb{C})$ be its universal complexification. By definition $G^{\mathbb{C}}$ is the subgroup of $\operatorname{SL}(n+1, \mathbb{C})$ leaving invariant the quadratic form of signature $(n, 1)$. The space $G^{\mathbb{C}} / K^{\mathbb{C}}$ can be identified with the $G^{\mathbb{C}}$-orbit through $(0, \ldots, 1)$ that coincides with the $n$-dimensional complex hyperquadric

$$
M^{\mathbb{C}}=\left\{\left(\xi_{1}, \ldots, \xi_{n+1}\right) \in \mathbb{C}^{n+1}: \xi_{1}^{2}+\cdots+\xi_{n}^{2}-\xi_{n+1}^{2}=-1\right\} .
$$


Fix the elements

$$
A_{2}=\left(\begin{array}{cccc}
0 & \ldots & 0 & 0 \\
\vdots & & \vdots & \vdots \\
0 & \ldots & 0 & \pi / 2 \\
0 & \ldots & \pi / 2 & 0
\end{array}\right) \text { and } C=\left(\begin{array}{ccccc}
0 & \ldots & 0 & 0 & 0 \\
\vdots & & \vdots & \vdots & \vdots \\
0 & \ldots & 0 & -2 & 0 \\
0 & \ldots & 2 & 0 & 0 \\
0 & \ldots & 0 & 0 & 0
\end{array}\right)
$$

in $\mathfrak{g}$ as generators of $\mathfrak{a}$ and $\mathfrak{c}$, respectively. Then points on the singular orbits in $M^{\mathbb{C}}$ satisfying conditions (4-2) are given by

$$
z_{1}=(0, \ldots, 0,1), \quad z_{2}=(0, \ldots, 0, i, 0), \quad z_{3}=(0, \ldots, 0,-1) .
$$

The $G$-orbit of $z_{2}$ is diffeomorphic to the pseudo-Riemannian symmetric space $G / H \cong \mathrm{SO}_{0}(n, 1) / \mathrm{SO}_{0}(n-1,1)$. For $t \in(0,1)$, the slices $\ell_{1}$ and $\ell_{3}$ are given by

$$
\begin{aligned}
& \ell_{1}(t)=(0, \ldots, 0, i \sin (\pi(1-t) / 2), \cos (\pi(1-t) / 2)), \\
& \ell_{3}(t)=(0, \ldots, 0, i \sin (\pi(1+t) / 2), \cos (\pi(1+t) / 2)) .
\end{aligned}
$$

For $s>0$, the slices $\ell_{2}$ and $\ell_{4}$ are given by

$$
\begin{aligned}
& \ell_{2}(s)=(0, \ldots, 0, \quad \sinh 2 s, i \cosh 2 s, 0), \\
& \ell_{4}(s)=(0, \ldots, 0,-\sinh 2 s, i \cosh 2 s, 0) .
\end{aligned}
$$

The slice representation at $z_{2}$ is equivalent to the linear action of $\operatorname{SO}_{0}(n-1,1)$ on $\mathbb{R}^{n}$. When $n=2$, we can choose representatives of the four nonclosed hypersurface $G$-orbits containing $G \cdot z_{2}$ in their closure to be

$$
w_{1}=(-1, i,-1), \quad w_{2}=(1, i,-1), \quad w_{3}=(1, i, 1), \quad w_{4}=(-1, i, 1) .
$$

When $n>2$, the slice representation identifies $\ell_{2}$ and $\ell_{4}$ and representatives of the two nonclosed hypersurface $G$-orbits containing $G \cdot z_{2}$ in their closure are for example

$$
w_{1}=(-1,0, \ldots, 0, i,-1) \text { and } w_{2}=(1,0, \ldots, 0, i,-1) .
$$

4.2. The nonreduced case. Assume that the restricted root system of $\mathfrak{g}$ is nonreduced, that is, it consists of four roots $\{ \pm \alpha, \pm 2 \alpha\}$. This is the case of $H^{n}(\mathbb{C})$, $H^{n}(\mathbb{H})$ and $H^{2}(\mathbb{C} a y)$ in Table 4.0. Then $\mathfrak{a}_{0}=\{A \in \mathfrak{a}: 0 \leq \alpha(A) \leq \pi / 2\}$ is a fundamental domain for the action of $W_{K}(\mathfrak{a}) \ltimes \Gamma$ in $\mathfrak{a}$, and there are three singular orbits intersecting $\mathscr{A}_{0}$. Their base points are given by $z_{j}=g_{j} K^{\mathbb{C}}$ for $j=1,2,3$. Here $g_{j}=\exp i A_{j}$ and the elements $A_{j} \in \mathfrak{a}_{0}$ satisfy the conditions

$$
\alpha\left(A_{1}\right)=0, \quad \alpha\left(A_{2}\right)=\pi / 4, \quad \alpha\left(A_{3}\right)=\pi / 2,
$$


respectively. The $G$-orbit through $z_{1}$ is diffeomorphic to the symmetric space $G / K$, and the one through $z_{3}$ is diffeomorphic to a rank-one, pseudo-Riemannian symmetric space $G / H$. Both orbits are embedded in $G^{\mathbb{C}} / K^{\mathbb{C}}$ as totally real submanifolds of maximal dimension; see [Geatti 2002, Lemma 2.11 and Remark 2.13]. The orbit of $z_{2}$ is a homogeneous space $G / H^{\prime}$, with $H^{\prime}:=G_{z_{2}}$ and $\operatorname{dim} G / H^{\prime}>$ $\operatorname{dim} G / K$; see [Geatti 2002, Lemma 2.14 and Remark 2.15]. Set $G^{\prime}:=Z_{G}\left(g_{2}^{4}\right)$, where $Z_{G}\left(g_{2}^{4}\right)$ denotes the centralizer of $g_{2}^{4}$ in $G$. Then $H^{\prime}$ is contained in $G^{\prime}$ and $G^{\prime} / H^{\prime}$ is a rank-one, pseudo-Riemannian symmetric space with involution $\tau_{z_{2}}=\operatorname{Ad}_{g_{2}} \circ \tau \circ \mathrm{Ad}_{g_{2}^{-1}}$. Moreover, the slice representation at $z_{2}$ is equivalent to the isotropy representation of $G^{\prime} / H^{\prime}$; see [Geatti 2006]. The standard Cartan subset starting at $z_{2}$ is given by $\mathscr{C}^{\prime}=\exp i \mathfrak{c}^{\prime} \cdot z_{2}$, where $\mathfrak{c}^{\prime}=\mathbb{R}(X+\theta(X))$ and $X$ is a nonzero vector in $\mathfrak{g}^{2 \alpha}$. If $Z_{\mathfrak{k}}(\mathfrak{a}) \oplus \mathfrak{a} \oplus \mathfrak{g}^{ \pm \alpha} \oplus \mathfrak{g}^{ \pm 2 \alpha}$ is the restricted root decomposition of $\mathfrak{g}$, then the Lie algebra of $G^{\prime}$ is given by

$$
\mathfrak{g}^{\prime}=Z_{\mathfrak{k}}(\mathfrak{a}) \oplus \mathfrak{a} \oplus \mathfrak{g}^{ \pm 2 \alpha} .
$$

Moreover, if $\mathfrak{h}^{\prime} \oplus \mathfrak{q}^{\prime}$ is the $\tau_{z_{2}}$-decomposition of $\mathfrak{g}^{\prime}$, then $\mathfrak{c}^{\prime}$ is a maximal abelian subalgebra in $\mathfrak{q}^{\prime}$. Fix a generator $C^{\prime}$ of $\mathfrak{c}^{\prime}$.

Lemma 4.5. Assume that the restricted root system of $\mathfrak{g}$ is nonreduced. Let $z_{2} \in \mathscr{A}_{0}$ be the base point of the Cartan subset ' $C^{\prime}$.

(i) The isotropy subgroup $H^{\prime}$ of $z_{2}$ in $G$ is connected.

(ii) For every $t>0$, the points $\exp \left(i t C^{\prime}\right) \cdot z_{2}$ and $\exp \left(-i t C^{\prime}\right) \cdot z_{2}$ sit on the same $G$-orbit if and only if $\operatorname{dim} \mathfrak{g}^{2 \alpha}>1$.

(iii) If $\operatorname{dim} \mathfrak{g}^{2 \alpha}>1$, there are two nonclosed $G$-orbits in $G^{\mathbb{C}} / K^{\mathbb{C}}$ containing $G \cdot z_{2}$ in their closure. If $\operatorname{dim} \mathfrak{g}^{2 \alpha}=1$, such orbits are four.

Proof. (i) The group $H^{\prime}$ is connected if and only if $H^{\prime} \cap K$ is connected. Note that $G^{\prime}=Z_{G}\left(g_{2}^{4}\right)$ is $\theta$-stable, since so is $G$ and $\theta\left(g_{2}^{4}\right)=g_{2}^{-4}$. Therefore $H^{\prime} \cap K$ is the common fixed point subgroup of the two involutions $\tau_{z_{2}}$ and $\theta$ of $G^{\prime}$. As a result, $H^{\prime} \cap K=Z_{K}\left(g_{2}^{2}\right)$. Now regard $z_{2}$ as a point on the compact dual symmetric space $U / K$ endowed with the $K$-action by left translations. Denote by $K_{z_{2}}$ the isotropy subgroup of $z_{2}$ in $K$. On the one hand, $K_{z_{2}}=Z_{K}\left(g_{2}^{2}\right)$. On the other hand, since the isotropy subalgebra $\mathfrak{k}_{z_{2}}$ is given by $\mathfrak{k} \cap \operatorname{Ad}_{z_{2}}(\mathfrak{k})$, one sees that $\mathfrak{k}_{z_{2}}$ has minimal dimension and coincides with $Z_{\mathfrak{k}}(\mathfrak{a})$ if and only if $\alpha\left(A_{2}\right) \neq m \pi$ for $m \in \mathbb{Z}$. By (4-7), it follows that $K_{z_{2}}$ is principal and consequently is equal to $Z_{K}(\mathfrak{a})$. Finally $Z_{K}(\mathfrak{a})$ is connected for all rank-one, Riemannian symmetric spaces of dimension greater than two; see [Knapp 1996] or Lemma 5.1 for a direct proof. In conclusion, $H^{\prime} \cap K=Z_{K}\left(g_{2}^{2}\right)=K_{z_{2}}=Z_{K}(\mathfrak{a})$, which implies (i).

Parts (ii) and (iii) follow by applying Remark 4.2 to the symmetric space $G^{\prime} / H^{\prime}$, provided that $\operatorname{dim} \mathfrak{q}^{\prime} \cap \mathfrak{p}=1$ and $\operatorname{dim} \mathfrak{q}^{\prime} \cap \mathfrak{k}=\operatorname{dim} \mathfrak{g}^{2 \alpha}$. In order to show this, 
define $\mathfrak{g}[2 \alpha]:=\mathfrak{g}^{2 \alpha} \oplus \mathfrak{g}^{-2 \alpha}$. Then $\mathfrak{g}[2 \alpha]$ is $\theta$-stable subspace of $\mathfrak{g}$ of dimension equal to $2 \operatorname{dim} \mathfrak{g}^{2 \alpha}$. Let $\mathfrak{g}[2 \alpha]=\mathfrak{g}[2 \alpha]_{\mathfrak{k}} \oplus \mathfrak{g}[2 \alpha]_{\mathfrak{p}}$ be its Cartan decomposition. The components $\mathfrak{g}[2 \alpha]_{\mathfrak{k}}$ and $\mathfrak{g}[2 \alpha]_{\mathfrak{p}}$ are generated by vectors of the form $X+\theta(X)$ and $X-\theta(X)$, respectively, where $X$ ranges through the elements of a basis of $\mathfrak{g}^{2 \alpha}$. In particular $\operatorname{dim} \mathfrak{g}[2 \alpha]_{\mathfrak{k}}=\operatorname{dim} \mathfrak{g}[2 \alpha]_{\mathfrak{p}}=\operatorname{dim} \mathfrak{g}^{2 \alpha}$. One sees that

$$
\tau_{z_{2}}=\mathrm{Id} \quad \text { on } Z_{\mathfrak{k}}(\mathfrak{a}), \quad \tau_{z_{2}}=-\mathrm{Id} \quad \text { on } \mathfrak{a}, \quad \tau_{z_{2}}=-\theta \quad \text { on } \mathfrak{g}[2 \alpha] .
$$

Consequently $\mathfrak{q}^{\prime}:=\operatorname{Fix}\left(-\tau_{z_{2}}, \mathfrak{g}^{\prime}\right)=\mathfrak{a} \oplus \mathfrak{g}[2 \alpha]_{\mathfrak{k}}$, and $\operatorname{dim}\left(\mathfrak{q}^{\prime} \cap \mathfrak{p}\right)=\operatorname{dim} \mathfrak{a}=1$. Similarly, $\operatorname{dim}\left(\mathfrak{q}^{\prime} \cap \mathfrak{k}\right)=\operatorname{dim} \mathfrak{g}[2 \alpha]_{\mathfrak{k}}=\operatorname{dim} \mathfrak{g}^{2 \alpha}$, as wished.

By [Geatti 2002, Lemma 2.11 and Remark 2.13], the $G$-orbit of $z_{3}$ is a rank-one, pseudo-Riemannian symmetric space $G / H$ with involution $\tau_{z_{3}}=\operatorname{Ad}_{g_{3}} \circ \tau \circ \operatorname{Ad}_{g_{3}-1}$. The space $G / H$ is embedded in $G^{\mathbb{C}} / K^{\mathbb{C}}$ as a closed, totally real submanifold of maximal dimension. The standard Cartan subset that starts at $z_{3}$ is given by $\mathscr{b}=\exp i \mathfrak{c} \cdot z_{3}$, where $\mathfrak{c}=\mathbb{R}(X+\theta(X))$ and $X$ is a nonzero vector in $\mathfrak{g}^{\alpha}$. If $\mathfrak{g}=\mathfrak{h} \oplus \mathfrak{q}$ is the $\tau_{z_{3}}$-decomposition of $\mathfrak{g}$, then $\mathfrak{c}$ is a maximal abelian subalgebra in $\mathfrak{q}$. Fix a generator $C$ of $\mathfrak{c}$.

Lemma 4.6. Assume that the restricted root system of $\mathfrak{g}$ is nonreduced. Let $z_{3} \in \mathscr{A}_{0}$ be the base point of the Cartan subset $\mathscr{C}$.

(i) The orbit $G \cdot z_{3}$ is simply connected. In particular the isotropy subgroup $H$ of $z_{3}$ in $G$ is connected.

(ii) For every $t>0$, the points $\exp (i t C) \cdot z_{3}$ and $\exp (-i t C) \cdot z_{3}$ sit on the same $G$-orbit in $G^{\mathbb{C}} / K^{\mathbb{C}}$.

(iii) There is precisely one nonclosed $G$-orbit in $G^{\mathbb{C}} / K^{\mathbb{C}}$ containing $G \cdot z_{3}$ in its closure.

Proof. (i) Since by assumption $G$ is connected, we prove that $H$ is connected by showing that the orbit $G \cdot z_{3}$ is simply connected. To do this, Remark 4.1 says it suffices to choose $G$ as in the standard presentation in Table 4.0. Let $G=\operatorname{SU}(n, 1)$. By direct computations (see Example 4.7) one finds $G \cdot z_{3} \cong \mathrm{SU}(n, 1) / \mathrm{U}(n-1,1)$. This quotient is topologically equivalent to the complex projective space $\mathbb{C P}^{n-1}$. In particular, it is simply connected.

Consider then $G=\operatorname{Sp}(n, 1)$ or $G=F_{4}^{*}$. In both cases the group $G$ is simply connected. Since $H$ is the fixed point subgroup of an involution of $G$, it is connected [Steinberg 1968]. It follows that the quotient is simply connected.

Parts (ii) and (iii) follow from Remark 4.2, provided that $\operatorname{dim}(\mathfrak{q} \cap \mathfrak{p})=1+\mathfrak{g}^{2 \alpha}$ and $\operatorname{dim}(\mathfrak{q} \cap \mathfrak{k})=\operatorname{dim} \mathfrak{g}^{\alpha}$. In order to show this, define $\mathfrak{g}[\alpha]:=\mathfrak{g}^{\alpha} \oplus \mathfrak{g}^{-\alpha}$ and $\mathfrak{g}[2 \alpha]:=\mathfrak{g}^{2 \alpha} \oplus \mathfrak{g}^{-2 \alpha}$. Then both $\mathfrak{g}[\alpha]$ and $\mathfrak{g}[2 \alpha]$ are $\theta$-stable subspaces of $\mathfrak{g}$ of dimension equal to $\operatorname{dim} \mathfrak{g}^{\alpha}$ and $2 \operatorname{dim} \mathfrak{g}^{2 \alpha}$, respectively. Let $\mathfrak{g}[\alpha]_{\mathfrak{k}}, \mathfrak{g}[\alpha]_{\mathfrak{p}}, \mathfrak{g}[2 \alpha]_{\mathfrak{k}}$, 
and $\mathfrak{g}[2 \alpha]_{\mathfrak{p}}$ be the components of the respective Cartan decompositions. The same arguments as in the proof of Lemmas 4.3 and 4.5 show that

$\operatorname{dim} \mathfrak{g}[\alpha]_{\mathfrak{k}}=\operatorname{dim} \mathfrak{g}[\alpha]_{\mathfrak{p}}=\operatorname{dim} \mathfrak{g}^{\alpha} \quad$ and $\quad \operatorname{dim} \mathfrak{g}[2 \alpha]_{\mathfrak{k}}=\operatorname{dim} \mathfrak{g}[2 \alpha]_{\mathfrak{p}}=\operatorname{dim} \mathfrak{g}^{2 \alpha}$.

Moreover, one sees that

$$
\tau_{z_{3}}=\operatorname{Id} \text { on } Z_{\mathfrak{k}}(\mathfrak{a}), \quad \tau_{z_{3}}=-\operatorname{Id} \text { on } \mathfrak{a}, \quad \tau_{z_{3}}=-\theta \text { on } \mathfrak{g}[\alpha], \quad \tau_{z_{3}}=\theta \text { on } \mathfrak{g}[2 \alpha] .
$$

Since

$$
\mathfrak{g}=Z_{\mathfrak{k}}(\mathfrak{a}) \oplus \mathfrak{a} \oplus \mathfrak{g}[\alpha] \oplus \mathfrak{g}[2 \alpha],
$$

it follows that $\mathfrak{q}:=\operatorname{Fix}\left(-\tau_{z_{3}}, \mathfrak{g}\right)=\mathfrak{a} \oplus \mathfrak{g}[\alpha]_{\mathfrak{k}} \oplus \mathfrak{g}[2 \alpha]_{\mathfrak{p}}$. In particular, $\operatorname{dim}(\mathfrak{q} \cap \mathfrak{p})=$ $1+\operatorname{dim} \mathfrak{g}^{2 \alpha}$ and $\operatorname{dim}(\mathfrak{q} \cap \mathfrak{k})=\operatorname{dim} \mathfrak{g}^{\alpha}$, as claimed.

As a consequence of the above lemmas and Table 4.0, in the nonreduced case the $G$-orbit space of $G^{\mathbb{C}} / K^{\mathbb{C}}$ can be represented by the following diagrams. For $G / K=\mathrm{SU}(n, 1) / \mathrm{U}(n)$ with $n \geq 2$, the diagram is

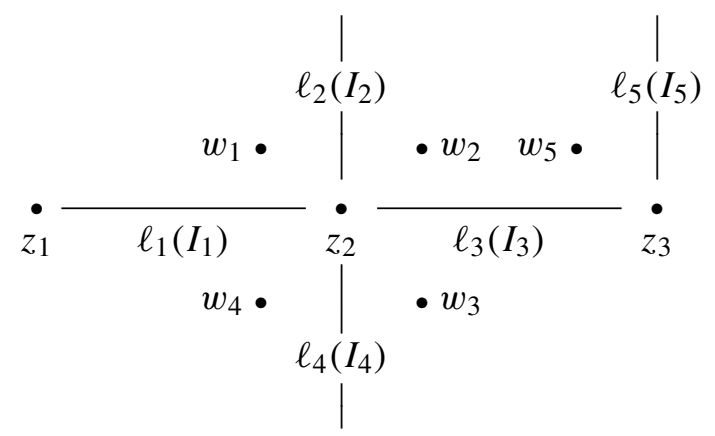

If $G / K=\operatorname{Sp}(n, 1) / \operatorname{Sp}(n) \times \operatorname{Sp}(1)$ for $n \geq 2$, or if $G / K=F_{4}^{*} / \operatorname{Spin}(9)$, then the diagram is

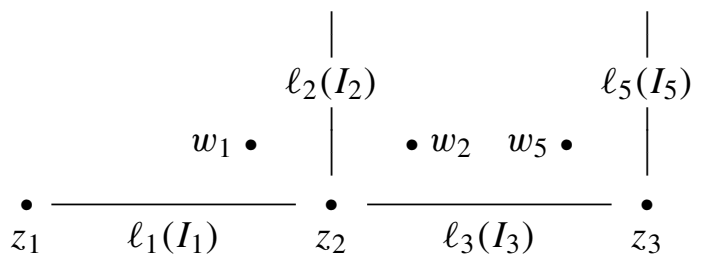

Set $I_{1}=I_{3}=(0,1)$. For $j=1,3$, define $\ell_{j}: I_{j} \rightarrow G^{\mathbb{C}} / K^{\mathbb{C}}$ by

$$
\ell_{1}(t)=\exp \left(-i t A_{2}\right) \cdot z_{2} \quad \text { and } \quad \ell_{3}(t)=\exp \left(i t A_{2}\right) \cdot z_{2} .
$$

The slices $\ell_{1}$ and $\ell_{3}$ parametrize the principal $G$-orbits through $\mathscr{A}_{0}$ and

$$
\mathscr{A}_{0}=z_{1} \cup \ell_{1}\left(I_{1}\right) \cup z_{2} \cup \ell_{3}\left(I_{3}\right) \cup z_{3} .
$$


Set $I_{2}=I_{4}=(0, \infty)$. For $j=2,4$, define $\ell_{j}: I_{j} \rightarrow G^{\mathbb{C}} / K^{\mathbb{C}}$ by

$$
\ell_{2}(s)=\exp \left(s C^{\prime}\right) \cdot z_{2} \quad \text { and } \quad \ell_{4}(s)=\exp \left(-s C^{\prime}\right) \cdot z_{2} .
$$

The slices $\ell_{2}$ and $\ell_{4}$ parametrize the principal $G$-orbits through the Cartan subset $\mathscr{C}^{\prime}$ with base point $z_{2}$ and $\mathscr{C}^{\prime}=\ell_{2}\left(I_{2}\right) \cup z_{2} \cup \ell_{4}\left(I_{4}\right)$. Finally, set $I_{5}=(0, \infty)$, and define $\ell_{5}: I_{5} \rightarrow G^{\mathbb{C}} / K^{\mathbb{C}}$ by

$$
\ell_{5}(s)=\exp (s C) \cdot z_{3} .
$$

The slice $\ell_{5}$ parametrizes the principal $G$-orbits through the standard Cartan subset $\mathscr{C}$ with base point $z_{3}$. The points $w_{1}, \ldots, w_{4}$ represent the nonclosed orbits containing $G \cdot z_{2}$ in their closure. The point $w_{5}$ represents the nonclosed orbit containing $G \cdot z_{3}$ in its closure.

Example 4.7. A model in the nonreduced case. Let $G=\operatorname{SU}(n, 1)$, with $n \geq 2$, be the subgroup of $\operatorname{SL}(n+1, \mathbb{C})$ leaving invariant the hermitian form $\langle z, w\rangle_{n, 1}=$ $z_{1} \bar{w}_{1}+\ldots+z_{n} \bar{w}_{n}-z_{n+1} \bar{w}_{n+1}$ in $\mathbb{C}^{n+1}$. Denote by $\sigma$ the conjugation of $G^{\mathbb{C}}=$ $\operatorname{SL}(n+1, \mathbb{C})$ relative to $G$, namely $\sigma(g)=I_{n, 1}{ }^{t} \bar{g}^{-1} I_{n, 1}$. Denote by $\overline{\mathbb{P}}^{n}$ the complex projective space endowed with the opposite complex structure, that is, the one for which the map $\mathbb{P}^{n} \rightarrow \overline{\mathbb{P}}^{n},[z] \mapsto[\bar{z}]$ is holomorphic. The group $G^{\mathbb{C}}$ acts holomorphically on $\mathbb{P}^{n} \times \overline{\mathbb{P}}^{n}$ by $g \cdot([z],[w]):=([g \cdot z],[\sigma(g) \cdot w])$.

Under this action, $\mathbb{P}^{n} \times \overline{\mathbb{P}}^{n}$ consists of two orbits: a closed one given by

$$
\left\{([z],[w]) \in \mathbb{P}^{n} \times \overline{\mathbb{P}}^{n}:\langle z, w\rangle_{n, 1}=0\right\}
$$

and an open one given by its complement. The quotient $G^{\mathbb{C}} / K^{\mathbb{C}}$ can be identified with the open orbit

$$
M^{\mathbb{C}}:=G^{\mathbb{C}} \cdot([0: \cdots: 0: 1],[0: \cdots: 0: 1])=\mathbb{P}^{n} \times \overline{\mathbb{P}}^{n} \backslash\left\{\langle z, w\rangle_{n, 1}=0\right\} .
$$

Fix the elements

$$
A_{2}=\left(\begin{array}{cccc}
0 & \ldots & 0 & 0 \\
\vdots & & \vdots & \vdots \\
0 & \ldots & 0 & \pi / 4 \\
0 & \ldots & \pi / 4 & 0
\end{array}\right), \quad C^{\prime}=\left(\begin{array}{cccc}
0 & \ldots & 0 & 0 \\
\vdots & & \vdots & \vdots \\
0 & \ldots & i & 0 \\
0 & \ldots & 0 & -i
\end{array}\right), \quad C=\left(\begin{array}{ccccc}
0 & \ldots & 0 & 0 & 0 \\
\vdots & & \vdots & \vdots & \vdots \\
0 & \ldots & 0 & -1 & 0 \\
0 & \ldots & 1 & 0 & 0 \\
0 & \ldots & 0 & 0 & 0
\end{array}\right)
$$

in $\mathfrak{g}$ as generators of $\mathfrak{a}, \mathfrak{c}^{\prime}$ and $\mathfrak{c}$, respectively. Then points on the singular orbits in $M^{\mathbb{C}}$ satisfying conditions (4-7) are given by

$$
\begin{aligned}
z_{1} & =([0: \ldots: 0: 1],[0: \ldots: 0: 1]), \\
z_{2} & =([0: \ldots: 0: i: 1],[0: \ldots: 0:-i: 1]), \\
z_{3} & =([0: \ldots: 0: 1: 0],[0: \ldots: 0: 1: 0]) .
\end{aligned}
$$


The $G$-orbit of $z_{2}$ is diffeomorphic to the homogeneous space $G / H^{\prime}$, where $H^{\prime} \cong$ $\mathrm{U}(n-1) \times \mathrm{SO}(1,1)$. The group $G^{\prime}$ is isomorphic to $\mathrm{U}(n-1) \times \mathrm{SU}(1,1)$, and the quotient $G^{\prime} / H^{\prime}$ is diffeomorphic to the two-dimensional rank-one, pseudoRiemannian symmetric space $\mathrm{SU}(1,1) / \mathrm{SO}(1,1)$. The $G$-orbit of $z_{3}$ is diffeomorphic to the pseudo-Riemannian symmetric space $\operatorname{SU}(n, 1) / \mathrm{SU}(n-1,1)$. The slices $\ell_{1}$ and $\ell_{3}$ are given by

$$
\begin{aligned}
& \ell_{1}(t)=\left(\left[0: \ldots: i \sin \frac{\pi}{4}(1-t): \cos \frac{\pi}{4}(1-t)\right],\left[0: \ldots:-i \sin \frac{\pi}{4}(1-t): \cos \frac{\pi}{4}(1-t)\right]\right), \\
& \ell_{3}(t)=\left(\left[0: \ldots: i \sin \frac{\pi}{4}(1+t): \cos \frac{\pi}{4}(1+t)\right],\left[0: \ldots:-i \sin \frac{\pi}{4}(1+t): \cos \frac{\pi}{4}(1+t)\right]\right),
\end{aligned}
$$

where $t \in(0,1)$. The slices $\ell_{2}, \ell_{4}$ and $\ell_{5}$ are given by

$$
\begin{aligned}
& \ell_{2}(s)=\left(\left[0: \ldots: i e^{-s}: e^{s}\right],\left[0: \ldots:-i e^{s}: e^{-s}\right]\right), \\
& \ell_{4}(s)=\left(\left[0: \ldots: i e^{s}: e^{-s}\right],\left[0: \ldots:-i e^{-s}: e^{s}\right]\right), \\
& \ell_{5}(s)=([0: \ldots: \sinh s: i \cosh s: 0],[0: \ldots: \sinh s:-i \cosh s: 0]),
\end{aligned}
$$

with $s>0$. The slice representation at $z_{2}$ is equivalent to the standard action of $\operatorname{SO}(1,1)$ on $\mathbb{R}^{2}$. So there are four nonclosed $G$-orbits containing $G \cdot z_{2}$ in their closure. We can choose representatives of such orbits to be

$$
\begin{array}{ll}
w_{1}=([0: \ldots: 0: 1],[0: \ldots:-i: 1]), & w_{2}=([0: \ldots: i: 1],[0: \ldots: 1: 0]), \\
w_{3}=([0: \ldots: 1: 0],[0: \ldots:-i: 1]), & w_{4}=([0: \ldots: i: 1],[0: \ldots: 0: 1]) .
\end{array}
$$

A representative for the unique nonclosed orbit containing $G \cdot z_{3}$ in its closure is given by $w_{5}=([0: \ldots: 1:-i: 1],[0: \ldots: 1: i: 1])$.

Remark 4.8. When $G=\mathrm{SU}(1,1)$, the restricted root system of $\mathfrak{g}$ is reduced. The quotient $G^{\mathbb{C}} / K^{\mathbb{C}}$ can be identified with $\mathbb{P}^{1} \times \overline{\mathbb{P}}^{1} \backslash\left\{\langle z, w\rangle_{1,1}=0\right\}$, and the $G$ orbit space can be described as above, except for the fact that the slice $\ell_{5}$ and the point $w_{5}$ must be omitted. Moreover the $G$-orbit through $z_{3}$ is diffeomorphic to the symmetric space $G / K$. Note that $\mathrm{SU}(1,1)^{\mathbb{C}} / \mathrm{U}(1)^{\mathbb{C}}$ is biholomorphic to $\mathrm{SO}_{0}(2,1)^{\mathbb{C}} / \mathrm{SO}(2)^{\mathbb{C}}$. Thus it can also be identified with the two-dimensional hyperquadric described in Example 4.4.

\section{Univalence on $G$-orbits in $G^{\mathbb{C}} / K^{\mathbb{C}}$}

Let $G$ be a connected, noncompact, real simple Lie group, let $K \subset G$ be a maximal compact subgroup, and let $G^{\mathbb{C}}$ be the universal complexification of $G$. Assume that $G$ is embedded in $G^{\mathbb{C}}$. Consider a $G$-equivariant Riemann domain

$$
q: \Sigma \rightarrow G^{\mathbb{C}} / K^{\mathbb{C}} .
$$

The main goal of this section is to prove that $q$ is injective on $G$-orbits if $G / K$ is a rank-one, Riemannian symmetric space of dimension greater than three. We 
first prove the result for principal $G$-orbits, and later we extend it to all $G$-orbits by a general argument. In most cases, the injectivity of $q$ on principal $G$-orbits follows from their simple connectedness. The cases $\operatorname{dim} G / K=2,3$ are discussed separately.

Recall that by Remark 4.1(ii), different triples $\left(G, K, G^{\mathbb{C}}\right)$ associated with the same Riemannian symmetric space $G / K$ yield $G$-equivariantly diffeomorphic orbits in $G^{\mathbb{C}} / K^{\mathbb{C}}$. Let $\mathscr{A}_{0}, \mathscr{C}^{\prime}$ and $\mathscr{C}$ be the standard Cartan subsets in $G^{\mathbb{C}} / K^{\mathbb{C}}$. Let $H$ be the isotropy subgroup of the base point of $\mathscr{C}$, and let $H^{\prime}$ be the isotropy subgroup of the base point of $\mathscr{C}^{\prime}$; see Lemmas 4.3, 4.5 and 4.6. By [Geatti 2002, Propositions 3.4 and 3.15], the principal orbits intersecting $\mathscr{A}_{0}, \mathscr{C}$ and $\mathscr{C}^{\prime}$ have isotropy type $Z_{K}(\mathfrak{a}), Z_{H}(\mathfrak{c})$ and $Z_{H^{\prime}}\left(\mathfrak{c}^{\prime}\right)$, respectively.

Lemma 5.1. Principal $G$-orbits of isotropy type $Z_{K}(\mathfrak{a})$ are simply connected if and only if $\operatorname{dim} G / K>2$.

Proof. An orbit $G / Z_{K}(\mathfrak{a})$ is topologically equivalent to $K / Z_{K}(\mathfrak{a})$. Consider the isotropy representation of $K$ on $\mathfrak{p}$. The nonzero $K$-orbits in $\mathfrak{p}$ are diffeomorphic to $K / Z_{K}(\mathfrak{a})$. Since $G / K$ has rank one, they are also diffeomorphic to spheres of $\operatorname{dimension} \operatorname{dim}(G / K)-1$. Hence the statement follows.

Remark 5.2. When $G=\operatorname{SO}_{0}(2,1)$, the isotropy subgroup $Z_{K}(\mathfrak{a})$ is trivial. Therefore principal orbits of type $G / Z_{K}(\mathfrak{a})$ are diffeomorphic to $\mathrm{SO}_{0}(2,1)$ and topologically equivalent to $\mathrm{SO}(2)$. In particular, they are not simply connected.

Lemma 5.3. Principal $G$-orbits of isotropy type $Z_{H}(\mathfrak{c})$ are simply connected, except when $G$ is one of the groups $\mathrm{SO}_{0}(2,1), \mathrm{SO}_{0}(3,1)$ or $\mathrm{SU}(2,1)$.

Proof. An orbit $G / Z_{H}(\mathfrak{c})$ is topologically equivalent to $K / Z_{K \cap H}(\mathfrak{c})$. We prove the lemma by discussing each case separately. Let $G=\operatorname{SO}_{0}(n, 1)$. Using the hyperquadric model given in Example 4.4, one checks that

$$
H \cong \mathrm{SO}_{0}(n-1,1), \quad Z_{H}(\mathfrak{c}) \cong \mathrm{SO}_{0}(n-2,1), \quad K / Z_{H \cap K}(\mathfrak{c}) \cong \mathrm{SO}(n) / \mathrm{SO}(n-2) .
$$

In particular, $K / Z_{K \cap H}(\mathfrak{c})$ is diffeomorphic to a Stiefel manifold, which is simply connected for $n>3$.

Consider next the case $G=\mathrm{SU}(n, 1)$, with $n \geq 3$. Direct computations on the model in Example 4.7 show that

$$
\begin{aligned}
H & \cong \mathrm{U}(n-1,1), \\
Z_{K \cap H}(\mathfrak{c}) & \cong \mathrm{U}(n-2) \times \mathrm{U}(1), \\
K / Z_{K \cap H}(\mathfrak{c}) & \cong \mathrm{U}(n) /(\mathrm{U}(n-2) \times \mathrm{U}(1)) .
\end{aligned}
$$

Since, for $n \geq 3$, the embedding $\mathrm{U}(n-2) \rightarrow \mathrm{U}(n)$ induces an epimorphism of fundamental groups, so does the embedding $\mathrm{U}(n-2) \times \mathrm{U}(1) \rightarrow \mathrm{U}(n)$. As a consequence, $K / Z_{K \cap H}(\mathfrak{c})$ is simply connected. 
Finally, consider $G=\operatorname{Sp}(n, 1)$ or $G=F_{4}^{*}$. Note that in both cases $K$ is simply connected. Therefore $K / Z_{K \cap H}(\mathfrak{c})$ is simply connected provided that $Z_{K \cap H}(\mathfrak{c})$ is connected. In order to show that this, consider the compact, rank-one, symmetric space $K / K \cap H$ and the corresponding isotropy representation of $K \cap H$ on $\mathfrak{k} \cap \mathfrak{q}$. The nonzero $K \cap H$-orbits in $\mathfrak{k} \cap \mathfrak{q}$ are of type $K \cap H / Z_{K \cap H}(\mathfrak{c})$ and are diffeomorphic to spheres of dimension $\operatorname{dim}(\mathfrak{k} \cap \mathfrak{q})-1$. Since $\operatorname{dim}(\mathfrak{k} \cap \mathfrak{q})=\operatorname{dim} \mathfrak{g}^{\alpha}>2$ (see Table 4.0), they are simply connected. By Lemma 4.3 or Lemma 4.6, the group $H$ and likewise its maximal compact subgroup $K \cap H$ are connected. Then the exact homotopy sequence of the quotient $K \cap H / Z_{K \cap H}(\mathfrak{c})$, implies that the group $Z_{K \cap H}(\mathfrak{c})$ is connected, as wished.

Remark 5.4. When $G=\operatorname{SO}_{0}(2,1)$, direct computations using the model described in Example 4.4 show that the isotropy subgroup $Z_{H}(\mathfrak{c})$ is trivial. Therefore principal orbits of type $G / Z_{H}(\mathfrak{c})$ are diffeomorphic to $\mathrm{SO}_{0}(2,1)$ and topologically equivalent to $\mathrm{SO}(2)$. In particular, they are not simply connected.

Similarly, when $G=\mathrm{SO}_{0}(3,1)$ the isotropy subgroup $Z_{H}(\mathfrak{c})$ is isomorphic to $\mathrm{SO}_{0}(1,1)$, which is connected. Therefore principal orbits of type

$$
G / Z_{H}(\mathfrak{c}) \cong \mathrm{SO}_{0}(3,1) / \mathrm{SO}_{0}(1,1)
$$

are topologically equivalent to $\mathrm{SO}(3)$ and are not simply connected.

When $G=\mathrm{SU}(2,1)$, direct computations using the model described in Example 4.7 show that the isotropy subgroup $Z_{K \cap H}(\mathfrak{c})$ is isomorphic to $S(\mathrm{U}(1) \times \mathrm{U}(1))$, which is connected. Principal orbits of type $G / Z_{H}(\mathfrak{c})$ are topologically equivalent to $K / Z_{K \cap H}(\mathfrak{c}) \cong \mathrm{U}(2) / \mathrm{U}(1) \cong \mathrm{SO}(3)$. Hence they are not simply connected.

Note that in all the above cases, despite the fact that the orbits are not simply connected, the corresponding isotropy subgroups are connected.

\section{Lemma 5.5. All principal $G$-orbits of type $Z_{H^{\prime}}\left(\mathfrak{c}^{\prime}\right)$ are simply connected.}

Proof. An orbit of type $G / Z_{H^{\prime}}\left(\mathfrak{c}^{\prime}\right)$ is topologically equivalent to $K / Z_{H^{\prime} \cap K}\left(\mathfrak{c}^{\prime}\right)$. We prove the latter quotient is simply connected by discussing each case separately.

Consider first $G=\mathrm{SU}(n, 1)$. Direct computations using the model constructed in Example 4.7 show that $Z_{H^{\prime} \cap K}\left(\mathfrak{c}^{\prime}\right) \cong \mathrm{U}(n-1)$. Hence the quotient $K / Z_{H^{\prime} \cap K}\left(\mathfrak{c}^{\prime}\right) \cong$ $\mathrm{U}(n) / \mathrm{U}(n-1)$ is diffeomorphic to the sphere $S^{2 n-1}$. In particular, it is simply connected for all $n \geq 2$.

Next let $G=\operatorname{Sp}(n, 1)$ or $G=F_{4}^{*}$. Both $G$ and $K$ are simply connected. So the quotient $K / Z_{H^{\prime} \cap K}\left(\mathfrak{c}^{\prime}\right)$ is simply connected provided that $Z_{H^{\prime} \cap K}\left(\mathfrak{c}^{\prime}\right)$ is connected. In order to show this, denote by $K^{\prime}$ the maximal compact subgroup of $G^{\prime}$; see Section 4.2. Since $H^{\prime}$ is contained in $G^{\prime}$, the groups $H^{\prime} \cap K$ and $H^{\prime} \cap K^{\prime}$ coincide and are both connected by Lemma 4.5. Consider the compact, rank-one, symmetric space $K^{\prime} /\left(K^{\prime} \cap H^{\prime}\right) \subset G^{\prime} / H^{\prime}$. The nonzero orbits of the isotropy representation of $K^{\prime} \cap H^{\prime}$ on $\mathfrak{k}^{\prime} \cap \mathfrak{q}^{\prime}$ are of type $K^{\prime} \cap H^{\prime} / Z_{K^{\prime} \cap H^{\prime}}\left(\mathfrak{c}^{\prime}\right)$ and are diffeomorphic to 
spheres of dimension equal to $\operatorname{dim} \mathfrak{g}^{2 \alpha}-1$. Since $\operatorname{dim} \mathfrak{g}^{2 \alpha}>2$ (see Table 4.0), they are simply connected. Since $H^{\prime} \cap K^{\prime}$ is connected, it follows from the exact homotopy sequence of the quotient $K^{\prime} \cap H^{\prime} / Z_{K^{\prime} \cap H^{\prime}}\left(\mathfrak{c}^{\prime}\right)$ that the groups $Z_{K^{\prime} \cap H^{\prime}}\left(\mathfrak{c}^{\prime}\right)$ and $Z_{K \cap H^{\prime}}\left(\mathfrak{c}^{\prime}\right)$ are also connected. It follows that the quotients $K / Z_{H^{\prime} \cap K}\left(\mathfrak{c}^{\prime}\right)$ and $G / Z_{H^{\prime} \cap K}\left(\mathfrak{c}^{\prime}\right)$ are simply connected, as desired.

Lemma 5.6. Let $q: \Sigma \rightarrow Z$ be a G-equivariant Riemann domain. Assume that every $z$ in $Z$ admits an arbitrary small neighborhood $V$ and a sequence $\left\{z_{n}\right\}$ converging to $z$ with the property that both the isotropy subgroups $G_{z_{n}}$ and the intersections $G \cdot z_{n} \cap V$ are connected. Then $q$ is injective on every $G$-orbit of $\Sigma$.

Proof. Assume by contradiction that the map $q$ is not injective on the $G$-orbit through some $\zeta$ in $\Sigma$. Then there exists $h \in G$ with $h \cdot \zeta \neq \zeta$ such that $q(h \cdot \zeta)=q(\zeta)$. Since $q$ is locally injective, one can choose an open neighborhood $V$ of $z:=q(\zeta)$ in $Z$ as in the assumption, and open neighborhoods $W_{\zeta}$ and $W_{h \cdot \zeta}$ of $\zeta$ and $h \cdot \zeta$ in $\Sigma$, such that $W_{\zeta} \cap W_{h \cdot \zeta}=\varnothing$ and the restrictions $q \mid W_{\zeta}: W_{\zeta} \rightarrow V$ and $q \mid W_{h \cdot \zeta}: W_{h \cdot \zeta} \rightarrow V$ are bijective. Then there exists a sequence $\left\{z_{n}\right\}$ in $Z$, converging to $z$, with the property that both the isotropy subgroups $G_{z_{n}}$ and the intersections $G \cdot z_{n} \cap V$ are connected.

Consider the sequence $\left\{\zeta_{n}:=\left(q \mid W_{\zeta}\right)^{-1}\left(z_{n}\right)\right\}$ in $W_{\zeta}$. Since $\left\{\zeta_{n}\right\}$ converges to $\zeta$ for $n$ large enough, the points $h \cdot \zeta_{n}$ lie in $W_{h \cdot \zeta}$. Therefore their images $q\left(h \cdot \zeta_{n}\right)=$ $h \cdot q\left(\zeta_{n}\right)=h \cdot z_{n}$ lie in $V$. Since both $G_{z_{n}}$ and $G \cdot z_{n} \cap V$ are connected, the set $\Omega_{n}:=\left\{g \in G: g \cdot z_{n} \in V\right\}$ is connected. Note that both $e$ and $h$ belong to $\Omega_{n}$. Hence there exists a continuous path $\gamma:[0,1] \rightarrow \Omega_{n}$ with $\gamma(0)=e$ and $\gamma(1)=h$. By the $G$-equivariance of $q$, both paths $t \mapsto\left(q \mid W_{\zeta}\right)^{-1}\left(\gamma(t) \cdot z_{n}\right)$ and $t \mapsto \gamma(t) \cdot \zeta_{n}$ in $\Sigma$ are liftings of $t \mapsto \gamma(t) \cdot z_{n}$, with initial point $\zeta_{n}$. On the other hand, $\left(q \mid W_{\zeta}\right)^{-1}\left(\gamma(1) \cdot z_{n}\right) \in W_{\zeta}$ while $\gamma(1) \cdot \zeta_{n} \in W_{h \cdot \zeta}$, giving a contradiction.

As a consequence of these lemmas, we obtain the main result of this section.

Proposition 5.7. Let $G$ be a connected, noncompact, real simple Lie group such that the Riemannian symmetric space $G / K$ has rank one. Assume that $G$ is embedded in its universal complexification $G^{\mathbb{C}}$ and is different from the groups $\operatorname{SL}(2, \mathbb{R})$ and $\operatorname{Spin}(3,1)$. Let $q: \Sigma \rightarrow G^{\mathbb{C}} / K^{\mathbb{C}}$ be a $G$-equivariant Riemann domain. Then $q$ is injective on every $G$-orbit.

Proof. We begin by proving the following claim.

Claim. The isotropy subgroups of all principal $G$-orbits are connected.

Proof of the claim. Since $G$ is connected, the isotropy subgroups of simply connected orbits are necessarily connected. Hence by Lemmas 5.1-5.5 we only need to discuss the isotropy types $Z_{K}(\mathfrak{a})$ when $G$ has Lie algebra $\mathfrak{s o}_{0}(2,1)$ and the isotropy types $Z_{H}(\mathfrak{c})$ when $G$ has Lie algebra $\mathfrak{s o}_{0}(2,1), \mathfrak{s o}_{0}(3,1)$ and $\mathfrak{s u}(2,1)$. 
Let $\mathfrak{g}=\mathfrak{s o}(2,1)$. When $G=\mathrm{SO}_{0}(2,1)$ the isotropy subgroups of all principal $G$-orbits are connected, by Remarks 5.2 and 5.4. Observe that $\operatorname{SO}_{0}(2,1)$ is centerless and that $\mathrm{SL}(2, \mathbb{R})$ is a double covering of $\mathrm{SO}_{0}(2,1)$. Since the universal complexification of $\operatorname{SL}(2, \mathbb{R})$ is $\operatorname{SL}(2, \mathbb{C})$, which is simply connected, no covering of $\mathrm{SO}_{0}(2,1)$ other than $\mathrm{SL}(2, \mathbb{R})$ admits an embedding into its universal complexification. Hence the claim follows for every group $G \neq \operatorname{SL}(2, \mathbb{R})$ that has Lie algebra $\mathfrak{s o}(2,1)$ and embeds in its universal complexification.

Let $\mathfrak{g}=\mathfrak{s o}(3,1)$. When $G=\mathrm{SO}_{0}(3,1)$ the isotropy subgroup $Z_{H}(\mathfrak{c})$ is connected, by Remark 5.4. Note that $\operatorname{SO}_{0}(3,1)$ is centerless and $\operatorname{Spin}(3,1)$ is the only nontrivial covering of $\mathrm{SO}_{0}(3,1)$ that embeds in its universal complexification. Hence the claim follows for every group $G \neq \operatorname{Spin}(3,1)$ that has Lie algebra $\mathfrak{s o}(3,1)$ and embeds in its universal complexification.

Finally, let $\mathfrak{g}=\mathfrak{s u}(2,1)$. When $G=\operatorname{SU}(2,1)$, the isotropy subgroup $Z_{H}(\mathfrak{c})$ is connected, by Remark 5.4. Thus the same holds true for every connected real Lie group covered by $\mathrm{SU}(2,1)$. Since no covering group of $\mathrm{SU}(2,1)$ admits an embedding in its universal complexification, the claim holds true for every $G$ that has Lie algebra $\mathfrak{s u}(2,1)$ and embeds in its universal complexification. This concludes the proof of the claim.

In order to complete the proof of the proposition, recall that the union of principal $G$-orbits forms an open dense subset of $G^{\mathbb{C}} / K^{\mathbb{C}}$. Hence, by the above claim every point in $G^{\mathbb{C}} / K^{\mathbb{C}}$ can be approximated by points with connected isotropy subgroups. Due to this fact and the description of the slice representation at closed $G$-orbits (see Remark 4.2 and the diagrams in Section 4), all assumptions of Lemma 5.6 are met and the statement follows.

Remark 5.8. When $G=\operatorname{SL}(2, \mathbb{R})$, the isotropy subgroups of all principal $G$-orbits in $G^{\mathbb{C}} / K^{\mathbb{C}}$ consist of the central elements $\left\{ \pm I_{2}\right\}$. As we shall see in Example 7.7, in this case there exist Stein, $G$-equivariant Riemann domains that are not injective on $G$-orbits. Similarly, one can construct $G$-equivariant Riemann domains that are not injective on $G$-orbits in the case $G=\operatorname{Spin}(3,1)$. However, by Theorem 7.6 such Riemann domains cannot be Stein.

\section{6. $G$-invariant Stein domains in $G^{\mathbb{C}} / K^{\mathbb{C}}$}

Let $G / K$ be a noncompact, rank-one, Riemannian symmetric space. In this section we exhibit a complete classification of Stein $G$-invariant domains in $G^{\mathbb{C}} / K^{\mathbb{C}}$. The main ingredient is the computation of the Levi form of hypersurface $G$-orbits in $G^{\mathbb{C}} / K^{\mathbb{C}}$, which is carried out in [Geatti 2002] and in the appendix, Section 9. Most of the Stein domains in our list are known. However, for $G=\mathrm{SU}(n, 1)$ we present some examples which appear to be new. By working out an explicit model of $G^{\mathbb{C}} / K^{\mathbb{C}}$, we show that they are all biholomorphic to $\mathbb{B}^{n} \times \mathbb{C}^{n}$. 
The classification result is stated for the standard presentations of $G / K$ given in Table 4.0. This is no loss of generality, since by Remark 4.1 the $G$-orbit structure of $G^{\mathbb{C}} / K^{\mathbb{C}}$ as well as the CR-structure and topology of $G$-orbits do not depend on the presentation of the symmetric space $G / K$.

Retain the notation used in diagrams (4-3), (4-4), (4-9), and (4-10). Consider the $G$-invariant domains in $G^{\mathbb{C}} / K^{\mathbb{C}}$ defined, for $0 \leq a<1$ and $0 \leq b<\infty$, by

$$
\begin{aligned}
D_{1}(a) & =G \cdot\left(z_{1} \cup \ell_{1}((a, 1))\right), & D_{2}(a) & =G \cdot\left(z_{3} \cup \ell_{3}((a, 1))\right), \\
S_{1}(b) & =G \cdot \ell_{2}((b, \infty)), & S_{2}(b) & =G \cdot \ell_{4}((b, \infty)),
\end{aligned}
$$

Theorem 6.1. Let $G / K$ be a noncompact, rank-one, Riemannian symmetric space.

\begin{tabular}{|c|c|c|c|c|c|}
\hline Domain & $G=$ & $\mathrm{SO}_{0}(2,1)$ & $\begin{array}{c}\mathrm{SO}_{0}(n, 1) \\
n \geq 3\end{array}$ & $\begin{array}{c}\mathrm{SU}(n, 1) \\
n \geq 2\end{array}$ & $\begin{array}{l}\operatorname{Sp}(n, 1), n \geq 2 \\
F_{4}^{*}\end{array}$ \\
\hline$D_{1}(a)$ & $0 \leq a<1$ & Stein & Stein & Stein & Stein \\
\hline$D_{2}(a)$ & $0 \leq a<1$ & Stein & Stein & no & no \\
\hline$S_{1}(b)$ & $0 \leq b<\infty$ & Stein & no & no & no \\
\hline$S_{2}(b)$ & $0 \leq b<\infty$ & Stein & no & no & no \\
\hline \multicolumn{2}{|c|}{$D_{1}(0) \cup G \cdot w_{1} \cup S_{1}(0)$} & Stein & no & Stein & no \\
\hline \multicolumn{2}{|c|}{$D_{1}(0) \cup G \cdot w_{4} \cup S_{2}(0)$} & Stein & no & Stein & no \\
\hline \multicolumn{2}{|c|}{$D_{2}(0) \cup G \cdot w_{2} \cup S_{1}(0)$} & Stein & no & no & no \\
\hline \multicolumn{2}{|c|}{$D_{2}(0) \cup G \cdot w_{3} \cup S_{2}(0)$} & Stein & no & no & no \\
\hline
\end{tabular}
All Stein $G$-invariant domains in $G^{\mathbb{C}} / K^{\mathbb{C}}$ are given by the following table.

Table 6.0

Remark. The domains $D_{1}(0)$ and $D_{2}(0)$ are known as Akhiezer-Gindikin domains. They were introduced in [Akhiezer and Gindikin 1990] for $G / K$ of arbitrary rank. In the two-dimensional case, the domains $S_{1}(0)$ and $S_{2}(0)$ are related to the causal structure of the symmetric space $G / H=\mathrm{SO}_{0}(2,1) / \mathrm{SO}(1,1)$. Domains of this type were studied in [Neeb 1999].

Proof. We first show that all the domains listed in the above table are Stein. The Akhiezer-Gindikin domain $D_{1}(0)$ is Stein by [Burns et al. 2003]. For $0<a<1$, the domains $D_{1}(a)$ are $G$-invariant subdomains of $D_{1}(0)$ containing the minimal orbit $G \cdot z_{1} \cong G / K$. Their Steinness follows for example from the nonlinear convexity theorem in [Gindikin and Krötz 2002].

When $G=\mathrm{SO}_{0}(n, 1)$, with $n \geq 2$, the domain $D_{2}(0)$ and its subdomains $D_{2}(a)$ for $0<a<1$ are Stein since they are biholomorphic to $D_{1}(0)$ and $D_{1}(a)$, respectively. One such biholomorphism is given for example by the map

$$
G^{\mathbb{C}} / K^{\mathbb{C}} \rightarrow G^{\mathbb{C}} / K^{\mathbb{C}}, \quad g K^{\mathbb{C}} \mapsto g_{3} g K^{\mathbb{C}},
$$


where $g_{3}=\exp i A_{3}$, with $\alpha\left(A_{3}\right)=\pi / 2$; see (4-2). Note that $g_{3} \in \mathrm{SO}(n, 1) \backslash$ $\left\{\mathrm{SO}_{0}(n, 1)\right\}$; therefore $g_{3} G=G g_{3}$. As a result, the above map exchanges the singular orbits $G \cdot z_{1}$ and $G \cdot z_{3}$ and maps $G \cdot \ell_{1}(a)$ onto $G \cdot \ell_{3}(a)$, for $0<a<1$.

When $G=\mathrm{SO}_{0}(2,1)$, the domains $S_{1}(0)$ and $S_{2}(0)$ and their subdomains $S_{1}(b)$ and $S_{2}(b)$ for $0<b<\infty$ were shown to be Stein in [Neeb 1999].

The last four domains in the list contain in their interior one of the nonclosed orbits $G \cdot w_{i}$ for some $i=1, \ldots, 4$. Their boundary consists of two nonclosed $G$-orbits and the singular orbit in their closure. All of them are Stein if $G=$ $\mathrm{SO}_{0}(2,1) \cong \mathrm{SU}(1,1) /\left\{ \pm I_{2}\right\}$. Only $D_{1}(0) \cup G \cdot w_{1} \cup S_{1}(0)$ and $D_{1}(0) \cup G \cdot w_{4} \cup S_{2}(0)$ are Stein when $G=\mathrm{SU}(n, 1)$ with $n>1$. These facts are proved in Example 6.3 by constructing explicit models of such domains.

To complete the classification, it remains to show that no $G$-invariant domains in $G^{\mathbb{C}} / K^{\mathbb{C}}$ are Stein other than the ones listed in Table 6.0. When $G=\operatorname{SO}_{0}(2,1) \cong$ $\mathrm{SU}(1,1) /\{ \pm \mathrm{Id}\}$ and $G=\mathrm{SU}(n, 1)$ with $n \geq 2$, this is proved in Example 6.3.

In all other cases, namely $\mathrm{SO}_{0}(n, 1)$ with $n>1, \mathrm{Sp}(n, 1)$, and $F_{4}^{*}$, this follows from the description of the $G$-orbit space of $G^{\mathbb{C}} / K^{\mathbb{C}}$ given in diagrams (4-4), (4-9), (4-10) and from the computation of the Levi form of the hypersurface $G$-orbits in $G^{\mathbb{C}} / K^{\mathbb{C}}$. Indeed, by [Geatti 2002, Propositions 5.6 and 5.21], all principal orbits have indefinite Levi form, except for the ones intersecting the slice $\ell_{1}$ (the domain $D_{1}(a)$ is Stein) and, only when the restricted root system of $\mathfrak{g}$ is reduced, the slice $\ell_{3}$ (the domain $D_{2}(a)$ is Stein for $G=\operatorname{SO}_{0}(n, 1)$ ). Moreover, by Remarks 9.10 and 9.18, the Levi form of the nonclosed hypersurface orbits $G \cdot w_{2}$ and $G \cdot w_{5}$ is indefinite. Since the boundary of a Stein domain cannot have indefinite Levi form, the theorem follows.

Let us illustrate the result of Theorem 6.1 on the model of $G^{\mathbb{C}} / K^{\mathbb{C}}$ described in Example 4.4. The Stein, $G$-invariant domains are studied by means of an appropriate $G$-invariant function on $G^{\mathbb{C}} / K^{\mathbb{C}}$.

Example 6.2. Let $G=\mathrm{SO}_{0}(n, 1)$. By Example 4.4, the quotient $G^{\mathbb{C}} / K^{\mathbb{C}}$ can be identified with $M^{\mathbb{C}}:=\left\{\xi \in \mathbb{C}^{n+1}: \xi_{1}^{2}+\cdots+\xi_{n}^{2}-\xi_{n+1}^{2}=-1\right\}$. Assume $n>2$. Consider the $G$-invariant function $f: M^{\mathbb{C}} \rightarrow \mathbb{R}$ defined by

$$
f\left(\xi_{1}, \ldots, \xi_{n+1}\right):=\left|\xi_{1}\right|^{2}+\cdots+\left|\xi_{n}\right|^{2}-\left|\xi_{n+1}\right|^{2}-1 .
$$

For every $0<a<1$, the $G$-invariant domains $D_{1}(a)$ and $D_{2}(a)$ coincide with the two connected components of the set $\left\{\xi \in M^{\mathbb{C}}: f(\xi)<r\right\}$ for some $-2<r<0$. Every such domain is bounded by a single $G$-orbit on which the Levi form of $f$ is positive definite. Hence it is Stein.

The $G$-invariant domains $D_{1}(0)$ and $D_{2}(0)$ coincide with the two connected components of the set $\left\{\xi \in M^{\mathbb{C}}: f(\xi)<0\right\}$. They are bounded by the nonsmooth hypersurfaces $\partial D_{1}(0)=G \cdot\left(z_{2} \cup w_{1}\right)$ and $\partial D_{2}(0)=G \cdot\left(z_{2} \cup w_{2}\right)$, respectively. 
At all smooth points of $\partial D_{1}(0)$ and $\partial D_{2}(0)$, the Levi form of $f$ has $n-2$ positive eigenvalues and one zero eigenvalue. This is consistent with the fact that $D_{1}(0)$ and $D_{2}(0)$ are Stein. The Levi form of $f$ is indefinite on all remaining hypersurface $G$-orbits. Thus there are no other Stein $G$-invariant domains in $M^{\mathbb{C}}$.

Next we determine all Stein, $G$-invariant domains in $G^{\mathbb{C}} / K^{\mathbb{C}}$ in the case $G=$ $\mathrm{SU}(n, 1)$ by using the model of $G^{\mathbb{C}} / K^{\mathbb{C}}$ described in Example 4.7 and Remark 4.8. This settles the missing cases in the proof of Theorem 6.1 .

Example 6.3. Let $G=\operatorname{SU}(n, 1)$ with $n \geq 1$. By Example 4.7, the quotient $G^{\mathbb{C}} / K^{\mathbb{C}}$ can be identified with $M^{\mathbb{C}}:=\mathbb{P}^{n} \times \overline{\mathbb{P}}^{n} \backslash\left\{\langle z, w\rangle_{n, 1}=0\right\}$. Consider the $G$-invariant function $f: M^{\mathbb{C}} \rightarrow \mathbb{R}$ defined by

$$
f([z],[w])=-\frac{\langle z, z\rangle_{n, 1}\langle w, w\rangle_{n, 1}}{\left|\langle z, w\rangle_{n, 1}\right|^{2}} .
$$

Consider first the case $G=\mathrm{SU}(1,1)$.

By computing the Levi form of $f$ on the $G$-orbits in the level set $\{f=r\}$ with $r<0$, one shows that the domains $D_{1}(a)$ and $D_{2}(a)$ are Stein for all $0<a<1$. Similarly one shows that $S_{1}(b)$ and $S_{2}(b)$ are Stein for every $b>0$. One can also verify that the Levi form of $f$ on all nonclosed hypersurface orbits $G \cdot w_{1}, \ldots, G \cdot w_{4}$ is identically zero. This is consistent with the fact that the domains $D_{1}(0), D_{2}(0)$, $S_{1}(0)$ and $S_{2}(0)$ are Stein. We claim that the domains

$$
\begin{array}{ll}
W_{1,1}:=D_{1}(0) \cup G \cdot w_{1} \cup S_{1}(0), & W_{1,2}:=D_{1}(0) \cup G \cdot w_{4} \cup S_{2}(0), \\
W_{2,1}:=D_{2}(0) \cup G \cdot w_{2} \cup S_{1}(0), & W_{2,2}:=D_{1}(0) \cup G \cdot w_{3} \cup S_{2}(0)
\end{array}
$$

are Stein as well. By evaluating the hermitian forms $\langle z, z\rangle_{n, 1}$ and $\langle w, w\rangle_{n, 1}$ on the slices described in Example 4.7 and Remark 4.8, one sees that such domains can be characterized as follows:

$$
\begin{aligned}
& W_{1,1}=\left\{\langle z, w\rangle_{1,1} \neq 0 \text { and }\langle z, z\rangle_{1,1}<0\right\}, \\
& W_{1,2}=\left\{\langle z, w\rangle_{1,1} \neq 0 \text { and }\langle w, w\rangle_{1,1}<0\right\}, \\
& W_{2,1}=\left\{\langle z, w\rangle_{1,1} \neq 0 \text { and }\langle w, w\rangle_{1,1}>0\right\}, \\
& W_{2,2}=\left\{\langle z, w\rangle_{1,1} \neq 0 \text { and }\langle z, z\rangle_{1,1}>0\right\} .
\end{aligned}
$$

As a consequence, the maps defined by

$$
\begin{array}{ll}
\Delta \times \mathbb{C} \rightarrow W_{1,1}, & (u, v) \mapsto([u: 1],[\bar{v}: 1+\bar{u} \bar{v}]), \\
\mathbb{C} \times \Delta \rightarrow W_{1,2}, & (u, v) \mapsto([u: 1+u v],[\bar{v}: 1]), \\
\Delta \times \mathbb{C} \rightarrow W_{2,1}, & (u, v) \mapsto([1+u v: u],[1: \bar{v}]), \\
\mathbb{C} \times \Delta \rightarrow W_{1,2}, & (u, v) \mapsto[1: u],[1+\bar{u} \bar{v}: \bar{v}])
\end{array}
$$


are biholomorphisms. Here $\Delta$ denotes the unit disk in $\mathbb{C}$. In particular the domains $W_{1,1}, \ldots, W_{2,2}$ are Stein, as claimed.

Other $G$-domains in $M^{\mathbb{C}}$ that are possibly Stein can only be obtained as arbitrary unions of domains $W_{k, l}$ for $k, l=1,2$. We claim that such unions are not Stein. For instance, let us show that $W_{1,1} \cup W_{2,1}$ is not Stein. Consider the Stein local chart

$$
\phi: \mathbb{C}^{2} \rightarrow \mathbb{P}^{1} \times \overline{\mathbb{P}}^{1}, \quad(u, v) \mapsto([u: 1],[1: \bar{v}]) .
$$

Since the preimage

$$
\phi^{-1}\left(W_{1,1} \cup W_{2,1}\right)=\left\{(u, v) \in \mathbb{C}^{2}: u \neq v \text { and either }|u|<1 \text { or }|v|<1\right\}
$$

is not Stein, the domain $W_{1,1} \cup W_{2,1}$ is not Stein either. An analogous argument applies to the remaining cases.

Now consider the case $G=\mathrm{SU}(n, 1)$ with $n \geq 1$.

Using the $G$-invariant function $f$, one can prove that the domains $D_{1}(a)$ are Stein for $a>0$. One can also verify that $D_{1}(0)$ coincides with a connected component of the set $\left\{z \in M^{\mathbb{C}} \mid f(z)<0\right\}$ and that on the smooth part of its boundary $\partial D_{1}(0)=G \cdot\left(w_{1} \cup z_{2} \cup w_{4}\right)$, the Levi form of $f$ has nonnegative eigenvalues. This is consistent with the fact that $D_{1}(0)$ is Stein.

Moreover, the Levi form of $f$ is indefinite on the principal $G$-orbits through the slices $\ell_{2}, \ell_{3}, \ell_{4}$, and $\ell_{5}$ and on the nonclosed hypersurface orbit $G \cdot w_{5}$. On the other hand, the Levi form of $f$ is definite on the nonclosed hypersurface orbits $G \cdot w_{2}$ and $G \cdot w_{3}$. As a result, the only other $G$-invariant domains in $M^{\mathbb{C}}$ that are possibly Stein are

$$
W_{1,1}:=D_{1}(0) \cup G \cdot w_{1} \cup S_{1}(0), \quad W_{1,2}:=D_{1}(0) \cup G \cdot w_{4} \cup S_{2}(0), \quad W_{1,1} \cup W_{1,2} .
$$

First we show that $W_{1,1}$ and $W_{1,2}$ are indeed Stein. By evaluating $\langle z, z\rangle_{n, 1}$ and $\langle w, w\rangle_{n, 1}$ on the slices described in Example 4.7, one sees that such domains can be characterized as follows:

$$
\begin{aligned}
& W_{1,1}=\left\{([z],[w]) \in \mathbb{P}^{n} \times \overline{\mathbb{P}}^{n}:\langle z, w\rangle_{n, 1} \neq 0 \text { and }\langle z, z\rangle_{n, 1}<0\right\}, \\
& W_{1,2}=\left\{([z],[w]) \in \mathbb{P}^{n} \times \overline{\mathbb{P}}^{n}:\langle z, w\rangle_{n, 1} \neq 0 \text { and }\langle w, w\rangle_{n, 1}<0\right\} .
\end{aligned}
$$

As a consequence the maps

$$
\begin{array}{ll}
\mathbb{B}^{n} \times \mathbb{C}^{n} \rightarrow W_{1,1}, & (u, v) \mapsto\left([u: 1],\left[v: 1+\bar{u}_{1} \bar{v}_{1}+\cdots+\bar{u}_{n} \bar{v}_{n}\right]\right), \\
\mathbb{C}^{n} \times \mathbb{B}^{n} \rightarrow W_{1,2}, & (u, v) \mapsto\left(\left[u: 1+u_{1} v_{1}+\cdots+u_{n} v_{n}\right],[\bar{v}: 1]\right)
\end{array}
$$

are biholomorphisms. Here $\mathbb{B}^{n}$ denotes the unit ball in $\mathbb{C}^{n}$. In particular $W_{1,1}$ and $W_{1,2}$ are Stein, as claimed. 
Next we show the domain $\Omega:=W_{1,1} \cup W_{1,2}$ with $\partial \Omega=G \cdot\left(w_{2} \cup z_{2} \cup w_{3}\right)$ is not Stein. Assume by contradiction that $\Omega$ is Stein. Let $\mathfrak{c}^{\prime}$ be the abelian subalgebra generating the Cartan subset $\mathscr{C}^{\prime}$ (see Example 4.7). Let $T=\exp \mathfrak{c}^{\prime}$ be the corresponding compact torus in $G$. Consider the $T$-action on $\Omega$ and the induced local holomorphic $T^{\mathbb{C}}$-action. By the globalization theorem in [Heinzner 1991, Section 6.6], the domain $\Omega$ embeds in its $T^{\mathbb{C}}$-globalization $\Omega^{*}$ as a $T$-invariant, orbit-convex subset. By definition, this means that the intersection of $\Omega$ with an $\exp i$ c-orbit in $\Omega^{*}$ is connected.

Every $T^{\mathbb{C}}$-orbit through the slice $\ell_{1}$ is contained in $\Omega$. Indeed in $M^{\mathbb{C}}$ one can verify that

$$
\begin{aligned}
\exp \left(i s C^{\prime}\right) \cdot \ell_{1}(t)=\left(\left[0: \ldots: e^{s} i \sin \frac{\pi}{4}(1-t): e^{-s} \cos \frac{\pi}{4}(1-t)\right]\right. & \\
& {\left.\left[0: \ldots:-e^{-s} i \sin \frac{\pi}{4}(1-t): e^{s} \cos \frac{\pi}{4}(1-t)\right]\right) . }
\end{aligned}
$$

Thus for fixed $0<t<1$, the function $\mathbb{R} \rightarrow \mathbb{R}$ defined by $s \mapsto f\left(\exp \left(i s C^{\prime}\right) \cdot \ell_{1}(t)\right)$ is given by

$$
\left(e^{2 s} \sin ^{2} \frac{\pi}{4}(1-t)-e^{-2 s} \cos ^{2} \frac{\pi}{4}(1-t)\right)\left(e^{-2 s} \sin ^{2} \frac{\pi}{4}(1-t)-e^{2 s} \cos ^{2} \frac{\pi}{4}(1-t)\right)
$$

and vanishes exactly twice, namely on $G \cdot w_{1}$ and on $G \cdot w_{4}$. Therefore $\exp \left(i \mathfrak{c}^{\prime}\right) \cdot \ell_{1}(t)$ never crosses the boundary of $\Omega$ and consequently the complex orbit $T^{\mathbb{C}} \cdot \ell_{1}(t)$ is entirely contained in $\Omega$, as claimed. Moreover, for every fixed $s>0$, one has

$$
\begin{gathered}
\lim _{n \rightarrow \infty} \exp \left(i s C^{\prime}\right) \cdot \ell_{1}(1 / n)=\ell_{2}(s) \in \Omega, \\
\lim _{n \rightarrow \infty} \exp \left(-i s C^{\prime}\right) \cdot \ell_{1}(1 / n)=\ell_{4}(s) \in \Omega .
\end{gathered}
$$

Then the orbit-convexity of $\Omega$ in $\Omega^{*}$ implies that the sequence $\left\{\ell_{1}(1 / n)\right\}_{n}$ has a limit point in $\Omega$. On the other hand, in $G^{\mathbb{C}} / K^{\mathbb{C}}$ one has $\lim _{n} \ell_{1}(1 / n)=z_{2}$, which is not in $\Omega$. This yields a contradiction and proves that $\Omega$ is not Stein. The classification of all Stein $G$-invariant domains in $M^{\mathbb{C}}$ is now complete.

We conclude this section with a remark which is a consequence of Theorem 6.1 and is often used in the sequel.

Remark 6.4. Let $D$ be a domain in $G^{\mathbb{C}} / K^{\mathbb{C}}$ with smooth boundary $\partial D$. It is well known that if $D$ is not pseudoconvex at $z \in \partial D$, then no holomorphic function on $D$ diverges in the vicinity of $z$. Let $\ell: I \rightarrow G^{\mathbb{C}} / K^{\mathbb{C}}$ be a slice for principal $G$-orbits in $G^{\mathbb{C}} / K^{\mathbb{C}}$. By the classification of Stein, $G$-invariant domains in $G^{\mathbb{C}} / K^{\mathbb{C}}$ given in Theorem 6.1, the following facts hold.

(i) Assume that the Levi form of the orbits parametrized by $\ell$ is definite. Let $(c, d) \subset I$ be an interval with $0 \leq c<d$ and $d \in I$. Then no holomorphic function on the invariant domain $G \cdot \ell((c, d))$ diverges in the vicinity of the boundary orbit $G \cdot \ell(d)$ (for instance, if $I=(0,1)$ and $l=l_{1}$, then the domain 
$D_{1}(d)$ is strictly pseudoconvex at every point of the boundary orbit $G \cdot \ell_{1}(d)$. Thus the domain $G \cdot \ell_{1}((c, d))$ is not pseudoconvex at any point of $\left.G \cdot \ell_{1}(d)\right)$.

(ii) Assume that the Levi form of the orbits parametrized by $\ell$ is indefinite. Let $(c, d) \subset I$ be an interval with $c \in I$. Then no holomorphic function on the invariant domain $G \cdot \ell((c, d))$ diverges in the vicinity of the boundary orbit $G \cdot \ell(c)$. Similarly, if $d \in I$, then no holomorphic function diverges in the vicinity of $G \cdot \ell(d)$.

\section{Univalence over $G^{\mathbb{C}} / K^{\mathbb{C}}$}

Let $G$ be a connected, noncompact, real simple Lie group, let $K \subset G$ be a maximal compact subgroup, and let $G^{\mathbb{C}}$ be the universal complexification of $G$. Assume that the center $\Gamma$ of $G$ is finite and that $G$ is not a covering of $\operatorname{SL}(2, \mathbb{R})$. In this section, we show that a holomorphically separable, $G$-equivariant Riemann domain $q: \Sigma \rightarrow G^{\mathbb{C}} / K^{C}$ is necessarily univalent if the rank of $G / K$ is equal to one; see Theorem 7.6 and Remark 7.8.

In most cases the map $q$ is injective on every $G$-orbit; see Section 5. So we are reduced to prove the injectivity of $q$ over the global slices for the $G$-action defined by diagrams (4-3), (4-4), (4-9), and (4-10). Recall that the slices parametrizing principal $G$-orbits are diffeomorphic to open intervals of $\mathbb{R}$ and that a local diffeomorphism of a one-dimensional smooth manifold into the real line $\mathbb{R}$ is necessarily injective. As a consequence, $q$ is injective on every connected component of $\Sigma$ over a domain in $G^{\mathbb{C}} / K^{\mathbb{C}}$ consisting of principal orbits.

However, in order to ensure monodromy around the singular orbit $G \cdot z_{2}$ (see the diagrams in Section 4), it is necessary to combine the uniqueness property of path liftings for Riemann domains with the complex geometry of the $G$-invariant domains in $G^{\mathbb{C}} / K^{\mathbb{C}}$. Before proving the main result of this section, some preliminary lemmas are needed.

Let $\ell: I \rightarrow G^{\mathbb{C}} / K^{\mathbb{C}}$ be one of the slices for principal $G$-orbits defined in (4-5), (4-6), (4-11), (4-12) and (4-13). Define

$$
\hat{I}:= \begin{cases}(0,1] & \text { if } I=(0,1) \\ I & \text { if } I=\mathbb{R}^{>0}\end{cases}
$$

Recall that $I=(0,1)$ only when $\ell=\ell_{1}$ or $\ell=\ell_{3}$. In those cases extend $\ell$ to $\hat{I}=(0,1]$ by defining

$$
\ell_{1}(1):=e K^{\mathbb{C}} \quad \text { and } \quad \ell_{3}(1):=\exp \left(i A_{3}\right) K^{\mathbb{C}} .
$$

We refer to $\ell: \hat{I} \rightarrow G^{\mathbb{C}} / K^{\mathbb{C}}$ as an extended slice. Note that the images of the extended slices $\ell_{1}$ and $\ell_{3}$ include the points $z_{1}$ and $z_{3}$, respectively. 
Let $q: \Sigma \rightarrow G^{\mathbb{C}} / K^{\mathbb{C}}$ be a $G$-equivariant Riemann domain, and let $\ell: \hat{I} \rightarrow G^{\mathbb{C}} / K^{\mathbb{C}}$ be an extended slice. A local lifting of $\ell$ is a smooth path $\tilde{\ell}: J \rightarrow \Sigma$ defined on a nonempty interval $J$ open in $\hat{I}$, and satisfying the condition $q \circ \tilde{\ell}=\ell$ on $J$. A local lifting $\tilde{\ell}: J \rightarrow \Sigma$ is maximal if it cannot be extended to a larger interval $J^{\prime}$ with $J \varsubsetneqq J^{\prime} \subset \hat{I}$.

Lemma 7.1. Assume that $G$ is embedded in its universal complexification $G^{\mathbb{C}}$ and is different from $\operatorname{SL}(2, \mathbb{R})$ and $\operatorname{Spin}(3,1)$. Let $q: \Sigma \rightarrow G^{\mathbb{C}} / K^{\mathbb{C}}$ be a Stein, $G$ equivariant Riemann domain, and let $\tilde{\ell}: J \rightarrow \Sigma$ be a maximal local lifting of an extended slice $\ell: \hat{I} \rightarrow G^{\mathbb{C}} / K^{\mathbb{C}}$.

(i) if the Levi form of the principal orbits parametrized by $\ell$ is definite, then the invariant domain $G \cdot \ell(J)$ in $G^{\mathbb{C}} / K^{\mathbb{C}}$ is Stein; see Theorem 6.1.

(ii) If the Levi form of the principal orbits parametrized by $\ell$ is indefinite, then $J$ coincides with $\hat{I}$.

Proof. (i) Consider first the case $\hat{I}=\mathbb{R}^{>0}$ ( see diagram (4-3), Example 6.3 and Remark 4.8$)$. By Theorem 6.1, we need to show that $J=(b,+\infty)$ for some $b \geq 0$. Assume by contradiction that $J=(b, d)$ with $0 \leq b<d<\infty$. Since the lifting $\tilde{\ell}(J)$ is a one-dimensional real-analytic submanifold of $\Sigma$, the local diffeomorphism $q \mid \tilde{\ell}(J)$ is injective. By Proposition 5.7, the map $q$ is injective on every $G$-orbit. Therefore the restriction $q \mid G \cdot \tilde{\ell}(J): G \cdot \tilde{\ell}(J) \rightarrow G \cdot \ell(J)$ is a biholomorphism.

By the maximality of $\tilde{\ell}$, when $n$ grows, the sequence $\{\tilde{\ell}(d-1 / n)\}_{n}$ leaves every given compact subset in $\Sigma$. Since $\Sigma$ is Stein, there exists a holomorphic function $f \in \mathcal{O}(\Sigma)$ such that $\lim _{n \rightarrow \infty}|f(\tilde{\ell}(d-1 / n))|=\infty$.

On the other hand, the push-forward of $f$ by $q \mid G \cdot \tilde{\ell}(J)$ defines a holomorphic function in $O(G \cdot \ell(J))$ that diverges in the vicinity of the boundary orbit $G \cdot \ell(d)$. This contradicts Remark 6.4(ii), implying that $J$ is of the form $(b, \infty)$, as claimed.

Consider now the case $\hat{I}=(0,1]$. This only occurs for $\ell=\ell_{1}$ or, when the restricted root system of $\mathfrak{g}$ is reduced, for $\ell=\ell_{3}$; see the diagrams in Section 4 and [Geatti 2002, Proposition 5.6]. By Theorem 6.1, we need to show that $J=(a, 1]$ for some $a \geq 0$. Assume by contradiction that $J=(a, d)$ with $0 \leq a<d \leq 1$. The argument used in the previous case shows that $J=(a, 1)$ and that there exists a holomorphic function $f \in \mathcal{O}(\Sigma)$ such that $\lim _{n \rightarrow \infty}|f(\tilde{\ell}(1-1 / n))|=\infty$. Moreover, the push-forward of $f$ by $q \mid G \cdot \tilde{\ell}(J)$ defines a holomorphic function $\bar{f} \in \mathcal{O}(G \cdot \ell(J))$, which diverges in the vicinity of the boundary orbit $G \cdot \ell(1)$. On the other hand, such an orbit is a totally real submanifold of $G \cdot \ell((a, 1])$. Thus $\bar{f}$ extends to a holomorphic function on $G \cdot \ell((a, 1])$. This yields a contradiction, implying that $J=(a, 1]$, as desired.

(ii) Assume first that $\hat{I}=\mathbb{R}^{>0}$. Then Remark 6.4(ii) and an argument analogous to the proof of (i) show that $J=\hat{I}$. Consider then the case $\hat{I}=(0,1]$. This only occurs 
when the restricted root system of $\mathfrak{g}$ is nonreduced and $\ell=\ell_{3}$; see the diagrams in Section 4 and [Geatti 2002, Proposition 5.6]. An argument like the proof of (i) shows that if a lifting $\tilde{\ell}_{3}: J \rightarrow \Sigma$ is maximal, then either $J=(0,1]$ or $J=(0,1)$.

To prove that $J=(0,1]$, suppose by contradiction that $J=(0,1)$. Consider a sequence $\left\{z_{n}\right\}$ in $G \cdot \ell_{3}(J)$ that converges to a point on the boundary orbit $G \cdot w_{5}$, say $w_{5}$. Since the Levi form of $G \cdot w_{5}$ is indefinite (see Remark 9.10), no holomorphic function on $G \cdot \ell_{3}(J)$ diverges on $\left\{z_{n}\right\}$. Note that the restriction

$$
q \mid G \cdot \tilde{\ell}_{3}(J): G \cdot \tilde{\ell}_{3}(J) \rightarrow G \cdot \ell_{3}(J)
$$

is a biholomorphism. Hence no holomorphic function of $G \cdot \tilde{\ell}_{3}(J)$ diverges on the sequence $\left\{\zeta_{n}\right\}$ in $\Sigma$ defined by $\zeta_{n}:=\left(q \mid G \cdot \tilde{\ell}_{3}(J)\right)^{-1}\left(z_{n}\right)$. By the Steinness of $\Sigma$, there exists a subsequence of $\left\{\zeta_{n}\right\}$ converging to a point $\eta_{5}$ in $\Sigma$. Since $q$ is continuous, one has $q\left(\eta_{5}\right)=w_{5}$.

By the $G$-equivariance of $q$, the description of the slice representation at $z_{3}$ given in Remark 4.2, and Proposition 5.7, there exists a $G$-invariant neighborhood $V$ of $\eta_{5}$ in $\Sigma$ on which $q$ is injective. Its image $q(V)$ intersects the slice $\ell_{5}$ in $\ell_{5}((0, \epsilon))$ for some $\epsilon>0$. By statement (i) of this lemma, the local lifting $s \mapsto(q \mid V)^{-1}\left(\ell_{5}(s)\right)$, with $s \in(0, \epsilon)$, extends to a lifting $\tilde{\ell}_{5}: \hat{I}_{5} \rightarrow \Sigma$ of $\ell_{5}$. Note that $q$ maps the $G$-invariant domain $W:=G \cdot\left(\tilde{\ell}_{3}(J) \cup \eta_{5} \cup \tilde{\ell}_{5}\left(\hat{I}_{5}\right)\right)$ in $\Sigma$ biholomorphically onto the domain $q(W)=G \cdot\left(\ell_{3}(J) \cup w_{5} \cup \ell_{5}\left(\hat{I}_{5}\right)\right)$ in $G^{\mathbb{C}} / K^{\mathbb{C}}$. Since $G \cdot \ell_{3}(1)$ is a totally real submanifold of $q(W) \cup G \cdot \ell_{3}(1)$ (see [Geatti 2002, Lemma 2.11 and Remark 2.13]), every holomorphic function on $q(W)$ extends to a holomorphic function on $q(W) \cup G \cdot \ell_{3}(1)$. As a consequence, no holomorphic function on $W$ can diverge on the sequence $\left\{\tilde{\ell}_{3}(1-1 / n)\right\}_{n}$ in $\Sigma$.

On the other hand, by the maximality of $\tilde{\ell}_{3}$, the sequence $\{\tilde{\ell}(1-1 / n)\}_{n}$ leaves every given compact subset in $\Sigma$ as $n$ grows. Since $\Sigma$ is Stein, there exists a holomorphic function $f \in \mathcal{O}(\Sigma)$ such that $\lim _{n \rightarrow \infty}\left|f\left(\tilde{\ell}_{3}(b-1 / n)\right)\right|=\infty$. This yields a contradiction, implying that $J$ necessarily coincides with $(0,1]$.

Let $\ell_{1}$ and $\ell_{3}$ be the slices parametrizing the principal orbits through the fundamental Cartan subset $\mathscr{A}$. Denote by $\mathscr{C}=\exp i \mathfrak{c} \cdot z_{2}$ the standard Cartan subspace with base point $z_{2}$, and define $\mathscr{C}^{*}:=\mathscr{C} \backslash\left\{z_{2}\right\}$. Recall that in the reduced case, $\mathfrak{c}=\mathbb{R}(X+\theta(X))$ for some nonzero vector $X \in \mathfrak{g}^{\alpha}$, and $z_{2}=\exp \left(i A_{2}\right) K^{\mathbb{C}}$ with $\alpha\left(A_{2}\right)=\pi / 2$. In the nonreduced case, $\mathfrak{c}=\mathbb{R}(X+\theta(X))$ for some nonzero vector $X \in \mathfrak{g}^{2 \alpha}$, and $z_{2}=\exp \left(i A_{2}\right) K^{\mathbb{C}}$ with $\alpha\left(A_{2}\right)=\pi / 4$. In both cases, exp $\mathfrak{c}$ is a compact, one-dimensional, real torus in $G$, which we denote by $T$. Both $T$ and its universal complexification $T^{\mathbb{C}} \cong \mathbb{C}^{*}$ act on $G^{\mathbb{C}} / K^{\mathbb{C}}$ by left translations.

In the next proposition, we single out two distinguished $G$-invariant domains $\Omega$ and $\Omega^{\prime}$ in $G^{\mathbb{C}} / K^{\mathbb{C}}$ containing all $T^{\mathbb{C}}$-orbits through the slices $\ell_{1}\left(I_{1}\right)$ and $\ell_{3}\left(I_{3}\right)$, respectively. 
Lemma 7.2. Let $G / K$ be a noncompact, rank-one, Riemannian symmetric space. Consider the domain in $G^{\mathbb{C}} / K^{\mathbb{C}}$ defined by

$$
\Omega:=G \cdot\left(\ell_{1}\left(I_{1}\right) \cup w_{1} \cup w_{4} \cup \mathscr{C}^{*}\right) .
$$

Then for every point $z \in \ell_{1}\left(I_{1}\right)$, the complex orbit $T^{\mathbb{C}} \cdot z$ is contained in $\Omega$.

Similarly, define

$$
\Omega^{\prime}:=G \cdot\left(\ell_{3}\left(I_{3}\right) \cup w_{2} \cup w_{3} \cup \mathscr{C}^{*}\right) .
$$

Then for every point $z \in \ell_{3}\left(I_{3}\right)$, the complex orbit $T^{\mathbb{C}} \cdot z$ is contained in $\Omega^{\prime}$.

Proof. We first assume that $G=\mathrm{SO}_{0}(2,1)$ and prove the statement by using the model $M^{\mathbb{C}}$ of $G^{\mathbb{C}} / K^{\mathbb{C}}$ constructed in Example 4.4. Let $C$ be the generator of $\mathfrak{c}$ chosen there. Then, for $s \in \mathbb{R}$ and $t \in(0,1)$, one has

$$
\exp (i s C) \cdot \ell_{1}(t)=\left(\sinh (2 s) \sin \frac{\pi}{2}(1-t), i \cosh (2 s) \sin \frac{\pi}{2}(1-t), \cos \frac{\pi}{2}(1-t)\right) .
$$

Since $z_{2}=(0, i, 0)$ and the entries of the matrix group $G$ are real, from the above expression one easily verifies that $\exp i \mathfrak{c} \cdot \ell_{1}\left(I_{1}\right) \cap G \cdot z_{2}=\varnothing$. Consider then the $G$-invariant function $f(z)=\left|z_{1}\right|^{2}+\left|z_{2}\right|^{2}-\left|z_{3}\right|^{2}-1$ defined on $M^{\mathbb{C}}$. The function $f$ vanishes on the real hypersurface $G \cdot\left(z_{2} \bigcup_{j=1}^{4} w_{j}\right)$, is negative on the sets $G \cdot \ell_{j}\left(I_{j}\right)$ for $j=1,3$, and is positive on the sets $G \cdot \ell_{j}\left(I_{j}\right)$ for $j=2$, 4. Moreover, for every fixed $t_{0} \in(0,1)$, one sees that

$$
f\left(\exp (i s C) \cdot \ell_{1}\left(t_{0}\right)\right)=\left(\sinh ^{2} 2 s+\cosh ^{2} 2 s\right) \sin ^{2} \frac{\pi}{2}\left(1-t_{0}\right)-\cos ^{2} \frac{\pi}{2}\left(1-t_{0}\right)-1
$$

is strictly increasing as $|s| \rightarrow \infty$. Thus it vanishes exactly twice. As a consequence, the path $\exp (i s C) \cdot \ell_{1}\left(t_{0}\right)$ crosses the hypersurface $f^{-1}(0) \backslash\left\{G \cdot z_{2}\right\}$ exactly twice, namely on the orbits $G \cdot w_{1}$ and $G \cdot w_{2}$. It follows that $\exp ($ is $C) \cdot \ell_{1}\left(t_{0}\right) \in \Omega$, for every $s \in \mathbb{R}$. Thus the $T^{\mathbb{C}}$-orbit through $\ell_{1}\left(t_{0}\right)$ is entirely contained in $\Omega$, as stated. An analogous argument proves the statement for the higher dimensional hyperquadrics. By Remark 4.1(ii), this settles the case when $\mathfrak{g}$ has a reduced restricted root system.

Consider now the case when the restricted root system of $\mathfrak{g}$ is nonreduced. We prove the statement by reducing to the two-dimensional case. Set $\hat{\mathfrak{g}}:=\mathfrak{s o}(2,1)$ and fix a basis in $\hat{\mathfrak{g}}$ of the form $\{\widehat{X}, \theta(\widehat{X}), \hat{A}=[\theta(\widehat{X}), \widehat{X}]\}$, where $\widehat{X}$ is a root vector in $\hat{\mathfrak{g}}^{\alpha}$ and $\alpha(\hat{A})=\pi / 2$. Define $\widehat{C}=\widehat{X}+\theta(\widehat{X})$. Choose a root vector $X \in \mathfrak{g}^{2 \alpha}$ and normalize the triple $\{X, \theta(X), A=[\theta(X), X]\}$ in $\mathfrak{g}$ so that $\alpha(A)=\pi / 4$. Such a triple generates a three-dimensional $\theta$-stable subalgebra of $\mathfrak{g}$ isomorphic to $\hat{\mathfrak{g}}$. In particular, there exists an injective Lie algebra homomorphism $\varphi_{*}: \hat{\mathfrak{g}} \rightarrow \mathfrak{g}$ mapping $\widehat{X}, \hat{A}$, and $\theta(\widehat{X})$ into $X, A$, and $\theta(X)$, respectively. Clearly $\varphi_{*}$ maps $\widehat{C}=\widehat{X}+\theta(\widehat{X})$ into $C=X+\theta(X)$ as well. Let $\widehat{K}=\mathrm{SO}(2)$ be the maximal compact subgroup of $\widehat{G}:=\operatorname{SO}_{0}(2,1)$, and let $\hat{\mathfrak{k}}$ be its Lie algebra. Note that $\hat{\mathfrak{k}}$ and $\mathfrak{k}$ are generated by $\widehat{C}$ and 
$C$, respectively. One can check that the $\mathbb{C}$-linear extension $\hat{\mathfrak{g}}^{\mathbb{C}} \rightarrow \mathfrak{g}^{\mathbb{C}}$ of $\varphi_{*}$ induces a Lie group morphism $\varphi: \widehat{G}^{\mathbb{C}} \rightarrow G^{\mathbb{C}}$ mapping $\widehat{K}^{\mathbb{C}}$ to $K^{\mathbb{C}}$. As a consequence, one obtains a holomorphic map (denoted by the same symbol) $\varphi: \widehat{G}^{\mathbb{C}} / \widehat{K}^{\mathbb{C}} \rightarrow G^{\mathbb{C}} / K^{\mathbb{C}}$.

Let $\widehat{\Omega}$ be the domain

$$
\widehat{\Omega}=\widehat{G} \cdot\left(\hat{\ell}_{1}\left(I_{1}\right) \cup \hat{w}_{1} \cup \hat{\ell}_{2}\left(I_{2}\right) \cup \hat{w}_{4} \cup \hat{\ell}_{4}\left(I_{4}\right)\right)
$$

in $\widehat{G}^{\mathbb{C}} / \widehat{K}^{\mathbb{C}}$, for which the statement has been proved above. We claim that $\varphi(\widehat{\Omega}) \subset$ $\Omega$. The map $\varphi$ is "equivariant" with respect to the action of $\widehat{G}$, that is $\varphi(g \cdot x)=$ $\varphi(g) \cdot \varphi(x)$ for every $g \in \widehat{G}$ and $x \in \widehat{G}^{\mathbb{C}} / \widehat{K}^{\mathbb{C}}$. By the definition of $\varphi_{*}$, one has $\varphi(\exp (i t \hat{A}))=\exp (i t A)$ and $\varphi(\exp ($ it $\widehat{C}))=\exp ($ it $C)$. It follows that

$$
\varphi\left(\hat{\ell}_{1}\left(I_{1}\right)\right)=\ell_{1}\left(I_{1}\right), \quad \varphi\left(\hat{z}_{2}\right)=z_{2}, \quad \varphi(\widehat{\mathscr{C}})=\mathscr{C} .
$$

We finish proving the claim by showing that $\varphi\left(\hat{w}_{1}\right) \in G \cdot w_{1}$ and $\varphi\left(\hat{w}_{4}\right) \in G \cdot w_{4}$ (possibly the orbit $G \cdot w_{4}$ and $G \cdot w_{1}$ coincide). By (4-1), there is a commutative diagram

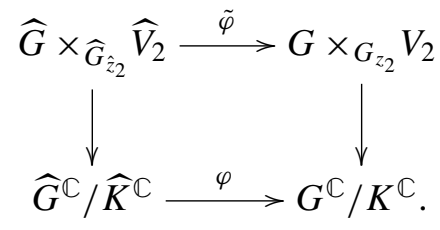

The vertical arrows correspond to the equivariant embeddings given in (4-1), and the map $\tilde{\varphi}$ is defined by $[\hat{g}, \widehat{X}] \rightarrow\left[\varphi(\hat{g}), \varphi_{*}(\widehat{X})\right]$. Since $\varphi_{*}$ is an injective homomorphism, $\varphi\left(\hat{w}_{1}\right)$ does not lie on the singular orbit $G \cdot z_{2}$. Indeed in the twisted bundle $G \times_{G_{z 2}} V_{2}$ such an orbit corresponds to the set $\{[g, 0]: g \in G\}$. On the other hand, $\varphi\left(\hat{w}_{1}\right) \in \overline{G \cdot \ell_{1}\left(I_{1}\right)} \cap \overline{G \cdot \ell_{2}\left(I_{2}\right)}$. Therefore the image $\varphi\left(\hat{w}_{1}\right)$ necessarily lies on the orbit $G \cdot w_{1}$. Similarly one proves that $\varphi\left(\hat{w}_{4}\right) \in G \cdot w_{4}$. In conclusion, $\widehat{\Omega}$ is mapped by $\varphi$ into $\Omega$, as claimed.

Note that $\exp \mathfrak{c}^{\mathbb{C}} \cdot \ell_{1}\left(I_{1}\right)=\varphi\left(\exp \hat{\mathfrak{c}}^{\mathbb{C}} \cdot \hat{\ell}_{1}\left(I_{1}\right)\right)$, and recall that in the 2-dimensional case we already showed that $\exp \hat{\mathfrak{c}}^{\mathbb{C}} \cdot \hat{\ell}_{1}\left(I_{1}\right) \subset \widehat{\Omega}$. Then, by the above claim, one has $T^{\mathbb{C}} \cdot \ell_{1}(z) \subset \Omega$ for every $z \in \ell_{1}\left(I_{1}\right)$, as required. The statement regarding the domain $\Omega^{\prime}$ follows from similar arguments.

Lemma 7.3. Assume that $G$ is embedded in its universal complexification $G^{\mathbb{C}}$ and is different from the groups $\operatorname{SL}(2, \mathbb{R})$ and $\operatorname{Spin}(3,1)$. Let $q: \Sigma \rightarrow G^{\mathbb{C}} / K^{\mathbb{C}}$ be a Stein, G-equivariant Riemann domain.

(i) Let $\tilde{\ell}_{1}: I_{1} \rightarrow \Sigma$ be a lifting of the slice $\ell_{1}$. Assume that the closure of $G \cdot \tilde{\ell}_{1}\left(I_{1}\right)$ in $\Sigma$ contains points $\eta_{1}$ and $\eta_{4}$ mapped by $q$ into the nonclosed orbits $G \cdot w_{1}$ and $G \cdot w_{4}$, respectively (possibly the orbits $G \cdot w_{1}$ and $G \cdot w_{4}$ coincide). Then the singular orbit $G \cdot z_{2}$ is contained in $q(\Sigma)$. 
(ii) Let $\tilde{\ell}_{3}: I_{3} \rightarrow \Sigma$ be a lifting of the slice $\ell_{3}$. Assume that the closure of $G \cdot \tilde{\ell}_{3}\left(I_{3}\right)$ in $\Sigma$ contains points $\eta_{2}$ and $\eta_{3}$ mapped by $q$ into the nonclosed orbits $G \cdot w_{2}$ and $G \cdot w_{3}$, respectively (possibly the orbits $G \cdot w_{2}$ and $G \cdot w_{3}$ coincide). Then the singular orbit $G \cdot z_{2}$ is contained in $q(\Sigma)$.

Proof. (i) We begin by showing that $\Sigma$ contains an open $G$-invariant set that is biholomorphic to the domain $\Omega=G \cdot\left(\ell_{1}\left(I_{1}\right) \cup w_{1} \cup w_{4} \cup \mathscr{C}^{*}\right)$ of Lemma 7.2. By the $G$-equivariance of $q$, by the description of the slice representation at $z_{2}$ given in Remark 4.2, and by Proposition 5.7, there exists a $G$-invariant neighborhood $V$ of $\eta_{1}$ in $\Sigma$ on which $q$ is injective. Its image $q(V)$ intersects the slice $\ell_{2}$ in $\ell_{2}((0, \epsilon))$ for some $\epsilon>0$. By Lemma 7.1(i), the map $s \mapsto(q \mid V)^{-1}\left(\ell_{2}(s)\right)$, with $s \in(0, \epsilon)$, extends to a lifting $\tilde{\ell}_{2}: I_{2} \rightarrow \Sigma$ of $\ell_{2}$. A similar argument yields a lifting $\tilde{\ell}_{4}: I_{4} \rightarrow \Sigma$ of $\ell_{4}$. Since $q$ is injective on the set $\tilde{\ell}_{1}\left(I_{1}\right) \cup \eta_{1} \cup \tilde{\ell}_{2}\left(I_{2}\right) \cup \eta_{4} \cup \tilde{\ell}_{4}\left(I_{4}\right)$, as well as on every $G$-orbit (see Proposition 5.7), it is injective on the $G$-invariant subdomain of $\Sigma$ given by

$$
W:=G \cdot\left(\tilde{\ell}_{1}\left(I_{1}\right) \cup \eta_{1} \cup \tilde{\ell}_{2}\left(I_{2}\right) \cup \eta_{4} \cup \tilde{\ell}_{4}\left(I_{4}\right)\right) .
$$

Note that $q(W)=\Omega$. In particular $W$ is biholomorphic to $\Omega$, as claimed.

Let $\mathscr{C}=\exp i \mathfrak{c} \cdot z_{2}$ be the standard Cartan subset in $G^{\mathbb{C}} / K^{\mathbb{C}}$ starting at $z_{2}$. Recall that $T:=\exp \mathfrak{c}$ is a compact torus in $G$. By Heinzner's globalization theorem [1991, Section 6.6], the space $\Sigma$ can be embedded in its $T^{\mathbb{C}}$-globalization $\Sigma^{*}$ as a $T$-invariant, orbit-convex domain. By definition, this means that the intersection of $\Sigma$ with an exp $i \mathfrak{c}$-orbit in $\Sigma^{*}$ is necessarily connected.

Consider now the induced local $T^{\mathbb{C}}$-orbit of a point $\zeta \in \tilde{\ell}_{1}\left(I_{1}\right)$ in $\Sigma$. Since $q \mid W$ is biholomorphic and $G$-equivariant by Lemma 7.2, such an orbit is in fact global. Let $C$ be a generator of the abelian subalgebra $c$. For every fixed $s>0$, one has

$$
\begin{gathered}
\lim _{n \rightarrow \infty} \exp (i s C) \cdot \tilde{\ell}_{1}(1 / n)=\tilde{\ell}_{2}(s) \in W, \\
\lim _{n \rightarrow \infty} \exp (-i s C) \cdot \tilde{\ell}_{1}(1 / n)=\tilde{\ell}_{4}(s) \in W .
\end{gathered}
$$

By the orbit-convexity of $\Sigma$ in its $T^{\mathbb{C}}$-globalization, the sequence $\left\{\tilde{\ell}_{1}(1 / n)\right\}_{n}$ converges to a point $\zeta_{2} \in \Sigma$. By the continuity of $q$, one has $q\left(\zeta_{2}\right)=z_{2}$. Therefore $z_{2} \in q(\Sigma)$, as required.

Part (ii) is proved by showing that $\Sigma$ contains an open subset biholomorphic to the domain $\Omega^{\prime}$ of Lemma 7.2 and arguing as in the previous case.

Let $G$ be a connected Lie group and $\widetilde{G} \rightarrow G=\widetilde{G} / \Gamma$ a covering of $G$. If $X$ is a $G$-manifold, it can be regarded as a $\widetilde{G}$-manifold by letting $\Gamma$ act trivially on it.

Lemma 7.4. Let $G$ be a connected, real Lie group, and let $\widetilde{G} \rightarrow G=\widetilde{G} / \Gamma$ be a finite covering of $G$. Let $X$ be a complex $G$-manifold with the property that every 
Stein, G-equivariant Riemann domain over $X$ is univalent. Let $q: \Sigma \rightarrow X$ be a Stein, $\widetilde{G}$-equivariant Riemann domain. Then

(i) the image $q(\Sigma)$ is biholomorphic to the quotient $\Sigma / \Gamma$, and $q: \Sigma \rightarrow q(\Sigma)$ can be identified with the quotient map;

(ii) $q$ is a $\widetilde{G}$-equivariant covering.

In particular, $q(\Sigma)$ is Stein.

Proof. (i) Since $\Gamma$ is a finite subgroup of $\widetilde{G}$, the quotient $\Sigma / \Gamma$ can be regarded as the categorical quotient of $\Sigma$ with respect to $\Gamma$. Then $\Sigma / \Gamma$ is a Stein space, and the quotient map $\pi: \Sigma \rightarrow \Sigma / \Gamma$ is holomorphic; see Theorem 2.1. Moreover, since $q$ is $\Gamma$-invariant, there exists a $G$-equivariant holomorphic map $\hat{q}: \Sigma / \Gamma \rightarrow X$ making the diagram

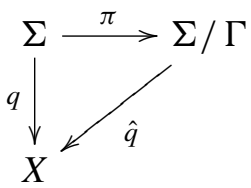

commute. Since $q=\hat{q} \circ \pi$ is locally biholomorphic, then $\pi$ is also locally biholomorphic. In particular, $\Sigma / \Gamma$ is a manifold and $\hat{q}: \Sigma / \Gamma \rightarrow X$ is a Stein, $G$-equivariant Riemann domain. By the assumption on $X$, the map $\hat{q}$ is injective, implying (i).

(ii) Without loss of generality, one may assume that $\Gamma$ acts effectively on $\Sigma$. Then the statement follows by showing that $\Gamma$ acts freely on $\Sigma$. Assume by contradiction that this is not the case. Then there exists $\gamma \in \Gamma$ whose fixed point set $\operatorname{Fix}(\gamma):=\{\zeta \in \Sigma: \gamma \cdot \zeta=\zeta\}$ is not empty. Since $\operatorname{Fix}(\gamma)$ is a proper analytic subset of $\Sigma$, it has no interior point. In particular there exist a $\zeta \in \operatorname{Fix}(\gamma)$ and a sequence $\left\{\zeta_{n}\right\}_{n}$ in the complement of $\operatorname{Fix}(\gamma)$ in $\Sigma$ such that $\zeta_{n} \rightarrow \zeta$. Note that by the continuity of $\gamma$, one has $\gamma \cdot \zeta_{n} \rightarrow \gamma \cdot \zeta=\zeta$.

Let $U$ be an open neighborhood of $\zeta$ on which $\pi$ is injective. Then, for $n$ large enough, both $\zeta_{n}$ and $\gamma \cdot \zeta_{n}$ lie in $U$. Since $\Gamma$ acts trivially on $\Sigma / \Gamma$, it follows that $\pi\left(\zeta_{n}\right)=\gamma \cdot \pi\left(\zeta_{n}\right)=\pi\left(\gamma \cdot \zeta_{n}\right)$. On the other hand since $\zeta_{n} \notin \operatorname{Fix}(\gamma)$, one has $\gamma \cdot \zeta_{n} \neq \zeta_{n}$. This gives the desired contradiction.

Recall the following consequence of the uniqueness of path-liftings on Riemann domains, which will be often used in the proof of the main theorem of this section.

Lemma 7.5. Let $q: \Sigma \rightarrow Z$ be a Riemann domain, and let $W$ be a domain of $\Sigma$ such that the restriction $q \mid W: W \rightarrow Z$ is bijective. Then $W=\Sigma$.

Next comes the main result of this section. 
Theorem 7.6. Let $G / K$ be a noncompact, rank-one, Riemannian symmetric space. Assume that $G$ is a connected, simple, real Lie group that is embedded in its universal complexification $G^{\mathbb{C}}$ and is different from $\operatorname{SL}(2, \mathbb{R})$. Then a holomorphically separable, G-equivariant Riemann domain $q: \Sigma \rightarrow G^{\mathbb{C}} / K^{\mathbb{C}}$ is univalent.

Proof. Recall that $\Sigma$ admits a $G$-equivariant holomorphic embedding into its envelope of holomorphy. Thus we may assume that $\Sigma$ is Stein; see Section 2. We prove the theorem in the case when the $G$-orbit diagram of $G^{\mathbb{C}} / K^{\mathbb{C}}$ is of type (4-9), namely, for $\mathfrak{g}=\mathfrak{s u}(n, 1)$. In all remaining cases but $G=\operatorname{Spin}(3,1)$, which is discussed separately, the statement follows from the same arguments with fewer steps.

So we first assume that $G$ is different from $\operatorname{Spin}(3,1)$ and divide the proof in three subcases, depending on the image of $\Sigma$ in $G^{\mathbb{C}} / K^{\mathbb{C}}$. Finally we discuss the case $G=\operatorname{Spin}(3,1)$.

Case (i): The image $q(\Sigma)$ contains the singular orbit $G \cdot z_{2}$. We begin by proving that there exists a $G$-invariant domain $V \subset \Sigma$ with the properties that $q$ is injective on $V$ and

$$
q(V)=G \cdot\left(\ell_{1}(1) \bigcup_{j=1}^{4}\left(\ell_{j}\left(I_{j}\right) \cup w_{j}\right) \cup z_{2}\right) .
$$

The extended slices $\ell_{j}: \hat{I}_{j} \rightarrow G^{\mathbb{C}} / K^{\mathbb{C}}$ are defined in (7-1). Let $\zeta_{2}$ be an element in $q^{-1}\left(z_{2}\right)$, and let $U$ be an open neighborhood of $\zeta_{2}$ in $\Sigma$ on which the restriction $q \mid U$ is injective. Since the map $q$ is open, the image $q(U)$ intersects the slices for principal orbits starting at $z_{2}$ in the sets $\ell_{j}((0, \epsilon))$ for $j=1, \ldots, 4$ and some $\epsilon>0$. The image $q(U)$ also intersects all nonclosed $G$-orbits containing $G \cdot z_{2}$ in their closures. By Lemma 7.1, each extended slice $\ell_{j}$ admits a lifting $\tilde{\ell}_{j}: \hat{I}_{j} \rightarrow \Sigma$ such that $\tilde{\ell}_{j}(t)=(q \mid U)^{-1} \ell_{j}(t)$ for $t \in(0, \epsilon)$. For $j=1, \ldots, 4$, choose points $\eta_{j} \in(q \mid U)^{-1}\left(G \cdot w_{j}\right)$. Consider then the open $G$-invariant set in $\Sigma$ given by

$$
V:=G \cdot\left(\tilde{\ell}_{1}(1) \bigcup_{j=1}^{4}\left(\tilde{\ell}_{j}\left(I_{j}\right) \cup \eta_{j}\right) \cup \zeta_{2}\right) .
$$

Since $q$ is injective on each lifted slice $\tilde{\ell}_{j}$ and on all $G$-orbits (see Proposition 5.7), it is injective on $V$ as well. Hence $V$ is the open $G$-invariant domain in $\Sigma$ with the required properties.

Consider a sequence $\left\{z_{n}\right\}$ in $G \cdot \ell_{3}(J)$ that converges to a point on the boundary orbit $G \cdot w_{5}$. Recall that the Levi form of $G \cdot w_{5}$ is indefinite; see Remark 9.10. Then, by arguing as in the proof of Lemma 7.1(ii), the domain $V$ can be enlarged to an invariant domain $W$ in $\Sigma$ with the properties that the restriction $q \mid W$ is injective and $q(W)=G^{\mathbb{C}} / K^{\mathbb{C}}$. By Lemma 7.5, one has $W=\Sigma$, and the theorem follows. 
Case (ii): The image $q(\Sigma)$ does not contain the orbit $G \cdot z_{2}$, but contains a nonclosed G-orbit. Assume for example that $w_{1} \in q(\Sigma)$, and let $\eta_{1} \in q^{-1}\left(w_{1}\right)$. By the $G$-equivariance of $q$, by the description of the slice representation at $z_{2}$ given in Remark 4.2, and by Proposition 5.7, there exists a $G$-invariant neighborhood $V$ of $\eta_{1}$ in $\Sigma$ on which $q$ is injective. Its image $q(V)$ intersects the slices $\ell_{1}$ and $\ell_{2}$ in the sets $\ell_{1}((0, \epsilon))$ and $\ell_{2}((0, \epsilon))$ for some $\epsilon>0$. Arguing as in the previous case, one can construct a $G$-invariant domain $V \subset \Sigma$ with the properties that $q$ is injective on $V$ and $q(V)=G \cdot\left(\ell_{1}\left(\hat{I}_{1}\right) \cup w_{1} \cup \ell_{2}\left(\hat{I}_{2}\right)\right)$.

If $V=\Sigma$ (this is possible by Theorem 6.1), then the map $q$ is injective, as desired. If $V \neq \Sigma$, then there exists a point $\eta$ in the closure of $V$ in $\Sigma$ that is mapped by $q$ into one of the nonclosed orbits $G \cdot w_{2}$ or $G \cdot w_{4}$. Assume that $q(\eta)$ lies in $G \cdot w_{4}$. Then by Lemma 7.3(i), the image $q(\Sigma)$ necessarily contains $G \cdot z_{2}$, contradicting the current assumption.

If $q(\eta) \in G \cdot w_{2}$, then by iterating the procedure of lifting slices and orbits we can enlarge $V$ to an invariant domain $W$ in $\Sigma$ on which $q$ is injective and such that

$$
q(W)=G \cdot\left(\ell_{1}\left(\hat{I}_{1}\right) \cup w_{1} \cup \ell_{2}\left(\hat{I}_{2}\right) \cup w_{2} \cup \ell_{3}\left(\hat{I}_{3}\right)\right) .
$$

In particular, $W$ is biholomorphic to $q(W)$, which is not Stein by Theorem 6.1. Hence $W$ is a proper subset of $\Sigma$, and there exists a point $\eta$ in the closure of $W$ in $\Sigma$ whose image $q(\eta)$ lies either in $G \cdot w_{3}$ or in $G \cdot w_{4}$. In both cases, Lemma 7.3 implies that $q(\Sigma)$ contains $G \cdot z_{2}$, contradicting the current assumption. In conclusion, if $q(\Sigma)$ does not contain the singular orbit $G \cdot z_{2}$ but contains the nonclosed orbit $G \cdot w_{1}$, then $q$ is injective. For the other nonclosed $G$-orbits, the theorem can be proved by arguing in a similar way.

Case (iii): The image $q(\Sigma)$ contains no nonclosed $G$-orbits. This assumption implies that the image $q(\Sigma)$ contains none of the singular orbits lying in the closure of a nonclosed $G$-orbit. More precisely, $q(\Sigma)$ contains neither $G \cdot z_{2}$ nor $G \cdot z_{3}$. Note that the hypersurfaces $G \cdot\left(z_{2} \bigcup_{j=1}^{4} w_{j}\right)$ and $G \cdot\left(z_{3} \cup w_{5}\right)$ disconnect $G^{\mathbb{C}} / K^{\mathbb{C}}$. Therefore there exists a slice $\ell=\ell_{j}$ for some $j=1, \ldots, 5$ such that $q(\Sigma)=G \cdot \ell(J)$ for some interval $J \subset \hat{I}$ that is open in $\hat{I}$. Define $M:=q^{-1}(\ell(J))$. One has that $\Sigma=G \cdot M$. Moreover, since $q$ is injective on $G$-orbits (see Proposition 5.7) and every orbit in $q(\Sigma)$ intersects $\ell(J)$ in a single point, every $G$-orbit in $\Sigma$ intersects $M$ in a single point as well. As a consequence, the surjective map $\Pi: \Sigma \mapsto M$ given by $\zeta \mapsto G \cdot \zeta \cap M$ is well defined.

Claim. The map $\Pi$ is continuous.

Proof of the claim. Let $N$ be an open set in $M$. We prove the claim by showing that for every $m \in N$ and $\zeta \in \Pi^{-1}(m)$, there exists an open neighborhood of $\zeta$ in $\Sigma$ which is contained in $\Pi^{-1}(N)$. By construction, $\zeta=g \cdot m$ for some $g \in G$. Let $V$ be an open neighborhood of $m$ in $\Sigma$ on which $q$ is injective. Choose an open 
interval $J^{\prime} \subset J$ such that $q(m) \in \ell\left(J^{\prime}\right) \subset q(V)$. Note that $q(m)$ either sits on a principal $G$-orbit or on the singular orbit $G \cdot z_{1} \cong G / K$. Let $\tilde{\ell}\left(J^{\prime}\right)$ be the lifting of $\ell\left(J^{\prime}\right)$ via the restriction $q \mid V$. By shrinking $J^{\prime}$ if necessary, one can find an open neighborhood $U$ of the identity in $G$ such that $U \cdot \ell\left(J^{\prime}\right)$ is open and contained in $q(V)$. This fact is clear if $q(m)$ lies on a principal $G$-orbit; see diagram (4-9). If $q(m)$ lies on the singular orbit $G \cdot z_{1}$, it follows from the equivariant embedding (4-1) at $z_{1}$ and the compactness of the isotropy subgroup $G_{z_{1}} \cong K$.

As a result, $U \cdot \tilde{\ell}\left(J^{\prime}\right)=(q \mid V)^{-1}\left(U \cdot \ell\left(J^{\prime}\right)\right)$ is an open neighborhood of $m$ in $\Sigma$, and $g U \cdot \tilde{\ell}\left(J^{\prime}\right)$ is an open neighborhood of $\zeta$ contained in $\Pi^{-1}(N)$. Hence $\Pi^{-1}(N)$ is open in $\Sigma$, as wished (one can show that $M \cong \Sigma / G$ and that $\Pi$ can be identified with the quotient map).

By the above claim, $M$ is connected and is a one-dimensional real-analytic submanifold of $\Sigma$. It follows that $q$ is injective on $M$. Moreover $M$ and $q(M)$ are slices for the $G$-action in $\Sigma$ and $q(\Sigma)$, respectively. Since $q$ is injective on $G$-orbits, it is injective on $\Sigma$ implying the theorem.

Case (iv): The group $G$ is $\operatorname{Spin}(3,1)$. Assume by contradiction that $q: \Sigma \rightarrow$ $G^{\mathbb{C}} / K^{\mathbb{C}}$ is not univalent. Recall that the center of $G$ acts trivially on $G^{\mathbb{C}} / K^{\mathbb{C}}$ and that by Cases (i)-(iii), the statement holds true for the group $\operatorname{SO}_{0}(3,1)$. Then Lemma 7.4 applies to show that the restriction of $q$ to every $G$-orbit is a double covering and the image $q(\Sigma)$ is Stein. On the other hand, by Theorem 6.1, all Stein $G$-invariant domains in $G^{\mathbb{C}} / K^{\mathbb{C}}$ contain a singular orbit diffeomorphic to $G / K$. Since $G / K$ is simply connected, this gives a contradiction. This proves the theorem.

When $G=\operatorname{SL}(2, \mathbb{R})$, noninjective, Stein $G$-equivariant Riemann domains over $G^{\mathbb{C}} / K^{\mathbb{C}}$ do exist. Next we construct one such Riemann domain explicitly. It turns out that such an example is essentially the only possible one. Indeed by Lemma 7.4, if $q: \Sigma \rightarrow G^{\mathbb{C}} / K^{\mathbb{C}}$ is a Stein, $G$-equivariant Riemann domain that is not univalent, then the center $\Gamma=\left\{ \pm I_{2}\right\}$ acts freely on $\Sigma$. Moreover, $q$ is a $G$-equivariant covering onto its image $q(\Sigma)$ that turns out to be Stein. It follows that the restriction of $q$ to every $G$-orbit is a double covering. Thus the singular orbits $G \cdot z_{1}$ and $G \cdot z_{3}$, which are simply connected, cannot lie in $q(\Sigma)$. Then, by Theorem 6.1, the image $q(\Sigma)$ coincides with a domain $S_{i}(b)$ for some $i=1,2$ and $b \geq 0$. For every $S_{i}(b)$ there is exactly one $G$-equivariant double covering. In the example below, we carry out its construction for $q(\Sigma)=S_{1}(0)$.

Example 7.7. Let $G=\operatorname{SL}(2, \mathbb{R})$. Consider the Stein domain $S_{1}(0)$ in $G^{\mathbb{C}} / K^{\mathbb{C}}$ defined in (6-1). Let

$$
\ell_{2}: \mathbb{R}^{>0} \rightarrow G^{\mathbb{C}} / K^{\mathbb{C}} \quad \text { and } \quad \ell_{2}(s):=\exp (\text { is } C) z_{2}
$$


be the slice map defined in (4-6). The isotropy subgroup in $G$ of every point $\ell_{2}(s)$ coincides with $\left\{ \pm I_{2}\right\}$; see Remarks 5.4 and 4.1. It follows that $S_{1}(0):=G \cdot \ell_{2}\left(\mathbb{R}^{>0}\right)$ is topologically equivalent to $\operatorname{SO}_{0}(2,1) \times \mathbb{R}^{>0}$. Define $\Sigma:=G \times \mathbb{R}^{>0}$. Since $G$ is a double covering of $\mathrm{SO}_{0}(2,1)$, the map

$$
q: \Sigma \rightarrow S_{1}(0), \quad(g, s) \mapsto g \ell_{2}(s)
$$

defines a double covering of $S_{1}(0)$. As a consequence, with the complex structure pulled back from $S_{1}(0)$, the manifold $\Sigma$ is Stein; see [Stein 1956]. Also the map $q$ is a holomorphic covering. In other words, $q: \Sigma \rightarrow S_{1}(0)$ defines a nonunivalent Stein, $G$-equivariant Riemann domain over $G^{\mathbb{C}} / K^{\mathbb{C}}$.

Remark 7.8. By the results of Lemma 7.4, one can show that Theorem 7.6 also holds for $G$ not embedded in $G^{\mathbb{C}}$, provided that the center $\Gamma$ of $G$ is finite and $G$ is not a covering of $\operatorname{SL}(2, \mathbb{R})$ (see Case (iv) in the proof of Theorem 7.6). If $G$ is a covering of $\operatorname{SL}(2, \mathbb{R})$, a construction similar to the one in Example 7.7 yields a nonunivalent, Stein $G$-equivariant Riemann domain over $G^{\mathbb{C}} / K^{\mathbb{C}}$.

As an application of Theorem 7.6 and the classification of all Stein $G$-invariant domains in $G^{\mathbb{C}} / K^{\mathbb{C}}$ given in Section 6, we now exhibit a family of Kobayashi hyperbolic $G$-invariant subdomains of $\mathrm{SU}(1,1)^{\mathbb{C}} / \mathrm{U}(1)^{\mathbb{C}}$ whose envelopes of holomorphy are not Kobayashi hyperbolic.

Example 7.9. Let $G=\mathrm{SU}(1,1)$, and let $W_{1,1}:=D_{1}(0) \cup G \cdot w_{1} \cup S_{1}(0)$ be the Stein $G$-invariant domain defined in Example 6.3. Recall that $W_{1,1}$ is biholomorphic to $\Delta \times \mathbb{C}$ via the map

$$
F: \Delta \times \mathbb{C} \rightarrow W_{1,1}, \quad(u, v) \mapsto([u: 1],[\bar{v}: 1+\bar{u} \bar{v}]) .
$$

Consider its invariant subdomains given by

$$
D_{c}:=D_{1}(0) \cup G \cdot w_{1} \cup G \cdot \ell_{2}(0, c) \text { for } 0<c<\infty .
$$

Denote by $\tilde{f}$ the pull-back via $F$ of the $G$-invariant function $f$ defined in (6-2). Then

$$
\tilde{f}(u, v)=-\left(1-|u|^{2}\right)\left(|1+u v|^{2}-|v|^{2}\right),
$$

and $D_{\mathfrak{c}}$ is biholomorphic to a sublevel set $B_{R}=\{\tilde{f}<R\}$ in $\Delta \times \mathbb{C}$ for some $R>0$. Consider the holomorphic projection $\pi: B_{R} \rightarrow \Delta$ onto the first factor. An easy computation shows that, for $u \in \Delta$, the preimage $\pi^{-1}(u)$ is a disk in $\mathbb{C}$ of center $(\operatorname{Re} u,-\operatorname{Im} u) /\left(1-|u|^{2}\right)$ and radius $(1+R) /\left(1-|u|^{2}\right)^{2}$. It follows that for every $u \in \Delta$ there exists a neighborhood $U$ of $u$ such that $\pi^{-1}(U)$ is Kobayashi hyperbolic. Then, by [Kobayashi 1998, Theorem 3.2.14], the domains $B_{r}$ and $D_{c}$ are Kobayashi hyperbolic as well.

Finally from Theorem 7.6 and Theorem 6.1, it follows that the envelope of holomorphy of $D_{c}$ is given by $W_{1,1}$. In particular, it is not Kobayashi hyperbolic. 


\section{Univalence over $G^{\mathbb{C}}$}

Let $G$ be a connected, noncompact, real simple Lie group, let $K \subset G$ be a maximal compact subgroup, and let $G^{\mathbb{C}}$ be its universal complexification. In this section we prove a univalence result for $G \times K$-equivariant Riemann domains over $G^{\mathbb{C}}$ when the symmetric space $G / K$ has rank one. We also discuss some examples.

Theorem 8.1. Let $G / K$ be a noncompact, rank-one, Riemannian symmetric space. Assume that $G$ is a connected, simple, real Lie group that has finite center and is not a covering of $\mathrm{SL}(2, \mathbb{R})$. Then a holomorphically separable, $G \times K$-equivariant Riemann domain $p: Y \rightarrow G^{\mathbb{C}}$ is univalent.

Proof. Recall that $Y$ admits a $G \times K$-equivariant holomorphic embedding into its envelope of holomorphy. Thus we may assume that $Y$ is Stein (see Section 2). Consider the induced Stein, $G$-equivariant Riemann domain $q: Y / / K \rightarrow G^{\mathbb{C}} / K^{\mathbb{C}}$ constructed in Section 3. By Theorem 7.6 and Remark 7.8 the map $q$ is injective. Then, by Corollary 3.3 the Riemann domain $p: Y \rightarrow G^{\mathbb{C}}$ is univalent, as wished.

When $G$ is either $\operatorname{SL}(2, \mathbb{R})$ or a nontrivial covering of $\operatorname{SL}(2, \mathbb{R})$, a construction similar to the one in Example 7.7 yields examples of nonunivalent, Stein, $G \times K$ equivariant Riemann domains over $G^{\mathbb{C}}$.

Example 8.2. Let $G=\operatorname{SL}(2, \mathbb{R})$, and let $S_{1}(0)$ be the Stein, $G$-invariant domain in $G^{\mathbb{C}} / K^{\mathbb{C}}$ defined in (6-1). As we observed in Example 7.7, the domain $S_{1}(0)$ is diffeomorphic to $\operatorname{SO}_{0}(2,1) \times \mathbb{R}^{>0}$. Define $\Omega:=\pi^{-1}\left(S_{1}(0)\right)$, where $\pi: G^{\mathbb{C}} \rightarrow$ $G^{\mathbb{C}} / K^{\mathbb{C}}$ is the canonical projection. Since $\pi$ is holomorphic and both $S_{1}(0)$ and $G^{\mathbb{C}}$ are Stein, the domain $\Omega$ is Stein as well. Consider the slice $\ell_{2}: \mathbb{R}^{>0} \rightarrow G^{\mathbb{C}} / K^{\mathbb{C}}$ (see (4-6)) and its lifting to $G^{\mathbb{C}}$ defined by $\tilde{\ell}_{2}(s):=\exp (i s C) \exp \left(i A_{2}\right)$. Define $Y:=G \times \mathbb{R}^{>0} \times K^{\mathbb{C}}$. Note that the map

$$
p: Y \rightarrow \Omega, \quad(g, s, k) \mapsto g \tilde{\ell}_{2}(s) k^{-1}
$$

is a double covering. With the complex structure pulled back from $\Omega$, the manifold $Y$ is Stein; see [Stein 1956]. Also, the map $p$ is holomorphic. Let $G \times K$ act on $Y$ by $(l, h) \cdot(g, s, k):=(l g, s, h k)$ and on $\Omega$ by left and right translations. Then $p$ defines a nonunivalent, Stein, $G \times K$-equivariant Riemann domain over $G^{\mathbb{C}}$.

Let $G=K \times N$ be the product of a compact Lie group and a simply connected nilpotent Lie group. Then a holomorphically separable, $G$-equivariant Riemann domain over $G^{\mathbb{C}}$ is necessarily univalent; see [Cœuré and Loeb 1986; Iannuzzi 1999; Casadio Tarabusi et al. 2000]. The above example shows that an analogous statement does not hold for a semisimple Lie group $G$. Next we exhibit a different counterexample for $G=\mathrm{SO}_{0}(2,1)$, a group that meets the assumptions of Theorem 8.1. Such an example was pointed out to us by K. Oeljeklaus. We are not 
aware of similar constructions in higher dimension. That is, if the dimension of $G / K$ is greater than two, univalence of holomorphically separable, $G$-equivariant Riemann domains over $G^{\mathbb{C}}$ seems to be an open question.

Example 8.3. Let $G=\mathrm{SO}_{0}(2,1)$. Then $G^{\mathbb{C}}=\operatorname{SO}(2,1, \mathbb{C})$ and $K^{\mathbb{C}}=\mathrm{SO}(2, \mathbb{C})$. Let $S_{1}(0)$ be the $G$-invariant Stein domain in $G^{\mathbb{C}} / K^{\mathbb{C}}$ defined in (6-1), and let $\Omega=\pi^{-1}\left(S_{1}(0)\right)$, where $\pi: G^{\mathbb{C}} \rightarrow G^{\mathbb{C}} / K^{\mathbb{C}}$ is the canonical projection. As we already observed in Example 8.2, the domain $\Omega$ is a Stein, $G$-invariant domain in $G^{\mathbb{C}}$ which is diffeomorphic to $G \times \mathbb{R}^{>0} \times K^{\mathbb{C}}$. Denote by $\widetilde{K}^{\mathbb{C}}$ the universal covering of $K^{\mathbb{C}}$ and by $\psi: \widetilde{K}^{\mathbb{C}} \rightarrow K^{\mathbb{C}}$ the covering homomorphism. Let $Y:=G \times \mathbb{R}^{>0} \times \widetilde{K}^{\mathbb{C}}$, and let $G$ act on $Y$ by left translations. Consider the slice $\ell_{2}: \mathbb{R}^{>0} \rightarrow G^{\mathbb{C}} / K^{\mathbb{C}}$ (see (4-6)) and its lifting to $G^{\mathbb{C}}$ given by $\tilde{\ell}_{2}(s):=\exp (i s C) \exp \left(i A_{2}\right)$. Define a $G$ equivariant covering of $\Omega$ by

$$
p: Y \rightarrow \Omega, \quad(g, s, k) \mapsto g \tilde{\ell}_{2}(s) \psi\left(k^{-1}\right) .
$$

With the complex structure pulled back from $\Omega$, the manifold $Y$ is Stein; see [Stein 1956]. Also the map $p$ is holomorphic. In particular $p: Y \rightarrow \Omega$ defines a nonunivalent, Stein, $G$-equivariant Riemann domain over $G^{\mathbb{C}}$.

Remark. One can show that $\Omega$ is a holomorphically trivial $\mathbb{C}^{*}$-bundle over $S_{1}(0)$. Thus it is biholomorphic to $S_{1}(0) \times \mathbb{C}^{*}$, and consequently $Y$ is biholomorphic to $S_{1}(0) \times \mathbb{C}$. After identifying $S_{1}(0)$ with $\operatorname{SO}_{o}(2,1) \times \mathbb{R}^{>0}$, one sees that the map $\mathrm{SO}_{o}(2,1) \times \mathbb{R}^{>0} \rightarrow G^{\mathbb{C}}$ given by $(g, s) \mapsto g \tilde{\ell}_{2}(s)$ defines a global $C^{\infty}$-section of the holomorphic $\mathbb{C}^{*}$-bundle $\left.\pi\right|_{\Omega}: \Omega \rightarrow S_{1}(0)$. Hence such bundle is differentiably trivial and, by the Oka principle, is also holomorphically trivial [Grauert 1958], as claimed. For completeness, we explicitly construct a trivialization on the model of $G^{\mathbb{C}} / K^{\mathbb{C}}$ discussed in Example 4.7 and Remark 4.8.

Let $G=\mathrm{SU}(1,1)$ and identify $G^{\mathbb{C}} / K^{\mathbb{C}}$ with $\mathbb{P}^{1} \times \overline{\mathbb{P}}^{1} \backslash\left\{\langle z, w\rangle_{1,1}=0\right\}$. Note that $S_{1}(0)$ corresponds to the subset $\{([1: u],[\bar{v}: 1]): u, v \in \Delta, u \neq v\}$; see Example 6.3. Let $D$ be the diagonal in $\Delta \times \Delta$. Then the injective holomorphic map

$$
\Delta \times \Delta \backslash D \rightarrow \mathbb{P}^{1} \times \overline{\mathbb{P}}^{1} \backslash\left\{\langle z, w\rangle_{1,1}=0\right\}, \quad(u, v) \mapsto([1: u],[\bar{v}: 1])
$$

identifies $\Delta \times \Delta \backslash D$ with $S_{1}(0)$. The map

$$
\Delta \times \Delta \backslash D \rightarrow G^{\mathbb{C}}, \quad(u, v) \mapsto\left(\begin{array}{cc}
1 & 1 /(u-v) \\
v & u /(u-v)
\end{array}\right)=: M(u, v)
$$

defines a global holomorphic section of the $\mathbb{C}^{*}$-bundle $\left.\pi\right|_{\Omega}: \Omega \rightarrow S_{1}(0)$, since one has $M(u, v) \cdot([0: 1],[0: 1])=([1: u],[\bar{v}: 1])$. As a consequence the map

$$
(\Delta \times \Delta) \backslash D \times \mathbb{C}^{*} \rightarrow \Omega, \quad(u, v, \lambda) \rightarrow M(u, v) \operatorname{diag}\left(\lambda^{-1}, \lambda\right)
$$


defines a biholomorphism from $S_{1}(0) \times \mathbb{C}^{*}$ onto $\Omega$.

\section{Appendix: The Levi form of nonclosed hypersurface orbits}

In this section we outline the computation of the Levi form of nonclosed hypersurface $G$-orbits in $G^{\mathbb{C}} / K^{\mathbb{C}}$. We used the results in Section 6 to complete the classification of Stein $G$-invariant domains in $G^{\mathbb{C}} / K^{\mathbb{C}}$. Recall that every real hypersurface $S$ in a complex manifold inherits a CR-structure of hypersurface type. Let $J$ denote the complex structure of the ambient manifold. For every $x \in S$, the tangent space to $S$ at $x$ decomposes as $T S_{x}=T_{\mathbb{C}} S_{x} \oplus N S_{x}$, where $T_{\mathbb{C}} S_{x}=T S_{x} \cap J\left(T S_{x}\right)$ is a complex subspace of $T S_{x}$, called the complex tangent space, and $N S_{x}$ is a one-dimensional real subspace. Denote by $T S=T_{\mathbb{C}} S \oplus N S$ the tangent bundle of $S$. The subbundle $\left(T_{\mathbb{C}} S\right)^{\mathbb{C}} \subset T S^{\mathbb{C}}$ of the complexified tangent bundle $T S^{\mathbb{C}}$ decomposes as $H S \oplus A S$, where $H S$ and $A S$ denote its $(1,0)$ and $(0,1)$ components, respectively. Let $Z$ be a tangent vector in $T_{\mathbb{C}} S_{x}$ and $\widehat{Z}$ an arbitrary extension of $Z$ to a local section of $T_{\mathbb{C}} S$. Then the vector fields

$$
\frac{1}{2}(\widehat{Z}-i J \widehat{Z}) \text { and } \frac{1}{2}(\widehat{Z}+i J \widehat{Z})
$$

define local sections of the bundles $H S$ and $A S$, respectively. The Levi form of $S$ at $z$ is the hermitian form $L_{x}: T_{\mathbb{C}} S_{x} \times T_{\mathbb{C}} S_{x} \rightarrow\left(T S_{x}\right)^{\mathbb{C}} /\left(T_{\mathbb{C}} S_{x}\right)^{\mathbb{C}}$ defined by

$$
L_{x}(Z, W,):=\frac{i}{4}[\widehat{Z}-i J \widehat{Z}, \widehat{W}+i J \widehat{W}]_{x} \bmod \left(T_{\mathbb{C}} S\right)^{\mathbb{C}} .
$$

In the hypersurface case, $\left(T S_{x}\right)^{\mathbb{C}} /\left(T_{\mathbb{C}} S_{x}\right)^{\mathbb{C}}$ is a one-dimensional complex vector space. When $Z$ varies in $T_{\mathbb{C}} S_{x}$, the image of the quadratic form $L_{x}(Z, Z$, ) is contained in its real part, which can be identified with $N S_{x} \cong \mathbb{R}$. We say that the Levi form of $S$ is definite if $\left\{L_{x}(Z, Z)\right\}$ is a halfline in $N S_{x}$, that it is indefinite if $\left\{L_{x}(Z, Z)\right\}$ coincides with $N S_{x}$, and that it is identically zero if $\left\{L_{x}(Z, Z)\right\}=\{0\}$; for more details, see [Boggess 1991].

9.1. Nonclosed orbits with a totally real singular orbit in their closure. We first consider nonclosed $G$-orbits that contain in their closure the orbit of a point $z=$ $\exp i A K^{\mathbb{C}} \in A_{0}$, satisfying the condition $\alpha(A)=\pi / 2$, with $\alpha$ a simple restricted root; see (4-2) and (4-7). The singular orbit $G \cdot z$ is diffeomorphic to a rankone, pseudo-Riemannian symmetric space $G / H$, embedded in $G^{\mathbb{C}} / K^{\mathbb{C}}$ as a totally real submanifold of maximal dimension. Let $\left(\mathfrak{g}=\mathfrak{h} \oplus \mathfrak{q}, \tau_{z}\right)$ be the corresponding symmetric algebra. Nonclosed $G$-orbits in $G^{\mathbb{C}} / K^{\mathbb{C}}$ containing $G \cdot z$ in their closure are in one-to-one correspondence with the nilpotent $\operatorname{Ad}_{H}$-orbits in $\mathfrak{q}$; see (4-1) and Remark 4.2.

Let $X$ be an element in $\mathfrak{q}$, and let $x=\exp i X \cdot z$ be the corresponding point in $G^{\mathbb{C}} / K^{\mathbb{C}}$. Denote by $S$ the $G$-orbit of $x$. Denote by $\pi: G^{\mathbb{C}} \rightarrow G^{\mathbb{C}} / K^{\mathbb{C}}$ the 
canonical projection and by $\pi_{*}$ its differential. Then the tangent space to $S$ at $x$ is generated by the vector fields induced on $G^{\mathbb{C}} / K^{\mathbb{C}}$ by the one-parameter subgroups in $G$, via the map

$$
*: \mathfrak{g} \rightarrow T\left(G^{\mathbb{C}} / K^{\mathbb{C}}\right)_{x}, \quad X \mapsto X^{*}:=\left(\pi_{*}\right)_{x}\left(\left.\frac{d}{d t}\right|_{t=0} \exp t X\right) .
$$

Observe that $T\left(G^{\mathbb{C}} / K^{\mathbb{C}}\right)_{z} \cong \mathfrak{q}^{\mathbb{C}}$ and $T\left(G^{\mathbb{C}} / K^{\mathbb{C}}\right)_{x} \cong \operatorname{Ad}_{x} \mathfrak{q}^{\mathbb{C}}$. Hence the vector $X^{*}$ is the $\operatorname{Ad}_{x} \mathfrak{q}^{\mathbb{C}}$-component of $X$ in the decomposition $\mathfrak{g}^{\mathbb{C}}=\operatorname{Ad}_{x} \mathfrak{h}^{\mathbb{C}} \oplus \operatorname{Ad}_{x} \mathfrak{q}^{\mathbb{C}}$.

To explicitly determine base points for such nonclosed orbits and their tangent spaces, we decompose $\mathfrak{g}$ by an appropriate restricted root system. Fix a maximal abelian subalgebra $\mathfrak{b} \subset \mathfrak{h} \cap \mathfrak{p}$. Because $\mathfrak{g}$ is of real rank one, $\operatorname{dim} \mathfrak{b}=1$ and $Z_{\mathfrak{g}}(\mathfrak{b})=\mathfrak{b} \oplus Z_{\mathfrak{k}}(\mathfrak{b})$. Let $\Delta_{\mathfrak{b}}$ be the restricted root system of $\mathfrak{g}$ with respect to $\mathfrak{b}$, and let $\mathfrak{g}=\mathfrak{g}^{0} \oplus \mathfrak{g}^{ \pm \lambda} \oplus \mathfrak{g}^{ \pm 2 \lambda}$ and $\mathfrak{g}^{0}=Z_{\mathfrak{g}}(\mathfrak{b})$ be the corresponding restricted root decomposition. Every root space $\mathfrak{g}^{\lambda}$ is $\tau_{z}$-stable. For every $\mu \in \Delta_{\mathfrak{b}} \cup\{0\}$, we indicate by $\mathfrak{g}_{\mathfrak{h}}^{\mu}$ and $\mathfrak{g}_{\mathfrak{q}}^{\mu}$ the intersections of $\mathfrak{g}^{\mu}$ with $\mathfrak{h}$ and $\mathfrak{q}$, respectively. In particular, we have a combined decomposition

(9-2) $\mathfrak{g}=\mathfrak{h} \oplus \mathfrak{q}, \quad$ where $\mathfrak{h}=\mathfrak{g}_{\mathfrak{h} \cap \mathfrak{k}}^{0} \oplus \mathfrak{g}_{\mathfrak{h}}^{ \pm \lambda} \oplus \mathfrak{g}_{\mathfrak{h}}^{ \pm 2 \lambda} \oplus \mathfrak{b}$ and $\mathfrak{q}=\mathfrak{g}_{\mathfrak{q} \cap \mathfrak{k}}^{0} \oplus \mathfrak{g}_{\mathfrak{q}}^{ \pm \lambda} \oplus \mathfrak{g}_{\mathfrak{q}}^{ \pm 2 \lambda}$

Here $\mathfrak{g}_{\mathfrak{h} \cap \mathfrak{k}}^{0}$ and $\mathfrak{g}_{\mathfrak{q} \cap \mathfrak{k}}^{0}$ denote the intersections of $Z_{\mathfrak{k}}(\mathfrak{b})$ with $\mathfrak{h}$ and $\mathfrak{q}$, respectively. Note that, by the real rank one condition, $\mathfrak{g}_{\mathfrak{q} \cap \mathfrak{k}}^{0}$ coincides with $\mathfrak{g}_{\mathfrak{q}}^{0}$. If the restricted roots system $\Delta_{\mathfrak{b}}$ is reduced, then $\mathfrak{g}^{ \pm 2 \lambda}=\{0\}$.

Lemma 9.1. Let $\mathfrak{g}$ be a simple real Lie algebra of real rank one with reduced restricted root system (that is, $\mathfrak{g}=\mathfrak{s o}(n, 1)$ ). Then the following facts hold:

(i) $\operatorname{dim} \mathfrak{g}_{\mathfrak{q}}^{ \pm \lambda}=1$.

(ii) $\left[\mathfrak{g}_{\mathfrak{q}}^{\lambda}, \mathfrak{g}_{\mathfrak{h}}^{-\lambda}\right]=\mathfrak{g}_{\mathfrak{q}}^{0} \quad$ and $\quad\left[\mathfrak{g}_{\mathfrak{q}}^{-\lambda}, \mathfrak{g}_{\mathfrak{h}}^{\lambda}\right]=\mathfrak{g}_{\mathfrak{q}}^{0}$

Proof. Observe that $\theta \mathfrak{g}_{\mathfrak{q}}^{\lambda}=\mathfrak{g}_{\mathfrak{q}}^{-\lambda}$. Hence $\mathfrak{g}_{\mathfrak{q}}[\lambda]:=\mathfrak{g}_{\mathfrak{q}}^{\lambda} \oplus \mathfrak{g}_{\mathfrak{q}}^{-\lambda}$ is a $\theta$-stable subspace of $\mathfrak{q}$ and $\operatorname{dim} \mathfrak{g}_{\mathfrak{q}}[\lambda] \cap \mathfrak{p}=\operatorname{dim} \mathfrak{g}_{\mathfrak{q}}^{\lambda}$. Since $\mathfrak{g}_{\mathfrak{q}}^{0} \subset \mathfrak{k}$ and $\operatorname{dim} \mathfrak{p} \cap \mathfrak{q}=1$ (see the proof of Lemma 4.3(ii), statement (i) holds. Statement (ii) can be verified directly.

Lemma 9.2. Let $\mathfrak{g}$ be a real simple Lie algebra of real rank one with nonreduced restricted root system (that is, $\mathfrak{g}=\mathfrak{s u}(n, 1), \mathfrak{s p}(n, 1)$, or $\left.\mathfrak{f}_{4}^{*}\right)$. Then the following facts hold:

(i) The root spaces $\mathfrak{g}^{ \pm 2 \lambda}$ are contained in $\mathfrak{h}$. Therefore $\mathfrak{g}_{\mathfrak{q}}^{ \pm 2 \lambda}=\{0\}$.

(ii) $\operatorname{dim} \mathfrak{g}_{\mathfrak{q}}^{ \pm \lambda}>1$.

(iii) Fix $X_{\lambda}^{0} \in \mathfrak{g}_{\mathfrak{q}}^{\lambda}$ and denote by $\left(\mathfrak{g}_{\mathfrak{q}}^{\lambda}\right)_{0}$ a complement of $\mathbb{R} X_{\lambda}^{0}$ in $\mathfrak{g}_{\mathfrak{q}}^{\lambda}$; denote by $\left(\mathfrak{g}_{\mathfrak{q}}^{-\lambda}\right)_{0}$ a complement of $\mathbb{R} \theta X_{\lambda}^{0}$ in $\mathfrak{g}_{\mathfrak{q}}^{-\lambda}$. Then

$$
\left[X_{\lambda}^{0}, \mathfrak{g}_{\mathfrak{h} \cap \mathfrak{k}}^{0}\right]=\left(\mathfrak{g}_{\mathfrak{q}}^{\lambda}\right)_{0}, \quad\left[X_{\lambda}^{0}, \mathfrak{g}_{\mathfrak{h}}^{-\lambda}\right]=\mathfrak{g}_{\mathfrak{q}}^{0}, \quad\left[X_{\lambda}^{0}, \mathfrak{g}_{\mathfrak{h}}^{-2 \lambda}\right]=\left(\mathfrak{g}_{\mathfrak{q}}^{-\lambda}\right)_{0} .
$$


Proof. Real rank one Lie algebras with a nonreduced restricted root system are equal-rank. Hence the root system $\Delta$ of $\mathfrak{g}^{\mathbb{C}}$, with respect to a maximally split Cartan subalgebra of $\mathfrak{g}$ extending $\mathfrak{b}$, has a real root. Since $\operatorname{dim} \mathfrak{g}^{2 \lambda}$ is odd, the restriction of such a root to $\mathfrak{b}$ coincides with the restricted root $2 \lambda$; see [Helgason 1978, page 584]. Further, by [Geatti 2002, Remark 2.13], the subalgebra $\mathfrak{h}$ is a noncompact real form of $\operatorname{Ad}_{z} \mathfrak{k}^{\mathbb{C}} \cong \mathfrak{k}^{\mathbb{C}}$ with respect to the conjugation $\sigma \tau_{z} \mid \operatorname{Ad}_{z} \mathfrak{k}^{\mathbb{C}}$. Precisely, if $\mathfrak{g}=\mathfrak{s u}(n, 1), \mathfrak{s p}(n, 1)$, or $\mathfrak{f}_{4}^{*}$, then $\mathfrak{h}$ is given by $\mathfrak{u}(n-1,1) \oplus \mathfrak{u}(1)$, $\mathfrak{s p}(n-1,1) \oplus \mathfrak{s p}(1)$, or $\mathfrak{s o}(8,1)$, respectively. Since $\mathfrak{h}$ is equal-rank, the root spaces $\mathfrak{g}^{ \pm 2 \lambda}$ have nontrivial intersection with $\mathfrak{h}$. Statements (i) and (ii) then follow by looking at the dimensions of the restricted root spaces of $\mathfrak{h}$ and $\mathfrak{g}$; (see [Helgason 1978, page 532]). Statement (iii) can be verified directly.

Reference points for nonclosed G-orbits. Let $\mathscr{C}=\exp i \mathfrak{c} \cdot z$ be the standard Cartan subset in $G^{\mathbb{C}} / K^{\mathbb{C}}$ with base point $z$. Recall that $\mathfrak{c}=\mathbb{R}(X+\theta(X))$, where $X$ is a nonzero vector in $\mathfrak{g}^{\alpha}$ (here $\mathfrak{g}^{\alpha}$ is a restricted root space with respect to the adjoint action of $\mathfrak{a} \subset \mathfrak{p}$, as in Section 4). Normalize the triple $\{X, \theta(X), A:=[\theta(X), X]\}$ so that $\alpha(A)=2$. Define $B:=X-\theta(X)$ and $\mathfrak{b}:=\mathbb{R}(X-\theta(X))$. One easily verifies that $\mathfrak{b}$ is a maximal abelian subalgebra in $\mathfrak{h} \cap \mathfrak{p}$. If the restricted root system $\Delta_{\mathfrak{b}}$ is reduced, then

$$
X_{\lambda}^{0}=\frac{1}{2}(A-(X+\theta X)) \quad \text { and } \quad X_{-\lambda}^{0}=\frac{1}{2}(A+(X+\theta X))
$$

are generators of the one-dimensional spaces $\mathfrak{g}_{\mathfrak{q}}^{\lambda}$ and $\mathfrak{g}_{\mathfrak{q}}^{-\lambda}$, respectively. They satisfy the relations

$$
\left[B, X_{\lambda}^{0}\right]=2 X_{\lambda}^{0}, \quad\left[B, X_{-\lambda}^{0}\right]=-2 X_{-\lambda}^{0}, \quad\left[X_{\lambda}^{0}, X_{-\lambda}^{0}\right]=B, \quad \theta X_{\lambda}^{0}=-X_{-\lambda}^{0} .
$$

The vectors $X_{\lambda}^{0}, X_{-\lambda}^{0},-X_{\lambda}^{0}$ and $-X_{-\lambda^{0}}$ are a complete set of representatives of the nilpotent $\operatorname{Ad}_{H}$-orbits in $\mathfrak{q}$. The corresponding points in $G^{\mathbb{C}} / K^{\mathbb{C}}$,

$x_{0}=\exp i X_{\lambda}^{0} \cdot z, \quad x_{1}=\exp i X_{-\lambda}^{0} \cdot z, \quad y_{0}=\exp \left(-i X_{\lambda}^{0}\right) \cdot z, \quad y_{1}=\exp \left(-i X_{-\lambda}^{0}\right) \cdot z$

lie on nonclosed $G$-orbits containing the singular orbit $G \cdot z$ in their closures. In the orbit diagram (4-3), the $G$-orbits of $x_{0}, x_{1}, y_{0}, y_{1}$ are represented by $w_{3}, w_{2}, w_{1}$, $w_{4}$, respectively. If $\operatorname{dim} G / K>2$, the points $x_{0}$ and $x_{1}$ lie on the same $G$-orbit and likewise the points $y_{0}$ and $y_{1}$; see diagram (4-4). When the restricted root system $\Delta_{\mathfrak{b}}$ is nonreduced, all points $x=\exp i X_{\lambda} \cdot z$ with $X_{\lambda} \in \mathfrak{g}_{\mathfrak{q}}^{\lambda}$ and $y=\exp i X_{-\lambda} \cdot z$ with $X_{-\lambda} \in \mathfrak{g}_{\mathfrak{q}}^{-\lambda}$ lie on the same $G$-orbit. They are represented by $w_{5}$ in the orbit diagrams (4-9) and (4-10).

Remark 9.3. When the restricted root system $\Delta_{\mathfrak{b}}$ is reduced, the points $x_{0}$ and $x_{1}$ lie on the boundary of the Stein domain $D_{2}(0)$. The points $y_{0}$ and $y_{1}$ lie on the boundary of the Stein domain $D_{1}(0)$; see (6-1). 
The tangent space to the G-orbit of $x_{0}$. Denote by $S$ the $G$-orbit of the point $x_{0}=\exp i X_{\lambda}^{0} \cdot z$ with $X_{\lambda}^{0} \in \mathfrak{g}_{\mathfrak{q}}^{\lambda}$. In the next lemma, we determine the generators of the tangent space to $S$ at $x_{0}$, namely, the vectors $X^{*} \in T S_{x_{0}}$ for $X$ ranging in the root spaces $\mathfrak{g}^{\mu}$ for $\mu \in \Delta_{\mathfrak{b}}$; see (9-2).

Lemma 9.4. We have the following table.

\begin{tabular}{lll}
\hline$X$ & $\mathfrak{g}^{\mu}$ & $X^{*}$, if $X \in \mathfrak{g}^{\mu}$ \\
\hline$Y_{2 \lambda}$ & $\mathfrak{g}_{\mathfrak{h}}^{2 \lambda}$ & 0 \\
$Y_{\lambda}$ & $\mathfrak{g}_{\mathfrak{h}}^{\lambda}$ & 0 \\
$X_{\lambda}$ & $\mathfrak{g}_{\mathfrak{q}}^{\lambda}$ & $\operatorname{Ad}_{x_{0}} X_{\lambda}$. \\
$B$ & $\mathfrak{b}$ & $i \lambda(B) \operatorname{Ad}_{x_{0}} X_{\lambda}^{0}$ \\
$W_{0}$ & $\mathfrak{g}_{\mathfrak{h} \cap \mathfrak{k}}^{0}$ & $-i \operatorname{Ad}_{x_{0}}\left[X_{\lambda}^{0}, W_{0}\right]$ \\
$Z_{0}$ & $\mathfrak{g}_{\mathfrak{q}}^{0}$ & $\operatorname{Ad}_{x_{0}} Z_{0}$ \\
$Y_{-\lambda}$ & $\mathfrak{g}_{\mathfrak{h}}^{-\lambda}$ & $-i \operatorname{Ad}_{x_{0}}\left[X_{\lambda}^{0}, Y_{-\lambda}\right]$ \\
$X_{-\lambda}$ & $\mathfrak{g}_{\mathfrak{q}}^{-\lambda}$ & $\operatorname{Ad}_{x_{0}} X_{-\lambda}-\frac{1}{2} \operatorname{Ad}_{x_{0}}\left[X_{\lambda}^{0},\left[X_{\lambda}^{0}, X_{-\lambda}\right]\right]$ \\
$Y_{-2 \lambda}$ & $\mathfrak{g}_{\mathfrak{h}}^{-2 \lambda}$ & $-i \operatorname{Ad}_{x_{0}}\left[X_{\lambda}^{0}, Y_{-2 \lambda}\right]+\frac{i}{6} \operatorname{Ad}_{x_{0}}\left[X_{\lambda}^{0},\left[X_{\lambda}^{0},\left[X_{\lambda}^{0}, Y_{-2 \lambda}\right]\right]\right]$ \\
\hline
\end{tabular}

Proof. All rows are obtained by combining the formula $\operatorname{Ad}_{\exp i X} Y=\exp _{\operatorname{ad}_{X}} Y$ with the bracket relations among root vectors. We omit the computations, which are long but straightforward.

Fix $\theta X_{\lambda}^{0} \in \mathfrak{g}_{\mathfrak{q}}^{-\lambda}$, and denote by $\left(\mathfrak{g}_{\mathfrak{q}}^{-\lambda}\right)_{0}$ a complementary subspace to $\mathbb{R} \theta X_{\lambda}^{0}$ in $\mathfrak{g}_{\mathfrak{q}}^{-\lambda}$. By Lemma 9.2(iii) and Lemma 9.4, the tangent space to $S$ at $x_{0}$ is given by $T S_{x_{0}}=T_{\mathbb{C}} S_{x_{0}} \oplus N S_{x_{0}}$, where

(9-4) $T_{\mathbb{C}} S_{x_{0}}=\operatorname{Ad}_{x_{0}}\left(\mathfrak{g}_{\mathfrak{q}}^{0}\right)^{\mathbb{C}} \oplus \operatorname{Ad}_{x_{0}}\left(\mathfrak{g}_{\mathfrak{q}}\right)^{\mathbb{C}} \oplus \operatorname{Ad}_{x_{0}}\left(\mathfrak{g}_{\mathfrak{q}}^{-\lambda}\right)_{0}^{\mathbb{C}} \quad$ and $\quad N S_{x_{0}}=\mathbb{R} \operatorname{Ad}_{x_{0}} \theta X_{\lambda}^{0}$.

Note that if $\Delta_{\mathfrak{b}}$ is reduced, one has $\left(\mathfrak{g}_{\mathfrak{q}}^{-\lambda}\right)_{0}=\{0\}$ by Lemma 9.1(i).

Remark 9.5. There exists a basis of $\mathfrak{g}$ such that the above decomposition of $T S_{x_{0}}$ is orthogonal with respect to the Killing form $B$ of $\mathfrak{g}^{\mathbb{C}}$. If the restricted root system $\Delta_{\mathfrak{b}}$ is nonreduced, one can construct it starting from a basis of $\mathfrak{g}^{\mathbb{C}} / \mathfrak{s}^{\mathbb{C}}$ consisting of root vectors with respect to a maximally split Cartan subalgebra $\mathfrak{s}$ of $\mathfrak{g}$ extending $\mathfrak{b}$. In the reduced case, this is immediate by Lemma 9.1(i).

The Levi form of the G-orbit of $\boldsymbol{x}_{\mathbf{0}}$. The same arguments used in [Geatti 2002, Section 4] yield the following formulas for the Levi form of $S$ at $x_{0}$. Let $Z$ and $W$ be vectors in $T_{\mathbb{C}} S_{x_{0}}$. Then

$$
L_{x_{0}}(Z, W)=\frac{1}{2}\left[(\cdot)^{-1} J W, Z\right]-\frac{i}{2}\left[(\cdot)^{-1} W, Z\right] \bmod \left(T_{\mathbb{C}} S_{x_{0}}\right)^{\mathbb{C}},
$$


where $(\cdot)^{-1} J W$ and $(\cdot)^{-1} W$ are arbitrary elements in the preimages of $J W$ and $W$ by the map defined in (9-1). In the next lemma we compute the Levi form of $S$ at $x_{0}$. Fix $F_{-\lambda}^{0}:=\operatorname{Ad}_{x_{0}} \theta X_{\lambda}^{0}$ as a generator of $N S_{x_{0}}$.

Lemma 9.6. (i) Let $X_{-\lambda} \in\left(\mathfrak{g}_{\mathfrak{q}}^{-\lambda}\right)_{0}$. Set $F_{-\lambda}:=\operatorname{Ad}_{x_{0}} X_{-\lambda}$. Then $L_{x_{0}}\left(F_{-\lambda}, F_{-\lambda}\right)=-\frac{1}{6} \operatorname{Ad}_{x_{0}}\left[\left[X_{\lambda}^{0}, X_{-\lambda}\right], X_{-\lambda}\right]=p F_{-\lambda}^{0} \bmod \left(T_{\mathbb{C}} S_{x_{0}}\right)^{\mathbb{C}}$, where $p \geq 0$.

(ii) Let $Z_{0} \in \mathfrak{g}_{\mathfrak{q}}^{0}$. Write $Z_{0}=\left[X_{\lambda}^{0}, Y_{-\lambda}\right]$ for some $Y_{-\lambda} \in \mathfrak{g}_{\mathfrak{h}}^{-\lambda}$ (see Lemma 9.2), and set $F_{0}:=\operatorname{Ad}_{x_{0}} Z_{0}$. Then

$$
L_{x_{0}}\left(F_{0}, F_{0}\right)=-\frac{1}{2} \operatorname{Ad}_{x_{0}}\left[Y_{-\lambda}, Z_{0}\right]=n F_{-\lambda}^{0} \bmod \left(T_{\mathbb{C}} S_{x_{0}}\right)^{\mathbb{C}}, \text { where } n \leq 0 .
$$

(iii) Let $X_{\lambda} \in \mathfrak{g}_{\mathfrak{q}}^{\lambda}$, and set $F_{\lambda}:=\operatorname{Ad}_{x_{0}} X_{\lambda}$. Then $L_{x_{0}}\left(F_{\lambda}, F_{\lambda}\right)=0$.

(iv) Let $X_{-\lambda} \in\left(\mathfrak{g}_{\mathfrak{q}}^{-\lambda}\right)_{0}$ and $Z_{0} \in \mathfrak{g}_{\mathfrak{q}}^{0}$. Set $F_{-\lambda}:=\operatorname{Ad}_{x_{0}} X_{-\lambda}$ and $F_{0}:=\operatorname{Ad}_{x_{0}} Z_{0}$. Then $L_{x_{0}}\left(F_{-\lambda}, F_{0}\right)=0$.

(v) Let $X_{-\lambda} \in\left(\mathfrak{g}_{\mathfrak{q}}^{-\lambda}\right)_{0}$ and $X_{\lambda} \in \mathfrak{g}_{\mathfrak{q}}^{\lambda}$. Set $F_{-\lambda}:=\operatorname{Ad}_{x_{0}} X_{-\lambda}$ and $F_{\lambda}:=\operatorname{Ad}_{x_{0}} X_{\lambda}$. Then $L_{x_{0}}\left(F_{-\lambda}, F_{\lambda}\right)=a F_{-\lambda}^{0}$, where $a \in \mathbb{C}$.

(vi) Let $X_{\lambda} \in \mathfrak{g}_{\mathfrak{q}}^{\lambda}$ and $Z_{0} \in \mathfrak{g}_{\mathfrak{q}}^{0}$. Set $F_{\lambda}:=\operatorname{Ad}_{x_{0}} X_{\lambda}$ and $F_{0}:=\operatorname{Ad}_{x_{0}} Z_{0}$. Then $L_{x_{0}}\left(F_{0}, F_{\lambda}\right)=0$.

Proof. By way of example, we prove the first two statements. The remaining ones follow similarly, and the details are omitted.

(i) Let $F_{-\lambda}=\operatorname{Ad}_{x_{0}} X_{-\lambda}$. In order to compute the brackets (9-5), we invert the relations in Lemma 9.4 and decompose the results in $\mathfrak{g}^{\mathbb{C}}=\operatorname{Ad}_{x_{0}} \mathfrak{h}^{\mathbb{C}} \oplus \operatorname{Ad}_{x_{0}} \mathfrak{q}^{\mathbb{C}}$. Write $X_{-\lambda}=\left[X_{\lambda}^{0}, Y_{-2 \lambda}\right]$ for some $Y_{-2 \lambda} \in \mathfrak{g}_{\mathfrak{h}}^{2 \lambda}$; see Lemma 9.2. Then

$$
\begin{aligned}
(\cdot)^{-1} J F_{-\lambda}= & -Y_{-2 \lambda}+\frac{1}{6} \operatorname{ad}_{X_{\lambda}^{0}}^{2}\left(Y_{-2 \lambda}\right) \\
= & -\operatorname{Ad}_{x_{0}} Y_{-2 \lambda}+i \operatorname{Ad}_{x_{0}} \operatorname{ad}_{X_{\lambda}^{0}}\left(Y_{-2 \lambda}\right)+\frac{1}{2} \operatorname{Ad}_{x_{0}} \operatorname{ad}_{X_{\lambda}^{0}}^{2}\left(Y_{-2 \lambda}\right) \\
& -\frac{i}{6} \operatorname{Ad}_{x_{0}} \operatorname{ad}_{X_{\lambda}^{0}}^{3}\left(Y_{-2 \lambda}\right)+\frac{3}{8} \operatorname{Ad}_{x_{0}} \operatorname{ad}_{X_{\lambda}^{0}}^{4}\left(Y_{-2 \lambda}\right)
\end{aligned}
$$

and

$$
\begin{aligned}
(\cdot)^{-1} F_{-\lambda} & =\operatorname{ad}_{X_{\lambda}^{0}}\left(Y_{-2 \lambda}\right)+\frac{1}{2} \operatorname{ad}_{X_{\lambda}^{0}}^{3}\left(Y_{-2 \lambda}\right) \\
& =\operatorname{Ad}_{x_{0}} \operatorname{ad}_{X_{\lambda}^{0}}\left(Y_{-2 \lambda}\right)-i \operatorname{Ad}_{x_{0}} \operatorname{ad}_{X_{\lambda}^{0}}^{2}\left(Y_{-2 \lambda}\right)+\frac{i}{6} \operatorname{Ad}_{x_{0}} \operatorname{ad}_{X_{\lambda}^{0}}^{4}\left(Y_{-2 \lambda}\right) .
\end{aligned}
$$

By formulas (9-5), we obtain

$$
\begin{aligned}
L_{x_{0}}\left(F_{-\lambda}, F_{-\lambda}\right) & =-\frac{1}{6} \operatorname{Ad}_{x_{0}}\left[\operatorname{ad}_{X_{\lambda}^{0}}^{2}\left(Y_{-2 \lambda}\right), \operatorname{ad}_{X_{\lambda}^{0}}\left(Y_{-2 \lambda}\right)\right] \\
& =-\frac{1}{6} \operatorname{Ad}_{x_{0}}\left[\left[X_{\lambda}^{0}, X_{-\lambda}\right], X_{-\lambda}\right] \bmod \left(T_{\mathbb{C}} S_{x_{0}}\right)^{\mathbb{C}} .
\end{aligned}
$$


To complete the proof of the statement, set $F_{\lambda}^{0}:=\operatorname{Ad}_{x_{0}} X_{\lambda}^{0}$, and note that due to Remark 9.5, the component $p F_{-\lambda}^{0}$ of the above brackets in $N S_{x_{0}}$ is given by

$$
B\left(L_{x_{0}}\left(F_{-\lambda}, F_{-\lambda}\right), F_{\lambda}^{0}\right)=p B\left(F_{-\lambda}^{0}, F_{\lambda}^{0}\right) .
$$

Since $B\left(F_{-\lambda}^{0}, F_{\lambda}^{0}\right)=B\left(X_{-\lambda}^{0}, \theta X_{-\lambda}^{0}\right)$ is negative, the real number $p$ has the same sign as

$$
B\left(\left[\left[X_{\lambda}^{0}, X_{-\lambda}\right], X_{-\lambda}\right], X_{\lambda}^{0}\right)=-B\left(\left[X_{\lambda}^{0}, X_{-\lambda}\right],\left[X_{\lambda}^{0}, X_{-\lambda}\right]\right) .
$$

By Lemmas 9.1 and 9.2, the brackets $\left[X_{\lambda}^{0}, X_{-\lambda}\right]$ lie in $\mathfrak{k}$, so

$$
B\left(\left[X_{\lambda}^{0}, X_{-\lambda}\right],\left[X_{\lambda}^{0}, X_{-\lambda}\right]\right)
$$

is nonpositive. It follows that $p \geq 0$, as claimed.

(ii) Write $Z_{0}=\left[X_{\lambda}^{0}, Y_{-\lambda}\right]$ for some $Y_{-\lambda} \in \mathfrak{g}_{\mathfrak{h}}^{-\lambda}$; see Lemmas 9.1 and 9.2. By computations similar to the above ones, we have

$$
\begin{aligned}
& (\cdot)^{-1} J F_{0}=-Y_{-\lambda}=-\operatorname{Ad}_{x_{0}} Y_{-\lambda}+i \operatorname{Ad}_{x_{0}} \operatorname{ad}_{X_{\lambda}^{0}}\left(Y_{-\lambda}\right)+\frac{1}{2} \operatorname{Ad}_{x_{0}} \operatorname{ad}_{X_{\lambda}^{0}}^{2}\left(Y_{-\lambda}\right), \\
& (\cdot)^{-1} F_{0}=Z_{0}=\operatorname{Ad}_{x_{0}} Z_{0}-i \operatorname{Ad}_{x_{0}} \operatorname{ad}_{X_{\lambda}^{0}}\left(Z_{0}\right)
\end{aligned}
$$

and

$$
\left.L_{x_{0}}\left(F_{0}, F_{0}\right)=-\frac{1}{2} \operatorname{Ad}_{x_{0}}\left[Y_{-\lambda},\left[X_{\lambda}^{0}, Y_{-\lambda}\right]\right]=-\frac{1}{2} \operatorname{Ad}_{x_{0}}\left[Y_{-\lambda}, Z_{0}\right] \bmod T_{\mathbb{C}} S_{x_{0}}\right)^{\mathbb{C}} .
$$

To complete the proof of (ii), observe that $n=B\left(L\left(F_{0}, F_{0}\right), F_{\lambda}^{0}\right) / B\left(F_{-\lambda}^{0}, F_{\lambda}^{0}\right)$ has the same sign as $B\left(\left[Y_{-\lambda},\left[X_{\lambda}^{0}, Y_{-\lambda}\right]\right], X_{\lambda}^{0}\right)=B\left(\left[X_{\lambda}^{0}, Y_{-\lambda}\right],\left[X_{\lambda}^{0}, Y_{-\lambda}\right]\right)$. Since $\left[X_{\lambda}^{0}, Y_{-\lambda}\right]$ lies in $\mathfrak{k}$, the above expression is nonpositive and $n \leq 0$, as claimed.

Proposition 9.7. Let $S$ be the $G$-orbit of the point $x_{0}=\exp i X_{\lambda}^{0} \cdot z$.

If the restricted root system $\Delta_{\mathfrak{b}}$ is reduced, then the Levi form of the orbit $S$ is definite provided that $\operatorname{dim} G / K>2$. It is identically zero if $\operatorname{dim} G / K=2$.

If the restricted root system $\Delta_{\mathfrak{b}}$ is nonreduced, then the Levi form of the orbit $S$ is indefinite.

Proof. If the restricted root system $\Delta_{\mathfrak{b}}$ is reduced, then only (ii), (iii), and (iv) of Lemma 9.6 apply. By Lemma 9.6(ii), for every $F_{0} \in \operatorname{Ad}_{x_{0}}\left(\mathfrak{g}_{\mathfrak{q}}^{0}\right)^{\mathbb{C}}$, the real numbers $B\left(L\left(F_{0}, F_{0}\right), F_{\lambda}^{0}\right)$ all have the same sign. In other words, the restriction of the Levi form to $\operatorname{Ad}_{x_{0}}\left(\mathfrak{g}_{\mathfrak{q}}^{0}\right)^{\mathbb{C}} \subset T_{\mathbb{C}} S_{x_{0}}$ is either definite or identically zero. It is identically zero when $\operatorname{ad}_{X_{\lambda}^{0}}: \mathfrak{g}_{\mathfrak{h}}^{-\lambda} \rightarrow \mathfrak{g}_{\mathfrak{q}}^{0}$ is the zero-map. This happens if and only if $\mathfrak{g}=\mathfrak{s l}(2, \mathbb{R})$ and $\operatorname{dim} G / K=2$.

If the restricted root system $\Delta_{\mathfrak{b}}$ is nonreduced, then $\operatorname{dim} G / K>2$. In this case, the restriction of the Levi form to $\operatorname{Ad}_{x_{0}}\left(\mathfrak{g}_{\mathfrak{q}}^{0}\right)^{\mathbb{C}} \subset T_{\mathbb{C}} S_{x_{0}}$ is definite. Also, by Lemma 9.6(i) and Lemma 9.2(iii), the restriction of the Levi form to $\operatorname{Ad}_{x_{0}}\left(\mathfrak{g}_{\mathfrak{q}}^{0}\right)^{\mathbb{C}} \subset T_{\mathbb{C}} S_{x_{0}}$ is definite with opposite sign. As a result, the Levi form of $S$ is indefinite. 
The Levi form of the G-orbit $y_{0}$. By the same methods, one can compute the tangent space and the Levi form of the $G$-orbit $S$ of the point $y_{0}=\exp \left(-i X_{\lambda}^{0}\right) \cdot z$. As we already remarked, the orbits $G \cdot x_{0}$ and $G \cdot y_{0}$ are distinct only when the restricted root system of $\mathfrak{g}$ is reduced. So we focus on this case. For the tangent space to $S$ at $y_{0}$, one has $T S_{y_{0}}=T_{\mathbb{C}} S_{y_{0}} \oplus N S_{y_{0}}$, where

$$
T_{\mathbb{C}} S_{y_{0}}=\operatorname{Ad}_{y_{0}}\left(\mathfrak{g}_{\mathfrak{q}}^{0}\right)^{\mathbb{C}} \oplus \operatorname{Ad}_{y_{0}}\left(\mathfrak{g}_{\mathfrak{q}}^{\lambda}\right)^{\mathbb{C}} \quad \text { and } \quad N S_{y_{0}}=\mathbb{R} \operatorname{Ad}_{y_{0}} \theta X_{\lambda}^{0} .
$$

Fix $F_{-\lambda}^{0}:=\operatorname{Ad}_{y_{0}} \theta X_{\lambda}^{0}$ as a generator of $N S_{y_{0}}$. For the Levi form, one has the following results.

Lemma 9.8. (i) Let $Z_{0} \in \mathfrak{g}_{\mathfrak{q}}^{0}$. Write $Z_{0}=\left[X_{\lambda}^{0}, Y_{-\lambda}\right]$, for some $Y_{-\lambda} \in \mathfrak{g}_{\mathfrak{h}}^{-\lambda}$ (see Lemma 9.2), and set $F_{0}:=\operatorname{Ad}_{y_{0}} Z_{0}$. Then

$L_{y_{0}}\left(F_{0}, F_{0}\right)=\frac{1}{2} \operatorname{Ad}_{y_{0}}\left[Y_{-\lambda}, Z_{0}\right]=s F_{-\lambda}^{0} \quad \bmod \left(T_{\mathbb{C}} S_{y_{0}}\right)^{\mathbb{C}}, \quad$ where $s \geq 0$.

(ii) Let $X_{\lambda} \in \mathfrak{g}_{\mathfrak{q}}^{\lambda}$, and set $F_{\lambda}:=\operatorname{Ad}_{y_{0}} X_{\lambda}$. Then $L_{y_{0}}\left(F_{\lambda}, F_{\lambda}\right)=0$.

(iii) Let $Z_{0} \in \mathfrak{g}_{\mathfrak{q}}^{0}$ and $X_{\lambda} \in \mathfrak{g}_{\mathfrak{q}}^{\lambda}$. Set $F_{0}:=\operatorname{Ad}_{y_{0}} Z_{0}$ and $F_{\lambda}:=\operatorname{Ad}_{y_{0}} X_{\lambda}$. Then $L_{y_{0}}\left(F_{0}, F_{\lambda}\right)=0$.

Proposition 9.9. Let $S$ be the $G$-orbit of the point $y_{0}$.

If the restricted root system $\Delta_{\mathfrak{b}}$ is reduced, then the Levi form of the orbit $S$ is definite provided that $\operatorname{dim} G / K>2$. It is identically zero if $\operatorname{dim} G / K=2$.

Remark 9.10. By Propositions 9.7 and 9.9, if the restricted root system $\Delta_{\mathfrak{b}}$ is reduced, then the Levi form of the orbits represented by $w_{1}$ and $w_{2}$ in diagram (4-4) is definite. This is consistent with the fact that these orbits lie in the boundary of the Stein domains $D_{1}(0)$ and $D_{2}(0)$, respectively; see Theorem 6.1. If $\operatorname{dim} G / K=2$, all orbits represented by $w_{1}, \ldots, w_{4}$ in diagram (4-3) are Levi flat. We refer to Example 6.3 for a classification of $G$-invariant Stein domains bounded by such orbits. If the restricted root system $\Delta_{\mathfrak{b}}$ is nonreduced, then the Levi form of the orbit represented by $w_{5}$ in diagrams (4-9) and (4-10) is indefinite. As a consequence, this orbit cannot lie in the boundary of a Stein $G$-invariant domain in $G^{\mathbb{C}} / K^{\mathbb{C}}$.

9.2. Nonclosed orbits with a $\boldsymbol{C R}$ singular orbit in their closure. We consider now nonclosed $G$-orbits containing in their closure the orbit of a point $z=g K^{\mathbb{C}}=$ $\exp i A K^{\mathbb{C}} \in A_{0}$, satisfying the condition $\alpha(A)=\pi / 4$, with $\alpha$ a simple restricted root; see (4-7). In this case the singular orbit $G \cdot z$ has dimension greater than $\operatorname{dim} G / K$. Recall from Section 4.2 that the isotropy subgroup $H^{\prime}$ of $z$ in $G$ is contained in $G^{\prime}:=Z_{G}\left(g^{4}\right)$ and that $G^{\prime} / H^{\prime}$ is diffeomorphic to a rank-one, pseudoRiemannian symmetric space, totally real in $G^{\mathbb{C}} / K^{\mathbb{C}}$. Let $\left(\mathfrak{g}^{\prime}=\mathfrak{h}^{\prime} \oplus \mathfrak{q}^{\prime}, \tau_{z}\right)$ be the associated symmetric algebra. Nonclosed $G$-orbits in $G^{\mathbb{C}} / K^{\mathbb{C}}$ containing $G \cdot z$ in their closure are in one-to-one correspondence with the nilpotent $\mathrm{Ad}_{\mathrm{H}^{\prime}}$-orbits in $\mathfrak{q}^{\prime}$; see (4-1) and Remark 4.2. 
To explicitly determine reference points for such nonclosed orbits and their tangent spaces, we decompose $\mathfrak{g}$ and $\mathfrak{g}^{\prime}$ by an appropriate restricted root system. Let $\mathscr{b}^{\prime}=\exp i \mathfrak{c}^{\prime} \cdot z$ be the standard Cartan subset with base point $z$. Recall that $\mathfrak{c}^{\prime}=\mathbb{R}(X+\theta(X))$, where $X$ is a nonzero vector in $\mathfrak{g}^{2 \alpha}$. In particular, $\mathfrak{c}^{\prime}$ is contained in $\mathfrak{g}^{\prime}$; see (4-8). Define $\mathfrak{b}^{\prime}=\mathbb{R}(X-\theta(X))$. Then $\mathfrak{b}^{\prime}$ is a maximal abelian subalgebra in $\mathfrak{h}^{\prime} \cap \mathfrak{p}$ and the restricted root decompositions of $\mathfrak{g}$ with respect to $\mathfrak{b}^{\prime}$ is given by

$$
\mathfrak{g}=Z_{\mathfrak{k}}\left(\mathfrak{b}^{\prime}\right) \oplus \mathfrak{b}^{\prime} \oplus \mathfrak{g}^{ \pm 2 \lambda} \oplus \mathfrak{g}^{ \pm \lambda}
$$

In order to determine how the above root decomposition restricts to the subalgebra $\mathfrak{g}^{\prime}$, observe that in general $\mathfrak{g}^{\prime}$ is not simple, but is the direct sum of a copy of $\mathfrak{s o}(m, 1)$ with $m=\operatorname{dim} \mathfrak{g}^{2 \alpha}+1$ (even) and a compact subalgebra $\mathfrak{l}$ entirely contained in $\mathfrak{h}^{\prime}$, that is,

$$
\mathfrak{g}^{\prime}=\mathfrak{l} \oplus \mathfrak{s o}(m, 1) \quad \text { and } \quad \mathfrak{h}^{\prime}=\mathfrak{l} \oplus \mathfrak{s o}(m-1,1) .
$$

Observe also that all real rank one Lie algebras with a nonreduced restricted root system are equal-rank. Hence the root system $\Delta$ of $\mathfrak{g}^{\mathbb{C}}$ with respect to a maximally split Cartan subalgebra of $\mathfrak{g}$ extending $\mathfrak{b}^{\prime}$ contains a real root. Since $\mathfrak{g}^{2 \lambda}$ is odddimensional (see Table 4.0), the restriction of this real root to $\mathfrak{b}^{\prime}$ coincides the restricted root $2 \lambda$; see [Helgason 1978, page 584]. Since $\mathfrak{g}^{\prime}$ has a reduced restricted root system (see (4-8)) and because $\mathfrak{s o}(m, 1)$ with $m$ even is equal-rank, we have $\mathfrak{g}^{\prime} \cap \mathfrak{g}^{2 \lambda} \neq\{0\}$. It follows that the restricted root decomposition of $\mathfrak{g}^{\prime}$ with respect to $\mathfrak{b}^{\prime}$ is given by

$$
\mathfrak{g}^{\prime}=Z_{\mathfrak{k}}\left(\mathfrak{b}^{\prime}\right) \oplus \mathfrak{b}^{\prime} \oplus \mathfrak{g}^{ \pm 2 \lambda}
$$

Let

$$
\mathfrak{g}^{\prime}=\mathfrak{h}^{\prime} \oplus \mathfrak{q}^{\prime}, \quad \text { with } \mathfrak{h}^{\prime}=\mathfrak{g}_{\mathfrak{h}^{\prime} \cap \mathfrak{k}}^{0} \oplus \mathfrak{g}_{\mathfrak{h}^{\prime}}^{ \pm 2 \lambda} \oplus \mathfrak{b}^{\prime} \quad \text { and } \quad \mathfrak{q}^{\prime}=\mathfrak{g}_{\mathfrak{q}^{\prime}}^{0} \oplus \mathfrak{g}_{\mathfrak{q}^{\prime}}^{ \pm 2 \lambda}
$$

be the combined decomposition of $\mathfrak{g}^{\prime}$. Note that $\mathfrak{g}^{\prime}$ has real rank one as well. Therefore $\mathfrak{g}_{\mathfrak{q}^{\prime}}^{0} \subset \mathfrak{k}$ and an analogue of Lemma 9.1 holds for $\mathfrak{g}^{\prime}$. Set $\mathfrak{g}[\lambda]:=\mathfrak{g}^{\lambda} \oplus \mathfrak{g}^{-\lambda}$ and $\mathfrak{g}[\alpha]:=\mathfrak{g}^{\alpha} \oplus \mathfrak{g}^{-\alpha}$ (here $\alpha$ is a restricted root in $\Delta_{\mathfrak{a}}$, as in Section 4).

Lemma 9.11. The following facts hold:

(i) $\operatorname{dim} \mathfrak{g}_{\mathfrak{q}^{\prime}}^{ \pm 2 \lambda}=1$.

(ii) $\left[\mathfrak{g}_{\mathfrak{q}^{\prime}}^{2 \lambda}, \mathfrak{g}_{\mathfrak{h}^{\prime}}^{-2 \lambda}\right]=\mathfrak{g}_{\mathfrak{q}^{\prime}}^{0}$ and $\left[\mathfrak{g}_{\mathfrak{q}^{\prime}}^{-2 \lambda}, \mathfrak{g}_{\mathfrak{h}^{\prime}}^{2 \lambda}\right]=\mathfrak{g}_{\mathfrak{q}^{\prime}}^{0}$.

(iii) the decomposition $\mathfrak{g}=\mathfrak{g}^{\prime} \oplus \mathfrak{g}[\alpha]$ is $\operatorname{ad}_{\mathfrak{b}^{\prime}}$-stable. In particular $\mathfrak{g}[\alpha]=\mathfrak{g}[\lambda]$.

Proof. Statement (i) follows from the fact that $\operatorname{dim} \mathfrak{q}^{\prime} \cap \mathfrak{p}=1$ (see the proof of Lemma 4.5(ii), while (ii) can be checked directly. 
To prove (iii), note that $\operatorname{ad}_{\mathfrak{b}^{\prime}} \mathfrak{g}^{\prime} \subset \mathfrak{g}^{\prime}$. Moreover, $\operatorname{ad}_{\mathfrak{b}^{\prime}}\left(\mathfrak{g}^{\alpha} \oplus \mathfrak{g}^{-\alpha}\right) \subset\left(\mathfrak{g}^{\alpha} \oplus \mathfrak{g}^{-\alpha}\right)$. By (4-8) and (9-6) it follows that the decomposition $\mathfrak{g}=\mathfrak{g}^{\prime} \oplus \mathfrak{g}[\alpha]$ is ad $\operatorname{ad}_{\mathfrak{b}^{\prime}}$-stable and $\mathfrak{g}[\alpha]=\mathfrak{g}[\lambda]$.

Reference points for nonclosed G-orbits. Reference points for nonclosed orbits containing $G \cdot z$ in their closures can be obtained by applying the methods of the previous section to the symmetric space $G^{\prime} / H^{\prime}$; see (9-3). In this case take $X \in \mathfrak{g}^{2 \alpha}$, $\theta X$ and $A:=[\theta X, X]$, normalized so that $2 \alpha(A)=2$. Then

$$
X_{2 \lambda}^{0}=\frac{1}{2}(A-(X+\theta X)) \quad \text { and } \quad X_{-2 \lambda}^{0}=\frac{1}{2}(A+(X+\theta X))
$$

are generators of $\mathfrak{g}_{\mathfrak{q}^{\prime}}^{2 \lambda}$ and $\mathfrak{g}_{\mathfrak{q}^{\prime}}^{-2 \lambda}$, respectively, and the points

$$
\begin{array}{ll}
x_{0}=\exp i X_{2 \lambda}^{0} \cdot z, & x_{1}=\exp i X_{-2 \lambda}^{0} \cdot z, \\
y_{0}=\exp \left(-i X_{2 \lambda}^{0}\right) \cdot z, & y_{1}=\exp \left(-i X_{-2 \lambda}^{0}\right) \cdot z
\end{array}
$$

lie on nonclosed $G$-orbits in $G^{\mathbb{C}} / K^{\mathbb{C}}$ containing the singular orbit $G \cdot z$ in their closures. If the orbit diagram is of type (4-9), there are four such orbits, represented by $w_{3}, w_{2}, w_{1}$ and $w_{4}$, respectively. If the orbit diagram is of type (4-10), the points $x_{0}$ and $x_{1}$ lie on the same $G$-orbit, represented by $w_{2}$. Similarly, the points $y_{0}$ and $y_{1}$ lie on the same $G$-orbit represented by $w_{1}$. The $G$-orbits of $y_{0}$ and $y_{1}$ lie on the boundary of the Stein domain $D_{1}(0)$; see Theorem 6.1 .

The tangent space to the $G$-orbit of $x_{0}$. Denote by $S$ the $G$-orbit of the point $x_{0}=\exp i X_{2 \lambda}^{0} \cdot z$. To compute the tangent space $T S_{x_{0}}$, observe that at the point $z$

$$
T(G \cdot z)_{z}=\mathfrak{q}^{\prime} \oplus V_{z}, \quad \text { and } \quad T\left(G^{\mathbb{C}} / K^{\mathbb{C}}\right)_{z}=\operatorname{Ad}_{z} \mathfrak{p}^{\mathbb{C}}=\left(\mathfrak{q}^{\prime}\right)^{\mathbb{C}} \oplus V_{z},
$$

where $\mathfrak{q}^{\prime}=T\left(G^{\prime} \cdot z\right)_{z}$ and $V_{z}=\operatorname{Ad}_{z} \mathfrak{g}[\alpha]_{\mathfrak{p}}^{\mathbb{C}}$ is a complex subspace of $\mathfrak{g}[\alpha]^{\mathbb{C}}$; see [Geatti 2002, Proposition 3.2]. It follows that

$$
T S_{x_{0}} \subset \operatorname{Ad}_{x_{0}}\left(\mathfrak{q}^{\prime}\right)^{\mathbb{C}} \oplus \operatorname{Ad}_{x_{0}} V_{z}
$$

To determine generators for $T S_{x_{0}}$, fix a maximally split Cartan subalgebra $\mathfrak{s}$ of $\mathfrak{g}$ extending $\mathfrak{b}^{\prime}$ and entirely contained in $\mathfrak{h}^{\prime}$ (one can check that in all cases under consideration $\mathfrak{h}^{\prime}$ has the same rank as $\mathfrak{g}$ and such a Cartan subalgebra indeed exists). Let

$$
\mathfrak{g}^{\mathbb{C}}=\mathfrak{s}^{\mathbb{C}} \bigoplus_{\beta \in \Delta} \mathfrak{g}^{\beta}
$$

be the corresponding root decomposition of $\mathfrak{g}^{\mathbb{C}}$, and let $\left\{Z_{\beta}\right\}_{\beta \in \Delta}$ be a complex basis of $\mathfrak{g}^{\mathbb{C}} / \mathfrak{s}^{\mathbb{C}}$ consisting of root vectors $Z_{\beta} \in \mathfrak{g}^{\beta}$. Choose compatible orderings of $\Delta_{\mathfrak{b}^{\prime}}$ and $\Delta$ (that is, a root $\beta \in \Delta$ is positive if its restriction to $\mathfrak{b}^{\prime}$ is). Fix $\lambda \in \Delta_{\mathfrak{b}^{\prime}}$ (either a positive or a negative short restricted root), and denote by $\Delta_{\lambda}$ the set of 
roots in $\Delta$ that, when restricted to $\mathfrak{b}^{\prime}$, are equal to $\lambda$. The set $\Delta_{\lambda}$ consists of pairs of complex roots

$$
\beta_{1}, \bar{\beta}_{1}, \ldots, \beta_{m}, \bar{\beta}_{m}, \quad \text { where } m=\frac{1}{2} \operatorname{dim} \mathfrak{g}^{\lambda},
$$

all with the same real part, equal to $\lambda$. For $\beta_{i}, \bar{\beta}_{i} \in \Delta_{\lambda}$, choose root vectors $Z_{\beta_{i}} \in \mathfrak{g}^{\beta_{i}}$ and $\sigma Z_{\beta_{i}} \in \mathfrak{g}^{\bar{\beta}_{i}}$. Then the vectors defined as

$$
X_{\lambda}^{i}=Z_{\beta_{i}}+\sigma Z_{\beta_{i}} \quad \text { and } \quad Y_{\lambda}^{i}=-i\left(Z_{\beta_{i}}-\sigma Z_{\beta_{i}}\right) \quad \text { for } i=1, \ldots, m,
$$

belong to $\mathfrak{g}$ and form a basis of the restricted root space $\mathfrak{g}^{\lambda}$.

Lemma 9.12. The following facts hold:

(i) For all $i=1, \ldots, m$, one has $\tau_{z} Z_{\beta_{i}}=-Z_{\beta_{i}}$ and $i \tau_{z} X_{\lambda}^{i}=Y_{\lambda}^{i}$.

(ii) For every $i=1, \ldots, m$, the brackets $\left[X_{\lambda}^{i}, i \tau_{z} X_{\lambda}^{i}\right]$ lie in $\mathfrak{g}_{\mathfrak{q}^{\prime}}^{2 \lambda}$. For at least one index $i$, such brackets are nonzero.

(iii) For all $i, j=1, \ldots, m$ with $i \neq j$, the brackets $\left[X_{\lambda}^{i}, i \tau_{z} X_{\lambda}^{j}\right]$ have no components in $\mathfrak{g}_{\mathfrak{q}^{\prime}}^{2 \lambda}$.

Proof. (i) Since the Cartan subalgebra $\mathfrak{s}$ lies in $\mathfrak{h}^{\prime}$, it is pointwise fixed by $\tau_{z}$. As a consequence, all root spaces $\mathfrak{g}^{\beta}$ with $\beta \in \Delta$ are $\tau_{z}$-stable. The inclusion $V_{z} \subset \operatorname{Ad}_{z} \mathfrak{p}^{\mathbb{C}}$ (see (9-8)) implies that $\tau_{z} Z_{\beta_{i}}=-Z_{\beta_{i}}$ for $i=1, \ldots, m$. Since $\sigma \tau_{z}=-\tau_{z} \sigma$ on $V_{z} \subset \mathfrak{g}[\alpha]^{\mathbb{C}}$, one has $i \tau_{z} X_{\lambda}^{i}=Y_{\lambda}^{i}$, as desired.

(ii) By the definitions of $X_{\lambda}^{i}$ and $Y_{\lambda}^{i}$, one has

$$
\left[X_{\lambda}^{i}, i \tau_{z} X_{\lambda}^{i}\right]=\left[X_{\lambda}^{i}, Y_{\lambda}^{i}\right]=2 i\left[Z_{\beta_{i}}, \sigma Z_{\beta_{i}}\right] \in \mathfrak{g}^{2 \lambda} .
$$

By (i) and the fact that $\tau_{z} \sigma=-\sigma \tau_{z}$ on $\mathfrak{g}[\lambda]^{\mathbb{C}}=\mathfrak{g}[\alpha]^{\mathbb{C}}$, one also has

$$
\tau_{z}\left(2 i\left[Z_{\beta_{i}}, \sigma Z_{\beta_{i}}\right]\right)=-2 i\left[Z_{\beta_{i}}, \sigma Z_{\beta_{i}}\right] .
$$

This implies that $\left[X_{\lambda}^{i}, i \tau_{z} X_{\lambda}^{i}\right]$ lies in $\mathfrak{g}_{\mathfrak{q}^{\prime}}^{2 \lambda}$, as claimed. To prove the second part (ii), consider the set $\Delta_{2 \lambda}$ consisting of the roots in $\Delta$ that, when restricted to $\mathfrak{b}^{\prime}$, coincide with $2 \lambda$. Since $\Delta_{2 \lambda}$ contains a real root in $\Delta$ and such a root is not simple (see Satake diagrams in [Helgason 1978, page 532]), there exist $\beta, \bar{\beta} \in \Delta_{\lambda}$ such that $\beta+\bar{\beta}=2 \lambda$. This shows that at least one of the brackets $\left[X_{\lambda}^{i}, i \tau_{z} X_{\lambda}^{i}\right]$ has a nonzero component in $\mathfrak{g}^{2 \lambda}$.

(iii) Let $\beta_{i}, \beta_{j}$ be roots in $\Delta_{\lambda}$, with $\beta_{j} \neq \beta_{i}, \bar{\beta}_{i}$. If either $\beta_{i}+\beta_{j}$ or $\beta_{i}+\bar{\beta}_{j}$ is a root in $\Delta$, then it is a root in $\Delta_{2 \lambda}$, with nonzero imaginary part. Since the root spaces relative to the real root in $\Delta_{2 \lambda}$ are contained in $\left(\mathfrak{g}_{\mathfrak{q}^{\prime}}^{2 \lambda}\right)^{\mathbb{C}}$ and $\operatorname{dim}\left(\mathfrak{g}_{\mathfrak{q}^{\prime}}^{2 \lambda}\right)^{\mathbb{C}}=1$ (see Lemma 9.2), root spaces relative to the remaining roots in $\Delta_{2 \lambda}$ are necessarily contained in $\left(\mathfrak{g}_{\mathfrak{h}^{\prime}}^{2 \lambda} \mathbb{C}^{\mathbb{C}}\right.$. Hence the statement follows. 
For $\lambda \in \Delta_{\mathfrak{b}^{\prime}}^{+}$, fix bases of $\mathfrak{g}^{\lambda}$ and $\mathfrak{g}^{-\lambda}$ of the form

$$
X_{\lambda}^{1}, i \tau_{z} X_{\lambda}^{1}, \ldots, X_{\lambda}^{m}, i \tau_{z} X_{\lambda}^{m} \quad \text { and } \quad X_{-\lambda}^{1}, i \tau_{z} X_{-\lambda}^{1}, \ldots, X_{-\lambda}^{m}, i \tau_{z} X_{-\lambda}^{m},
$$

respectively. For $i, j=1, \ldots, m$, define

$$
w_{i}:=\frac{1}{2} \operatorname{Ad}_{x_{0}}\left(X_{\lambda}^{i}-\tau_{z} X_{\lambda}^{i}\right) \quad \text { and } \quad v_{j}:=\frac{1}{2} \operatorname{Ad}_{x_{0}}\left(X_{-\lambda}^{j}-\tau_{z} X_{-\lambda}^{j}\right) .
$$

In the next lemma, we compute the images of the vectors in (9-10) under the map $*: \mathfrak{g} \rightarrow T S_{x_{0}}$ defined in (9-1). We omit the straightforward proof.

Lemma 9.13. The images of the vectors in (9-10) under the map (9-1) are

(i) $\left(X_{\lambda}^{i}\right)^{*}=w_{i}$;

(ii) $\left(i \tau_{z} X_{\lambda}^{i}\right)^{*}=-i w_{i}$;

(iii) $\left(X_{-\lambda}^{j}\right)^{*}=v_{j}-i w^{\prime}$, where $w^{\prime}=\operatorname{Ad}_{x_{0}}\left[X_{2 \lambda}^{0}, X_{-\lambda}^{j}\right]$; and

(iv) $\left(i \tau_{z} X_{-\lambda}^{j}\right)^{*}=-i v_{j}-i w^{\prime \prime}$, where $w^{\prime \prime}=\operatorname{Ad}_{x_{0}}\left[X_{2 \lambda}^{0}, i \tau_{z} X_{-\lambda}^{j}\right]$.

Let $W_{x_{0}}^{+}$be the complex subspace of $W_{x_{0}}$ spanned by the vectors $\left\{w_{1}, \ldots, w_{m}\right\}$, and let $W_{x_{0}}^{-}$be the one spanned by $\left\{v_{1}, \ldots, v_{m}\right\}$. By (9-9), the results of Section 9.1 applied to the symmetric space $G^{\prime} / H^{\prime}$ and Lemma 9.13, the tangent space to $S$ at $x_{0}$ is given by $T S_{x_{0}}=T_{\mathbb{C}} S_{x_{0}} \oplus N S_{x_{0}}$, where

$$
T_{\mathbb{C}} S_{x_{0}}=T_{\mathbb{C}}\left(G^{\prime} \cdot x_{0}\right)_{x_{0}} \oplus W_{x_{0}}^{+} \oplus W_{x_{0}}^{-} \quad \text { and } \quad N S_{x_{0}}=\mathbb{R} \operatorname{Ad}_{x_{0}} \theta X_{2 \lambda}^{0} .
$$

Fix $F_{-2 \lambda}^{0}:=\operatorname{Ad}_{x_{0}} \theta X_{2 \lambda}^{0}$ as a generator of $N S_{x_{0}}$.

Lemma 9.14. The following facts hold.

(i) The decomposition of $T_{\mathbb{C}} S_{x_{0}}$ given in (9-11) is orthogonal with respect to the Levi form.

(ii) Let $W \in W_{x_{0}}^{+}$. Then $L_{x_{0}}(W, W)=0$.

(iii) Let $W \in W_{x_{0}}^{-}$. Then $L_{x_{0}}(W, W)=b F_{-2 \lambda}^{0}$, with $b \geq 0$.

(iv) Let $Z \in T_{\mathbb{C}}\left(G^{\prime} \cdot x_{0}\right)_{x_{0}}$. Then $L_{x_{0}}(Z, Z)=n F_{-2 \lambda}^{0}$, with $n \leq 0$.

Proof. (i) Let $Z \in T_{\mathbb{C}}\left(G^{\prime} \cdot x_{0}\right)_{x_{0}}$ and $W \in W_{x_{0}}$. To show that $L(Z, W) \equiv L(W, Z) \equiv 0$, observe that both $(\cdot)^{-1} J Z$ and $(\cdot)^{-1} Z$ belong to $\mathfrak{g}^{\prime}=\mathfrak{h}^{\prime} \oplus \mathfrak{q}^{\prime}$ and can be written

$$
\begin{gathered}
(\cdot)^{-1} J Z=\operatorname{Ad}_{x_{0}} X_{0}+\operatorname{Ad}_{x_{0}} X_{2 \lambda}+\operatorname{Ad}_{x_{0}} X_{-2 \lambda}, \\
(\cdot)^{-1} Z=\operatorname{Ad}_{x_{0}} Y_{0}+\operatorname{Ad}_{x_{0}} Y_{2 \lambda}+\operatorname{Ad}_{x_{0}} Y_{-2 \lambda},
\end{gathered}
$$

according to the $\operatorname{ad}_{\mathfrak{b}^{\prime}}$-root decomposition of $\mathfrak{g}^{\prime}$ given in (9-6). Similarly, by (9-8), the vector $W \in W_{x_{0}}^{+} \oplus W_{x_{0}}^{+}=\operatorname{Ad}_{x_{0}} \operatorname{Ad}_{z} \mathfrak{g}[\lambda]_{\mathfrak{p}}^{\mathbb{C}}$ can be written as

$$
W=\operatorname{Ad}_{x_{0}} \operatorname{Ad}_{z} P_{\lambda}+i \operatorname{Ad}_{x_{0}} \operatorname{Ad}_{z} Q_{\lambda},
$$


where

$\operatorname{Ad}_{z} P_{\lambda}=U_{\lambda}+i V_{-\lambda}-\theta U_{\lambda}+i \theta V_{-\lambda} \quad$ and $\quad \operatorname{Ad}_{z} Q_{\lambda}=U_{\lambda}^{\prime}+i V_{-\lambda}^{\prime}-\theta U_{\lambda}^{\prime}+i \theta V_{-\lambda}^{\prime}$ with $U_{\lambda}, U_{\lambda}^{\prime} \in \mathfrak{g}^{\lambda}$ and $V_{-\lambda}, V_{-\lambda}^{\prime} \in \mathfrak{g}^{-\lambda}$. One can verify that none of the brackets in (9-5) has a component in $\operatorname{Ad}_{x_{0}} \mathfrak{g}_{\mathfrak{q}^{\prime}}^{-2 \lambda}$, and $L_{x_{0}}(Z, W) \equiv 0$, as required.

Let $w_{i} \in W_{x_{0}}^{+}$and $v_{j} \in W_{x_{0}}^{-}$. Then, modulo $\left(T_{\mathbb{C}} S_{x_{0}}\right)^{\mathbb{C}}$, the Levi form is given by $2 L_{x_{0}}\left(w_{i}, v_{j}\right) \equiv-\frac{1}{2} \operatorname{Ad}_{x_{0}}\left[i \tau_{z} X_{\lambda}^{i},\left(X_{-\lambda}^{j}-\tau_{z} X_{-\lambda}^{j}\right)\right]-\frac{i}{2} \operatorname{Ad}_{x_{0}}\left[X_{\lambda}^{i},\left(X_{-\lambda}^{j}-\tau_{z} X_{-\lambda}^{j}\right)\right]$.

In particular, $L_{x_{0}}\left(w_{i}, v_{j}\right)=0$ for all $i, j=1, \ldots, m$. This proves (i).

In the same way, one shows $L\left(w_{i}, w_{j}\right)=0$ for all $w_{i}, w_{j} \in W_{x_{0}}^{+}$, proving (ii).

(iii) Similar calculations and Lemma 9.12(iii) imply that $L_{x_{0}}\left(v_{i}, v_{j}\right)=0$ for all $v_{i}, v_{j} \in W_{x_{0}}^{-}$with $i \neq j$. When $i=j$, one has

$$
L_{x_{0}}\left(v_{i}, v_{i}\right)=\operatorname{Ad}_{x_{0}}\left[X_{-\lambda}^{i}, i \tau_{z} X_{-\lambda}^{i}\right]=\operatorname{Ad}_{x_{0}} i\left[Z_{-\beta_{i}}, \sigma Z_{-\beta_{i}}\right]=b_{i} F_{-2 \lambda}^{0} \quad \text { for } b_{i} \in \mathbb{R} .
$$

In order to prove that $b_{i} \geq 0$ observe that, by Lemma 9.11(iii), one can write $X_{-\lambda}^{i}=X_{\alpha}^{i}+X_{-\alpha}^{i}$ for appropriate $X_{\alpha}^{i} \in \mathfrak{g}^{\alpha}$ and $X_{-\alpha}^{i} \in \mathfrak{g}^{-\alpha}$. Since $z=\exp i A K^{\mathbb{C}}$, with $A \in \mathfrak{a}$ and $\alpha(A)=\pi / 4$, one also has $i \tau_{z} X_{-\lambda}^{i}=\theta X_{\alpha}^{i}-\theta X_{-\alpha}^{i}$ and

$$
\left[X_{-\lambda}^{i}, i \tau_{z} X_{-\lambda}^{i}\right]=\left(\left[X_{\alpha}^{i}, \theta X_{\alpha}^{i}\right]-\left[X_{-\alpha}^{i}, \theta X_{-\alpha}^{i}\right]\right)-\left(\left[X_{\alpha}^{i}, \theta X_{-\alpha}^{i}\right]+\left[X_{-\alpha}^{i}, \theta X_{\alpha}^{i}\right]\right),
$$

which lies in $\mathfrak{a} \oplus Z_{\mathfrak{k}}(\mathfrak{a})$. By [Geatti 2002, Lemma 5.1(i)], the first two terms of the above sum can be written as

$$
\left[X_{\alpha}^{i}, \theta X_{\alpha}^{i}\right]=B\left(X_{\alpha}^{i}, \theta X_{\alpha}^{i}\right) A_{\alpha} \quad \text { and }\left[\theta X_{-\alpha}^{i}, \theta\left(\theta X_{-\alpha}^{i}\right)\right]=B\left(X_{-\alpha}^{i}, \theta X_{-\alpha}^{i}\right) A_{\alpha},
$$

where $A_{\alpha}$ is an element in $\mathfrak{a}$ satisfying the condition $\alpha\left(A_{\alpha}\right)>0$. By the normalization of the reference points chosen in (9-7), one has $\theta X_{2 \lambda}^{0}=-X_{2 \lambda}^{0}$. Hence $L_{x_{0}}\left(v_{i}, v_{i}\right)=b_{i} \operatorname{Ad}_{x_{0}} \theta X_{2 \lambda}^{0}$ for some real number $b_{i} \geq 0$, as claimed. This concludes the proof of (iii).

(iv) Recall that the symmetric space $G^{\prime} / H^{\prime}$ has a reduced restricted root system and that the Lie algebra $\mathfrak{g}^{\prime}$ is given by (9-6). Then the Levi form on $T_{\mathbb{C}}\left(G^{\prime} \cdot x_{0}\right)_{x_{0}}$ can be computed by the methods of Section 9.1. By (9-4), one has

$$
T_{\mathbb{C}}\left(G^{\prime} \cdot x_{0}\right)_{x_{0}}=\operatorname{Ad}_{x_{0}}\left(\mathfrak{g}_{\mathfrak{q}^{\prime}}^{0}\right)^{\mathbb{C}} \oplus \operatorname{Ad}_{x_{0}}\left(\mathfrak{g}_{\mathfrak{q}^{\prime}}^{2 \lambda}\right)^{\mathbb{C}} \quad \text { and } \quad N\left(G^{\prime} \cdot x_{0}\right)_{x_{0}}=\mathbb{R} \operatorname{Ad}_{x_{0}} \theta X_{-2 \lambda}^{0} .
$$

Let $F_{0} \in \operatorname{Ad}_{x_{0}}\left(\mathfrak{g}_{\mathfrak{q}^{\prime}}^{0}\right)^{\mathbb{C}}$ and $F_{2 \lambda} \in \operatorname{Ad}_{x_{0}}\left(\mathfrak{g}_{\mathfrak{q}^{\prime}}^{2 \lambda}\right)^{\mathbb{C}}$. Then by Lemma 9.6 one has

$$
L_{x_{0}}\left(F_{2 \lambda}, F_{2 \lambda}\right)=L_{x_{0}}\left(F_{0}, F_{2 \lambda}\right)=0 \quad \text { and } \quad L_{x_{0}}\left(F_{0}, F_{0}\right)=n F_{-2 \lambda}^{0} \quad \text { where } n \leq 0 .
$$

The next proposition is a direct consequence of Lemmas 9.12 and 9.14.

Proposition 9.15. Let $S$ be the G-orbit of $x_{0}$. The Levi form of $S$ at $x_{0}$ is indefinite if $\mathfrak{g}=\mathfrak{s p}(n, 1)$ or $\mathfrak{g}=\mathfrak{f}_{4}^{*}$. It is definite if $\mathfrak{g}=\mathfrak{s u}(n, 1)$. 
Proof. By Lemma 9.12 and Lemma 9.14(iii) the Levi form $L_{x_{0}}$ is definite on $W_{x_{0}}^{-}$. If $\mathfrak{g}=\mathfrak{s u}(n, 1)$, then $\operatorname{dim} G^{\prime} / H^{\prime}=1$, and the Levi form is identically zero on $T_{\mathbb{C}}\left(G^{\prime} \cdot x_{0}\right)_{x_{0}}$. As a result, in this case the Levi form $L_{x_{0}}$ is definite.

If $\mathfrak{g}=\mathfrak{s p}(n, 1)$ or $\mathfrak{g}=\mathfrak{f}_{4}^{*}$, then $\operatorname{dim} G^{\prime} / H^{\prime}>2$, and the Levi form $L_{x_{0}}$ on $T_{\mathbb{C}}\left(G^{\prime} \cdot x_{0}\right)_{x_{0}}$ is definite of sign opposite to that on $W_{x_{0}}^{-}$; see Proposition 9.7 and Lemma 9.14. As a result, $L_{x_{0}}$ is indefinite, as claimed.

The Levi form of the G-orbit of $y_{0}$. By the same methods, one can compute the tangent space and the Levi form of the $G$-orbit $S$ of the point $y_{0}=\exp i\left(-X_{2 \lambda}^{0}\right) \cdot z$. The tangent space to $S$ at $y_{0}$ is given by $T S_{y_{0}}=T_{\mathbb{C}} S_{y_{0}} \oplus N S_{y_{0}}$, where

$$
T_{\mathbb{C}} S_{y_{0}}=T_{\mathbb{C}}\left(G^{\prime} \cdot y_{0}\right)_{y_{0}} \oplus W_{y_{0}}^{+} \oplus W_{y_{0}}^{-} \quad \text { and } \quad N S_{y_{0}}=\mathbb{R} \operatorname{Ad}_{y_{0}} \theta X_{2 \lambda}^{0} .
$$

Fix $F_{-2 \lambda}^{0}:=\operatorname{Ad}_{y_{0}} \theta X_{2 \lambda}^{0}$ as a generator of $N S_{y_{0}}$.

Lemma 9.16. The following facts hold.

(i) The decomposition of $T_{\mathbb{C}} S_{y_{0}}$ given in (9-12) is orthogonal with respect to the Levi form.

(ii) Let $W \in W_{y_{0}}^{+}$. Then $L_{y_{0}}(W, W) \equiv 0$.

(iii) Let $W \in W_{y_{0}}^{-}$. Then $L_{y_{0}}(W, W)=b F_{-2 \lambda}^{0}$, with $b \geq 0$.

(iv) Let $Z \in T_{\mathbb{C}}\left(G^{\prime} \cdot y_{0}\right)_{y_{0}}$. Then $L_{y_{0}}(Z, Z)=p F_{-2 \lambda}^{0}$, with $p \geq 0$.

Proof. The proof is like the proof of Lemma 9.14. One can check that the Levi form is not identically zero on $W_{y_{0}}^{-}$and has the same signature as on $W_{x_{0}}^{-}$. Part (iv) follows from Lemma 9.8.

Proposition 9.17. Let $S$ be the G-orbit of $y_{0}$. The Levi form of $S$ at $y_{0}$ is definite.

Proof. The proposition follows from Lemma 9.16 and the fact that the Levi form $L_{y_{0}}$ on $W_{y_{0}}^{-}$is not identically zero.

Remark 9.18. If the restricted root system $\Delta_{\mathfrak{b}}$ is nonreduced, then Proposition 9.17 says that the Levi form of the orbits represented by $w_{1}$ and $w_{4}$ in diagrams (4-9) and (4-10) is definite. This is consistent with the fact that these orbits lie in the boundary of the Stein domain $D_{1}(0)$; see Theorem 6.1. When $\mathfrak{g}=\mathfrak{s u}(n, 1)$, by Proposition 9.15, the same is true for the Levi form of the orbits represented by $w_{2}$ and $w_{3}$ in diagram (4-9). We refer to Example 6.3 for a classification of the $G$-invariant Stein domains in $G^{\mathbb{C}} / K^{\mathbb{C}}$ bounded by these orbits. Proposition 9.15 also says that the Levi form of the orbit represented by $w_{2}$ in diagram (4-10) is indefinite. Hence this orbit cannot lie in the boundary of a Stein $G$-invariant domain in $G^{\mathbb{C}} / K^{\mathbb{C}}$. 


\section{Acknowledgments}

We wish to thank Karl Oeljeklaus for pointing out to us Example 8.3 and Stefano Trapani for suggesting the argument in Lemma 7.4.

\section{References}

[Akhiezer and Gindikin 1990] D. N. Akhiezer and S. G. Gindikin, "On Stein extensions of real symmetric spaces”, Math. Ann. 286 (1990), 1-12. MR 91a:32047 Zbl 0681.32022

[Boggess 1991] A. Boggess, CR manifolds and the tangential Cauchy-Riemann complex, CRC Press, Boca Raton, FL, 1991. MR 94e:32035 Zbl 0760.32001

[Burns et al. 2003] D. Burns, S. Halverscheid, and R. Hind, "The geometry of Grauert tubes and complexification of symmetric spaces”, Duke Math. J. 118:3 (2003), 465-491. MR 2004g:32025 Zbl 1044.53039

[Casadio Tarabusi et al. 2000] E. Casadio Tarabusi, A. Iannuzzi, and S. Trapani, "Globalizations, fiber bundles, and envelopes of holomorphy”, Math. Z. 233:3 (2000), 535-551. MR 2001f:32035 Zbl 0961.32021

[Cœuré and Loeb 1986] G. Cœuré and J.-J. Loeb, "Univalence de certaines enveloppes d'holomorphie”, C. R. Acad. Sci. Paris Sér. I Math. 302:2 (1986), 59-61. MR 87k:32024 Zbl 0588.32014

[Fels et al. 2006] G. Fels, A. Huckleberry, and J. A. Wolf, Cycle spaces of flag domains: A complex geometric viewpoint, Progress in Mathematics 245, Birkhäuser, Boston, 2006. MR 2006h:32018 Zbl 1084.22011

[Geatti 2002] L. Geatti, "Invariant domains in the complexification of a noncompact Riemannian symmetric space”, J. Algebra 251:2 (2002), 619-685. MR 2003i:22010 Zbl 1018.32030

[Geatti 2006] L. Geatti, "A remark on the orbit structure of complexified symmetric spaces", preprint, 2006.

[Gindikin and Krötz 2002] S. Gindikin and B. Krötz, "Invariant Stein domains in Stein symmetric spaces and a nonlinear complex convexity theorem", Int. Math. Res. Not. 18 (2002), 959-971. MR 2003d:32026 Zbl 1011.32018

[Grauert 1958] H. Grauert, “Analytische Faserungen über holomorph-vollständigen Räumen”, Math. Ann. 135 (1958), 263-273. MR 20 \#4661 Zbl 0081.07401

[Heinzner 1991] P. Heinzner, “Geometric invariant theory on Stein spaces”, Math. Ann. 289 (1991), 631-662. MR 92j:32116 Zbl 0728.32010

[Heinzner 1993] P. Heinzner, "Equivariant holomorphic extensions of real analytic manifolds", Bull. Soc. Math. France 121:3 (1993), 445-463. MR 94i:32050 Zbl 0794.32022

[Heinzner and Iannuzzi 1997] P. Heinzner and A. Iannuzzi, "Integration of local actions on holomorphic fiber spaces”, Nagoya Math. J. 146 (1997), 31-53. MR 98k:32047 Zbl 0879.55006

[Helgason 1978] S. Helgason, Differential geometry, Lie groups, and symmetric spaces, Pure and Applied Mathematics 80, Academic Press, New York, 1978. MR 80k:53081 Zbl 0451.53038

[Hochschild 1965] G. Hochschild, The structure of Lie groups, Holden-Day, San Francisco, 1965. MR 34 \#7696 Zbl 0131.02702

[Iannuzzi 1999] A. Iannuzzi, "Characterizations of G-tube domains”, Manuscripta Math. 98 (1999), 425-445. MR 2000e:32034 Zbl 0935.32002

[Knapp 1996] A. W. Knapp, Lie groups beyond an introduction, Progress in Mathematics 140, Birkhäuser, Boston, 1996. MR 98b:22002 Zbl 0862.22006 
[Kobayashi 1998] S. Kobayashi, Hyperbolic complex spaces, Grundlehren der Mathematischen Wissenschaften 318, Springer, Berlin, 1998. MR 99m:32026 Zbl 0917.32019

[Krötz and Stanton 2005] B. Krötz and R. J. Stanton, "Holomorphic extensions of representations, II: Geometry and harmonic analysis", Geom. Funct. Anal. 15:1 (2005), 190-245. MR 2006d:43010 Zbl 1078.22009

[Luna 1975] D. Luna, "Sur certaines opérations différentiables des groupes de Lie", Amer. J. Math. 97 (1975), 172-181. MR 51 \#527 Zbl 0334.57022

[Matsuki 1997] T. Matsuki, "Double coset decompositions of reductive Lie groups arising from two involutions”, J. Algebra 197:1 (1997), 49-91. MR 99a:22012 Zbl 0887.22009

[Neeb 1999] K.-H. Neeb, "On the complex geometry of invariant domains in complexified symmetric spaces”, Ann. Inst. Fourier (Grenoble) 49:1 (1999), 177-225. MR 2000i:32040 Zbl 0921.22003

[Palais 1957] R. S. Palais, A global formulation of the Lie theory of transformation groups, Mem. Amer. Math. Soc. 22, American Mathematical Society, Providence, R.I., 1957. MR 22 \#12162 Zbl 0178.26502

[Rossi 1963] H. Rossi, “On envelopes of holomorphy”, Comm. Pure Appl. Math. 16 (1963), 9-17. MR 26 \#6436 Zbl 0113.06001

[Snow 1982] D. M. Snow, "Reductive group actions on Stein spaces", Math. Ann. 259:1 (1982), 79-97. MR 83f:32026 Zbl 0509.32021

[Stein 1956] K. Stein, "Überlagerungen holomorph-vollständiger komplexer Räume", Arch. Math. 7 (1956), 354-361. MR 18,933a Zbl 0072.08002

[Steinberg 1968] R. Steinberg, Endomorphisms of linear algebraic groups, Mem. Amer. Math. Soc. 80, American Mathematical Society, Providence, R.I., 1968. MR 37 \#6288 Zbl 0164.02902

[Wolf 1984] J. A. Wolf, Spaces of constant curvature, 5th ed., Publish or Perish, Houston, TX, 1984. MR 88k:53002

Received April 17, 2008.

LAURA GEATTI

DIPARTIMENTO DI MATEMATICA

UNIVERSITÀ DI ROMA 2 "TOR VERGATA"

VIA DELLA RICERCA SCIENTIFICA

00133 ROMA

ITALY

geatti@mat.uniroma2.it

\author{
ANDREA IANNUZZI \\ DipARTIMENTO Di MATEMATICA \\ UNIVERSITÀ DI ROMA 2 "TOR VERGATA" \\ VIA DELLA RICERCA SCIENTIFICA \\ 00133 ROMA \\ ITALY \\ iannuzzi@mat.uniroma2.it
}

